\title{
BOREL CLASSES AND CLOSED GAMES: WADGE-TYPE AND HUREWICZ-TYPE RESULTS
}

\author{
A. LOUVEAU AND J. SAINT RAYMOND
}

\begin{abstract}
For each countable ordinal $\xi$ and pair $\left(A_{0}, A_{1}\right)$ of disjoint analytic subsets of $2^{\omega}$, we define a closed game $J_{\xi}\left(A_{0}, A_{1}\right)$ and a complete $\Pi_{\xi}^{0}$ subset $H_{\xi}$ of $2^{\omega}$ such that (i) a winning strategy for player I constructs a $\Sigma_{\xi}^{0}$ set separating $A_{0}$ from $A_{1}$; and (ii) a winning strategy for player II constructs a continuous map $\varphi$ : $2^{\omega} \rightarrow A_{0} \cup A_{1}$ with $\varphi^{-1}\left(A_{0}\right)=H_{\xi}$. Applications of this construction include: A proof in second order arithmetics of the statement "every $\Pi_{\xi}^{0}$ non $\Sigma_{\xi}^{0}$ set is $\Pi_{\xi}^{0}$-complete"; an extension to all levels of a theorem of Hurewicz about $\boldsymbol{\Sigma}_{2}^{\delta}$ sets; a new proof of results of Kunugui, Novikov, Bourgain and the authors on Borel sets with sections of given class; extensions of results of Stern and Kechris. Our results are valid in arbitrary Polish spaces, and for the classes in Lavrentieff's and Wadge's hierarchies.
\end{abstract}

Introduction. Let $E=2^{\omega}$ be the usual Cantor space, and following the standard modern terminology of Addison, let $\Sigma_{\xi}^{0}$ and $\Pi_{\xi}^{0}$ denote, respectively, the Borel subsets of $E$ of additive and multiplicative Borel class $\xi$, starting with $\Sigma_{1}^{0}=$ Open and $\Pi_{1}^{0}=$ Closed.

The aim of this paper is to characterize, given a set $A \subset 2^{\omega}$ and one of the Borel classes, say $\Gamma$, whether $A$ is in $\Gamma$ or not. By a "characterization", we have in mind the finding of some mathematical object associated with $A$ and which positively witnesses that $A \in \Gamma$ or that $A \notin \Gamma$.

Let $\Gamma$ be the class $\Sigma_{\xi}^{0}$. If the set $A$ is in $\Sigma_{\xi}^{0}$, a natural candidate for such a witness is simply one particular construction of $A$ as a $\Sigma_{\xi}^{0}$ set (a list of sequences of open sets together with instructions on the operations to be performed to get $A$ in $\xi$ steps). The problem of how to find such constructions, which is deeply connected to the structural properties of sets in the plane with $\Sigma_{\xi}^{0}$ sections, has been extensively investigated in papers by Novikov [N] and Kunugui [Kun] for $\xi=1$ (see also Dellacherie [De]), Saint Raymond [SR 1] for $\xi=2$, Bourgain [B 1, B 2] and Louveau [Lo 1] for $\xi=3$, Louveau [Lo 2] for $\xi \geqslant 3$, in case $A$ is known to be Borel. In case $A$ ranges over larger classes, the problem is studied in papers by Stern [St], Kechris [Ke] and Louveau [Lo 3].

Received by the editors December 26, 1985 and, in revised form, November 19, 1986. These results have been presented at the Annual Meeting of the American Mathematical Society, New Orleans, January 1986 , in a special session on Determinacy and large cardinals.

1980 Mathematics Subject Classification (1985 Revision). Primary 02K30, 04A15, 28A05; Secondary 54H05, 26A21.

Key words and phrases. Borel classes, closed games, Wadge games, determinacy, Hurewicz' theorem. 
Another possible witness for ensuring $A \in \Sigma_{\xi}^{0}$ is given by the notion of reducing map, due to Wadge [W 1]. Suppose we fix some $\Sigma_{\xi}^{0}$ set $A_{0} \subseteq 2^{\omega}$. If $f: 2^{\omega} \rightarrow 2^{\omega}$ is a continuous map which reduces $A$ to $A_{0}$, i.e. satisfies $f^{-1}\left(A_{0}\right)=A$, clearly $A$ is in $\Sigma_{\xi}^{0}$ too, and the reducing map $f$ can be considered a witness of this fact. Note that any $\Sigma_{\xi}^{0}$-construction of $A_{0}$ is transferred via $f$ in a $\Sigma_{\xi}^{0}$-construction of $A$ (by taking counterimages) so that characterization by reducing maps is stronger than by $\Sigma_{\xi}^{0}$-constructions. Note also that reducing maps can be used both ways: If $B_{0} \subseteq 2^{\omega}$ is some set which is not in $\Sigma_{\xi}^{0}$, and $f$ reduces $B_{0}$ to $A$, then $f$ clearly witnesses that $A$ is not in $\Sigma_{\xi}^{0}$. And part of Wadge's work [W 2] consists in showing that for each countable ordinal $\xi$, there is a $\Sigma_{\xi}^{0}$ but not $\Pi_{\xi}^{0}$ set $A_{0} \subseteq 2^{\omega}$, such that if $A$ is $\Sigma_{\xi}^{0}$ there is a map reducing $A$ to $A_{0}$, whereas if $A$ is not $\Sigma_{\xi}^{0}$ there is a map reducing $B_{0}=2^{\omega}-A_{0}$ to $A$ (from this, one gets that any $\Sigma_{\xi}^{0}$ non $\Pi_{\xi}^{0}$ set $A_{0}$ works).

A variant of the preceding notion has been introduced by Hurewicz in his paper [Hu] for the class $\Sigma_{2}^{0}$. Hurewicz proves that if an analytic set $A$ is not $\Sigma_{2}^{0}$, it contains as a closed subset a copy of the Baire space $\omega^{\omega}$. In fact, Hurewicz' construction provides, for $A$ in $\Sigma_{1}^{1}$ but not $\Sigma_{2}^{0}$, a continuous map $f: 2^{\omega} \rightarrow 2^{\omega}$ which reduces the set $\mathbf{P}_{\propto}=\left\{\varepsilon \in 2^{\omega}:\{n: \varepsilon(n)=1\}\right.$ is infinite $\}$ to $A$ and is one-to-one (so that the image $f\left(\mathbf{P}_{x}\right)$ is the desired closed-in- $A$ copy of $\left.\omega^{\omega}\right)$. This result has been extended to other classes in papers by Saint Raymond [SR 2], Van Mill and Van Engelen [vE-vM], and to all Borel classes by Steel [S], where the question of the existence of homeomorphisms between Borel sets is also discussed (see also [vE 1, vE 2]).

Why does one look at such characterizations? The idea behind the investigations is that the actual proof of existence of witnesses should provide strong information on the complexity of the map associating with $A$ its Borel class, and on the uniformity of constructions of varying Borel sets. A parallel can be made with a somewhat simpler situation: A well-known basic fact in the theory of analytic sets (Suslin, see [Lu]) asserts that an uncountable analytic set contains a perfect subset, and thus a copy of the space $2^{\omega}$. Paraphrazing the above discussion, a witness of the countability of $A$ is simply an enumeration in a sequence of its members, whereas a witness for uncountability of $A$ is some continuous one-to-one mapping $f: 2^{\omega} \rightarrow A$. Now the actual proof of the above result was used by Luzin [Lu] to prove another basic fact in the theory, namely that a Borel set in the plane with countable sections is a countable union of Borel graphs (this means that the enumeration witnessing countability of sections can be found in a Borel way). A similar technique was used by Saint Raymond in [SR 1] for proving that Borel sets in the plane with $\Sigma_{2}^{0}$ sections are countable unions of Borel sets with closed sections. He uses the proof of Hurewicz' theorem to show that a $\Sigma_{2}^{0}$-construction of the sections can be found in a Borel way. Closed infinite games of perfect information enter the picture at this point, to give a mathematically precise content to these techniques. In [Da], $\mathrm{M}$. Davis associates with a given analytic set $A \subseteq 2^{\omega}$ a closed game (for player II) such that a winning strategy for player I provides an enumeration of $A$, whereas a winning strategy for player II provides a one-to-one continuous map $f: 2^{\omega} \rightarrow A$. As by a fundamental result of Gale and Stewart closed games are determined, i.e. winning strategies for I or II always exist, Davis' game gives the characterization of 
countability. Moreover, by general results on closed games, winning strategies for player I can be found in a Borel way (when they do exist), and the results of Suslin and Luzin easily follow.

The main goal of this paper is to prove the existence of closed games with properties similar to the games of Davis, but which characterize the Borel classes $\Sigma_{\xi}^{0}$ and $\Pi_{\xi}^{0}$ (Theorem 1.1). By the same arguments as above, the existence of such games "automatically" reproves most of the results in the papers quoted before. We further obtain strong improvements, e.g. (see 5.6).

THEOREM. If $\xi \geqslant 2$ is a countable ordinal, there exists a $\Pi_{\xi}^{0}$ set $H_{\xi} \subseteq 2^{\omega}$ (a Hurewicz test) such that for any Polish space $F$ and pair $A_{0}, A_{1}$ of disjoint analytic sets in $F$, either $A_{0}$ can be separated from $A_{1}$ by a $\Sigma_{\xi}^{0}$ subset of $F$, or there is a continuous one-to-one map $\varphi: 2^{\omega} \rightarrow F$ with $\varphi\left(H_{\xi}\right) \subseteq A_{0}$ and $\varphi\left(2^{\omega}-H_{\xi}\right) \subseteq A_{1}$. Moreover, if $\xi \geqslant 3$, any $\Pi_{\xi}^{0}$ non $\Sigma_{\xi}^{0}$ set $H_{\xi}$ does work.

Our games have predecessors: In his work, Wadge [W 2] introduces a game in order to show the existence of reducing maps proving or disproving that $A$ is in $\Sigma_{\xi}^{0}$. Winning strategies in Wadge's games do provide the reducing maps (see $\$ 1$ ). Wadge's games are Borel (for Borel $A$ ), hence are determined by Martin's fundamental theorem [Ma]. Unfortunately, Martin's theorem is not elementary, by a result of $\mathbf{H}$. Friedman [F] (it roughly needs the existence of uncountably many cardinals), and the information it gives on the complexity of the reducing maps is much too weak to, say, study Borel sets in the plane. By analogy with results of Harrington $[\mathbf{H a}]$ and Steel $[\mathbf{S}]$ asserting that the determinacy of the Wadge games for analytic sets is as complicated as the determinacy of all analytic games, which corresponds to a large cardinal assumption, Steel conjectures in $[\mathbf{S}]$ a similar phenomenon should be true at the Borel level, namely that the existence of reducing maps should imply Borel determinacy. We disprove this conjecture by showing, using our games, that if $A_{0}$ is $\Sigma_{\xi}^{0}$ non $\Pi_{\xi}^{0}$ and $A$ is Borel, Wadge's game for reducing $A$ to $A_{0}$ or $2^{\omega}-A_{0}$ to $A$ is determined, provably in the weak system of second order arithmetics (for finite $\xi$, a similar result has been announced by John $[\mathbf{J}]$ ). Our result also gives a sharp bound on the complexity of the reducing maps (roughly, the complexity of finding a point in an analytic set).

The paper is organized as follows: In the first section, we discuss the notion of Wadge game, state our main existence result and show how it can be used to produce winning strategies in Wadge games. In the second section, we define concrete examples of our closed games, which solve the problem for $\Sigma_{\xi}^{0}$ classes, $\xi=1,2,3$, and for Hausdorff classes of differences of closed sets. The third section is devoted to the main abstract notion of "ramification" of a closed game, which allows by an iterative construction (somewhat close to Martin's iterative construction for proving Borel determinacy) to define inductively on $\xi$ the closed games relevant to the $\Sigma_{\xi}^{0}$ case. The existence theorem is proved in $\S 4$, together with the natural generalization to the case of arbitrary Polish spaces in place of $2^{\omega}$. The remaining sections discuss applications of the results: $\$ 5$ to Hurewicz-type results, $\$ 6$ to the study of Borel sets in the plane and the computation of complexities of the 
$\Sigma_{\xi}^{0}$-witnesses, and $\S 7$ to similar characterizations of the Borel classes, when $A$ is not assumed to be Borel, using stronger set-theoretic assumptions.

Part of our results have been announced in [L-SR].

1. Wadge games. Throughout this paper, we follow the standard modern notations and terminology, with a few explicit exceptions, as it can be found in Moschovakis' basic book [Mo]. E.g., $\omega$ denotes the set of integers, $2^{\omega}=\{\alpha: \omega \rightarrow 2=\{0,1\}\}$ the Cantor space, $\omega^{\omega}=\{\alpha: \omega \rightarrow \omega\}$ the Baire space, $\omega^{<\omega}=\cup_{n} \omega^{n}$ the set of finite sequences from $\omega$, and so on. Letters $n, m, \ldots$ range over $\omega, \varepsilon, \alpha, \beta, \gamma, \ldots$ over $2^{\omega}$ or $\omega^{\omega}, s, t, u$ over $\omega^{<\omega}$ or $2^{<\omega}, \xi, \eta, \theta, \lambda, \ldots$ over ordinals, $E, F$ over (usually Polish) topological spaces, $A, B, C$ over subsets of such spaces. We also refer the reader to Moschovakis' book for unexplained basic notions of descriptive set theory and set theory (especially in the last two sections), and on the fundamentals of infinite games with perfect information.

In the early 1970s, W. Wadge [W 1] has introduced a game to the effect of comparing the descriptive complexity of two sets $A$ and $B$. We discuss this game in the space $2^{\omega}$. For $A, B$ given subsets of $2^{\omega}$, Wadge's game $G_{w}(A, B)$ is defined as follows: Two players, I and II, alternately choose 0's and 1's as follows, player I choosing first:

$$
\begin{array}{lll}
\text { I } & \alpha(0) & \alpha(1) \\
\text { II } & \beta_{0} & \beta(1)
\end{array}
$$

Player I produces $\alpha \in 2^{\omega}$, player II produces $\beta \in 2^{\omega}$, and player II wins the run iff $\alpha \in A \leftrightarrow \beta \in B$.

A winning strategy for player II in this game clearly gives a continuous (in fact Lipschitz) map $f: 2^{\omega} \rightarrow 2^{\omega}$ with $f^{-1}(B)=A$. Similarly, denoting by $\breve{B}$ the complement of $B$ in $2^{\omega}$, a winning strategy for player I defines a Lipschitz function $f$ : $2^{\omega} \rightarrow 2^{\omega}$ with $f^{-1}(A)=\breve{B}$. Define the (pre)-ordering $A \leqslant, B$ iff II has a winning strategy in $G_{w}(A, B)$. (The notation $\leqslant$, comes from [vW], to which we refer the reader for more information on Wadge games.) Now notice that in case $A$ and $B$ are Borel in $2^{\omega}$, the game $G_{w}(A, B)$ is Borel too, hence determined by Martin's theorem [Ma]. Thus one gets

Theorem (W Adge [W 2]). For any two Borel subsets $A, B$ of $2^{\omega}$, either $A \leqslant, B$ or $\check{B} \leqslant, A$.

In other words, if one defines $W(A)=\left\{B \subset 2^{\omega}: B \leqslant, A\right.$ or $\left.\check{B} \leqslant, A\right\}$, then inclusion linearly orders the $W(A)$ 's, for Borel $A$. In fact by a subsequent analysis done by Wadge [W 2] of the $W(A)$ 's, or by some general argument due to Martin (see [vW]), one can show that inclusion wellorders the $W(A)$ 's, $A$ Borel in $2^{\omega}$.

As we said in the introduction, Wadge's proof has some defects: although the statements are formalizable in second order arithmetics, the proof of Martin's theorem needs uncountably many cardinals. From a mathematical standpoint, this means that the (necessarily very complicated) winning stategies given by the proof of Martin do not shed light on the complexity of reducing maps. There is a third 
defect: It is a common feature, when discussing structural properties of Borel sets that there is an underlying property of pairs of analytic sets. In our particular case, let $A$ be Borel in $2^{\omega}$, let $B_{0}, B_{1}$ be two disjoint $\Sigma_{1}^{1}$ (= analytic) subsets of $2^{\omega}$, and consider the following extended Wadge game $G_{w}^{*}\left(A ; B_{0}, B_{1}\right)$ : players I and II play as before, producing respectively $\alpha$ and $\beta$ in $2^{\omega}$, and player II wins if $\alpha \in A \rightarrow \beta \in$ $B_{0}$ and $\alpha \notin A \rightarrow \beta \in B_{1}$ (so $G_{w}(A, B)$ corresponds to $B=B_{0}=\check{B}_{1}$ ). In this extended game, a winning strategy for II gives a Lipschitz map $f: 2^{\omega} \rightarrow 2^{\omega}$ with $f\left(2^{\omega}\right) \subseteq B_{0} \cup B_{1}$ and $f^{-1}\left(B_{0}\right)=A$. And a winning strategy for player I gives a continuous $f: 2^{\omega} \rightarrow 2^{\omega}$ with $f\left(B_{0}\right) \subseteq \check{A}$ and $f\left(B_{1}\right) \subseteq A$, so that the set $C=f^{-1}(\check{A})$ separates $B_{0}$ from $B_{1}$.

These extended games are clearly $\Sigma_{1}^{1}$ (for player II), hence their determinacy seems to require a large cardinal assumption, the existence of sharps. We shall prove that (at least for $A$ in $\Sigma_{\xi}^{0} \backslash \Pi_{\xi}^{0}$ ) the determinacy of these games is provable in the usual framework of set theory-in fact in second order arithmetics.

The method we are going to develop is to replace the above games by closed ones. The usual way to perform this is by "unfolding" the games, i.e. by asking the players to play in the original games, and at the same time to provide witnesses that they are winning the play. Our method will be similar, but a bit more complicated: First we shall fix $A$, and define an unfolding of Wadge games which depends heavily on the (expected) Borel class of $A$. Secondly the unfolding will be only one-sided (on player Il's side). And thirdly (in order to get the game closed) the way witnesses will be played by II will not be continuous, and again depend on the Borel class of $A$.

This means in particular that for defining our games for all Borel sets $A$, one has to go through the complete analysis of Wadge's described classes of Borel sets (see Wadge [W 2], or [Lo 4] for a short account). Although the technique is always the same, details are rather tedious, and we plan to give them in a forthcoming paper. Here we shall focus on the classes which are the most useful ones, the so-called Baire classes $\Sigma_{\xi}^{0}, \Pi_{\xi}^{0}$, and the Lavrentieff classes $D_{\eta}\left(\Sigma_{\xi}^{0}\right)$ of differences of $\Sigma_{\xi}^{0}$ sets, which allow the analysis of the ambiguous Baire class $\Delta_{\xi+1}^{0}$, by a theorem of Hausdorff and Kuratowski (see [Ku]). (For a definition of the $D_{\eta}\left(\Sigma_{\xi}^{0}\right)$ 's, see $\$ 2$.)

For $\Gamma$ one of these classes, let $\check{\Gamma}=\left\{A \subseteq 2^{\omega}: \check{A} \in \Gamma\right\}$ be its dual class. If $H \in \Gamma$, we say $H$ is strategically complete (in $\Gamma$ ) if for any other set $A$ in $\Gamma$, player II wins Wadge's game $G_{w}(A, H)$, i.e. $A \leqslant 1 H$. If $A, B$ are disjoint sets, we say that a set $C$ separates $A$ from $B$ if $A \subseteq C$ and $B \cap C=\varnothing$, and that a class $\Gamma$ separates $(A, B)$ if for some $C \in \Gamma, C$ separates $A$ from $B$.

The main result we obtain in this paper is the following

TheOREM 1. Let $\xi, \eta$ be countable ordinals $(\geqslant 1)$, and $\Gamma$ one of the classes $\Sigma_{\xi}^{0}$, $D_{\eta}\left(\Sigma_{\xi}^{0}\right)$, or their dual classes. There exists a strategically complete set $H_{\Gamma}$ in $\Gamma$, $H_{\Gamma} \subseteq 2^{\omega}$, and for each pair $\left(A_{0}, A_{1}\right)$ of disjoint $\Sigma_{1}^{1}$ subsets of $2^{\omega}$ a closed (for II) game $J_{\Gamma}\left(A_{0}, A_{1}\right)$ such that

(i) A winning strategy for player II in $J_{\Gamma}\left(A_{0}, A_{1}\right)$ induces a winning strategy for the same player in the extended Wadge game $G_{w}^{*}\left(H_{\Gamma} ; A_{0}, A_{1}\right)$.

(ii) A winning strategy for player $\mathrm{I}$ in $J_{\Gamma}\left(A_{0}, A_{1}\right)$ constructs a set $C \subseteq 2^{\omega}$ in the dual class $\check{\Gamma}$ which separates $A_{0}$ from $A_{1}$. 
The next three sections will be devoted to the proof of this theorem. Notice that the separating set $C$ in part (ii) of the above theorem is not given as the counterimage of $\check{H}_{\Gamma}$ via some reducing map (as we said in the Introduction, constructions are weaker than reducing maps - on the other hand, the complexity of the construction will be weaker too, so that it is worth having it). However, one can still recover the instances of Wadge's theorem corresponding to our classes $\Gamma$. Let us do it for $\Gamma=\Pi_{\xi}^{0}$, the other cases being similar. Say that $A \subseteq 2^{\omega}$ is a true $\Pi_{\xi}^{0}$ set if $A$ is $\Pi_{\xi}^{0}$ but not $\Sigma_{\xi}^{0}$.

Corollary 2. Let $A$ be some true $\Pi_{\xi}^{0}$ subset of $2^{\omega}, A_{0}$ and $A_{1}$ two disjoint $\Sigma_{1}^{1}$ subsets of $2^{\omega}$. The extended Wadge game $G_{n}^{*}\left(A ; A_{0}, A_{1}\right)$ is determined. Thus in particular for all Borel sets $B \subseteq 2^{\omega}$,Wadge's game $G_{w}(A, B)$ is determined.

Proof. We prove more precisely that if some $\Sigma_{\xi}^{0}$ set separates $A_{0}$ from $A_{1}$, I has a winning strategy in $G_{*}^{*}\left(A ; A_{0}, A_{1}\right)$, whereas if no such separating set exists, II has a winning strategy. Let $H_{\xi}$ be the strategically complete $\Pi_{\xi}^{0}$ set of Theorem 1. As $A \in \Pi_{\xi}^{0}$, II wins $G_{n^{\prime}}\left(A, H_{\xi}\right)$. Let $J_{\xi}=J_{\Pi_{\xi}^{0}}$. Suppose first no $\Sigma_{\xi}^{0}$ separating set exists. By Theorem 1(ii), I loses $J_{\xi}\left(A_{0}, A_{1}\right)$, hence II wins it and by (i) also wins $G_{u^{*}}^{*}\left(H_{\xi} ; A_{0}, A_{1}\right)$. Composing the winning strategies, II wins $G_{w}^{*}\left(A ; A_{0}, A_{1}\right)$.

Suppose now $C$ is a $\Sigma_{\xi}^{0}$ set separating $A_{0}$ from $A_{1}$. As $\check{C}$ is $\Pi_{\xi}^{0}$, II wins $G_{w}\left(\check{C}, H_{\xi}\right)$. Define, for $i=0$ or $1, A^{(i)}=\left\{\alpha \in 2^{\omega} \mid i^{\wedge} \alpha \in A\right\}$. As $A$ is not $\Sigma_{\xi}^{0}$, at least one of $A^{(0)}$ or $A^{(1)}$, say $A^{\left(i_{0}\right)}$, is not $\sum_{\xi}^{0}$. Applying Theorem 1 to the pair $\left(A^{\left(i_{0}\right)}, \check{A}^{\left(i_{0}\right)}\right)$, we get that II wins the game $J_{\xi}\left(A^{\left(i_{0}\right)}, \check{A}^{\left(i_{0}\right)}\right)$ hence also $G_{w}^{*}\left(H_{\xi} ; A^{\left(i_{0}\right)}, \check{A}^{\left(i_{0}\right)}\right)$. Again by composition, II wins $G_{w}^{*}\left(\check{C} ; A^{\left(i_{0}\right)}, \check{A}^{\left(i_{0}\right)}\right)$. Finally one easily checks that player I wins $G_{*}^{*}\left(A ; A_{0}, A_{1}\right)$ by first playing $i_{0}$, and then following II's winning strategy in $G_{u^{*}}^{*}\left(\check{C} ; A^{\left(i_{0}\right)}, \check{A}^{\left(i_{0}\right)}\right)$.

Notice that this corollary implies in particular a posteriori that any true $\Pi_{\xi}^{0}$ set is strategically complete. Nevertheless, a good part of the proof of Theorem 1 will consist in exhibiting special a priori strategically complete $\Pi_{\xi}^{0}$ sets. We will leave to the reader the checking that our proof of Theorem 1 , hence of the corollary, can be formalized in second order arithmetics.

Notice finally that the proof of the corollary from the theorem is perfectly general, and works for all described Wadge classes, once one knows the existence of corresponding $H_{\Gamma}$ 's and $J_{\Gamma}$ 's, hence giving a proof of Wadge's theorem in second order arithmetics. We leave the general case to our forthcoming paper.

\section{Some (relatively) simple games.}

2.1. The cases $\xi=2$ and $\xi=3$. The reader who only wants to see the proof of Theorem 1.1. may skip this subsection, and go to 2.2 where we present the basic games, corresponding to the $\Sigma_{1}^{0}$ and $D_{\eta}\left(\Sigma_{1}^{0}\right)$ cases. But although the games for $\xi=2$ and 3 are just (variants of) particular cases of the general construction, we start with them because (i) the game for $\xi=2$, which we extracted from one of the proofs of Hurewicz' theorem, was the first game we obtained, (ii) the games for $\xi=1$ are a bit too simple, and the general case too abstract, to give the flavor of the arguments, 
and (iii) if our construction is ever to be used again for other purposes, there are some chances that it will be through specific examples.

Let us first consider $\xi=2$, i.e. $\Sigma_{2}^{0}$ and $\Pi_{2}^{0}$ sets. First we define strategically complete sets in these classes: Let $\mathbf{P}_{f}=\left\{\varepsilon \in 2^{\omega} \mid \varepsilon\right.$ is eventually zero $\}$, and $\mathbf{P}_{\infty}=\check{\mathbf{P}}_{f}$. We claim that $\mathbf{P}_{f}$ is a $\Sigma_{2}^{0}$ strategically complete set (so that $\mathbf{P}_{\infty}$ is strategically complete $\Pi_{2}^{0}$ ). To see this, let $A \subseteq 2^{\omega}$ be $\Sigma_{2}^{0}$, and write $A=\cup_{n} F_{n}$, with $F_{n}$ closed in $2^{\omega}$, say $F_{n}=\left[T_{n}\right]$ for some tree $T_{n}$ on $\omega$ (i.e. $F_{n}=\left\{\alpha \in 2^{\omega}: \forall n \alpha \uparrow_{n} \in T_{n}\right\}$ ). Let II play in $G_{w}\left(A, \mathbf{P}_{f}\right)$ as follows: if at stage $n$ I has played $u$ of length $n+1$ and II $v$ of length $n$, let $k=\operatorname{card}(\{i<n: v(i)=1\})$ and let II play 0 if $u \in T_{k}$, and 1 otherwise. This strategy is clearly winning for II.

We now introduce, for $A_{0}, A_{1}$ disjoint $\Sigma_{1}^{1}$ sets in $2^{\omega}$, a closed game $J\left(\mathbf{P}_{\infty} ; A_{0}, A_{1}\right)$ (it is a variant of the game $J_{2}\left(A_{0}, A_{1}\right)$ we shall define in $\left.\S 4\right)$. We choose trees $T_{0}$ and $T_{1}$ in $(2 \times \omega)^{<\omega}$ with $A_{i}=\left\{\alpha \in 2^{\omega}: \exists \beta \in \omega^{\omega} \forall n\left(\alpha \uparrow_{n}, \beta \uparrow_{n}\right) \in T_{i}\right\}$ for $i=0$ or 1 . In the game, players I and II play as follows:

I $\quad \varepsilon(0)$

II $\quad \alpha(0), \beta(0)$

$\alpha(1), \beta(1)$

where $\varepsilon(i), \alpha(i)$ are 0 or 1 , and $\beta(i) \in \omega$, thus producing, respectively, $\varepsilon \in 2^{\omega}$ and $(\alpha, \beta) \in 2^{\omega} \times \omega^{\omega}$. The play is a win for II if all finite positions $\left(\varepsilon \uparrow_{k}, \alpha \uparrow_{k}, \beta \uparrow_{k}\right)$ are legal (so that the game is closed for player II), where by legal we mean here the following: If the last value $\varepsilon(k-1)$ is 0 , let $n$ be least such that $\varepsilon(n)=\varepsilon(n+1)=$ $\cdots=\varepsilon(k-1)=0$, and say the position is legal if $\left(\alpha \uparrow_{k-n},\langle\beta(n), \ldots, \beta(k-1)\rangle\right)$ $\in T_{1}$. In the other case, let $j_{0}<j_{1}<\cdots<j_{l-1}=k-1$ be the increasing enumeration of those $j$ 's for which $\varepsilon(j)=1$, and say the position is legal if $\left(\alpha \uparrow_{l},\left\langle\beta\left(j_{0}\right), \ldots, \beta\left(j_{l-1}\right)\right\rangle\right) \in T_{0}$. This defines the game $J\left(\mathbf{P}_{\infty}, A_{0}, A_{1}\right)$ entirely. We now prove that this game satisfies (i) and (ii) of Theorem 1.1.

First suppose player II has a winning strategy $\tau$ in this game, and let $\tau^{*}$ be the strategy in $G_{w^{\prime}}^{*}\left(\mathbf{P}_{\infty} ; A_{0}, A_{1}\right)$ obtained by deleting the $\beta$ moves of II. If player I plays $\varepsilon \in \mathbf{P}_{f}$, let $n$ be least so that $\varepsilon(i)=0$ for $i \geqslant n$. By $\tau$, II answers $(\alpha, \beta)$ and by the legality of finite positions, $\beta^{*}=\langle\beta(n), \beta(n+1), \ldots\rangle$ satisfies $\left(\alpha, \beta^{*}\right) \in\left[T_{1}\right]$, hence $\alpha=\tau^{*}(\varepsilon)$ is in $A_{1}$. Now if $\varepsilon \in \mathbf{P}_{\infty}$ and $\left(j_{i}\right)_{i \in \omega}$ is the increasing enumeration of the $j$ 's with $\varepsilon(j)=1$, then, by the legality of finite positions, $\beta^{*}(i)=\beta\left(j_{i}\right)$ satisfies $\left(\alpha, \beta^{*}\right) \in\left[T_{0}\right]$, hence $\alpha=\tau^{*}(\varepsilon)$ is in $A_{0}$. This shows $\tau^{*}$ is winning for II in $G_{w}^{*}\left(\mathbf{P}_{\infty} ; A_{0}, A_{1}\right)$. Now suppose $\sigma$ is a winning strategy for player $\mathrm{I}$ in the game. For a fixed $\alpha \in 2^{\omega}$, say $u \in \omega^{n}$ is $(\sigma, \alpha)$-legal if all positions of length $i \leqslant n$ where II plays $\alpha \uparrow_{i}, u \uparrow_{i}$ and I answers by $\sigma$ are legal. Let $C=\left\{\alpha \in 2^{\omega} \mid \exists n \exists u \in \omega^{n}[(n=0\right.$ or $\left.\sigma\left(\alpha \uparrow_{n-1}, u \uparrow_{n-1}\right)=1\right)$ and $(u$ is $(\sigma, \alpha)$-legal) and $\forall v(v$ is $(\sigma, \alpha)$-legal and $v$ extends $u \rightarrow \sigma(\alpha\lceil\operatorname{lh}(1), v)=0)]\} . C$ is clearly in $\Sigma_{2}^{0}$, and we claim it separates $A_{0}$ from $A_{1}$. We argue by contradiction: If $\alpha \in A_{1} \cap C$, we pick $u \in \omega^{n}$ witnessing $\alpha \in C$, and $\beta \in \omega^{\omega}$ so that $(\alpha, \beta) \in\left[T_{1}\right]$. Consider the play where II plays $\left(\alpha, u^{\wedge} \beta\right)$ and I follows $\sigma$. By hypothesis, the positions up to stage $n$ are legal. Now by induction if for $k \geqslant n$ the position at stage $k$ is legal, then by the definition of $C$ the answer by $\sigma$ is 0 , hence calls for extending $\left(\alpha \uparrow_{k-n}, \beta \uparrow_{k-n}\right)$ inside $T_{1}$, and the next position is again legal. So this play defeats $\sigma$, a contradiction which proves 
$C \cap A_{1}=\varnothing$. Similarly, suppose $\alpha \in A_{0}-C$, and let $\beta$ be so that $(\alpha, \beta) \in\left[T_{0}\right]$. As $\alpha \notin C$, there is a function $f$ associating with each $(\sigma, \alpha)$-legal sequence $u$ which is $\varnothing$ or for which $\sigma$ calls for 1 a $(\sigma, \alpha)$-legal extension $f(u)$ of $u$ so that $\sigma\left(\alpha \uparrow_{\operatorname{lh} f(u)}, f(u)\right)$ $=1$, and we can choose $f(u)$ of minimal length with these properties (so that in between $\sigma$ calls for 0 's). Consider then the play where I follows $\sigma$ and II plays $\alpha$ together with $f(\varnothing)^{\wedge} \beta(0)$, then according to $f\left(f\left(\varnothing^{\wedge} \beta(0)\right)^{\wedge} \beta(1)\right)$, etc . . one easily checks by induction that all positions of this play are legal, hence again $\sigma$ is defeated, a contradiction which proves $A_{0} \subseteq C$ and finishes the claim.

The game for $\xi=3$ is similar, although notationally more complicated. Choose first some canonical bijection between $\omega^{2}$ and $\omega$, say $\langle i, j\rangle=\frac{1}{2}(i+j)(i+j+1)+i$, with inverse maps $(n)_{0}$ and $(n)_{1}$. Define a $\Sigma_{3}^{0}$ set $G$ by

$$
G=\left\{\alpha \in 2^{\omega} \mid \text { for some } n\{i: \alpha(\langle n, i\rangle)=1\} \text { is infinite }\right\}
$$

and let $H=\check{G}$. First we claim $G$ is strategically complete: If $A$ is $\Sigma_{3}^{0}$, say $A=\bigcup_{n} A_{n}$ with $A_{n} \in \Pi_{2}^{0}$, let $\sigma_{n}$ be winning for player II in $G_{w}\left(A_{n}, \mathbf{P}_{\infty}\right)$ and let II play in $G_{w}(A, G)$ as answer to $u$ of length $n+1$ the answer by $\sigma_{(n)_{0}}$ to $u \uparrow_{(n)_{1}+1}$. This is easily winning for II in $G_{w}(A, G)$.

For $A_{0}, A_{1}$ with associated trees $T_{0}$ and $T_{1}$ as before, we now define the closed game $J\left(H, A_{0}, A_{1}\right)$. The basic moves are as before, and again II wins if all finite positions $\left(\varepsilon \uparrow_{k}, \alpha \uparrow_{k}, \beta \uparrow_{k}\right)$ are legal, so that we only need to define the new notion of legal position in the game. For this, we inductively associate with each position $t_{k}=\left(\varepsilon \uparrow_{k}, \alpha \uparrow_{k}, \beta \uparrow_{k}\right)$ finite sequences of integers $s^{0}\left(t_{k}\right)$ and $s_{n}^{1}\left(t_{k}\right), n \in \omega$, starting with $s^{0}(\varnothing)=s_{n}^{1}(\varnothing)=\varnothing$, as follows: Suppose it has been done up to $k$. If $\varepsilon(k)=0$, we set $s^{0}\left(t_{k+1}\right)=s^{0}\left(t_{k}\right)^{\wedge} \beta(k)$ and $s_{n}^{1}\left(t_{k+1}\right)=s_{n}^{1}\left(t_{k}\right)$ for all $n$. If now $\varepsilon(k)=1$, with $k=\left\langle k_{0}, k_{1}\right\rangle$, we set $s^{0}\left(t_{k+1}\right)=s^{0}\left(t_{k}\right) \uparrow_{k_{0}} \quad\left(=s^{0}\left(t_{k}\right)\right.$ if $\left.\ln \left(s^{0}\left(t_{k}\right)\right) \leqslant k_{0}\right)$ and we set

$$
s_{n}^{1}\left(t_{k+1}\right)= \begin{cases}s_{n}^{1}\left(t_{k}\right) & \text { for } n<k_{0}, \\ s_{n}^{1}\left(t_{k}\right)^{\wedge} \beta(k) & \text { for } n=k_{0} \\ \varnothing & \text { for } n>k_{0}\end{cases}
$$

Finally we say that $t_{k+1}$ is legal if $\left(\alpha \uparrow_{\operatorname{lh} s^{0}\left(t_{k+1}\right)}, s^{0}\left(t_{k+1}\right)\right) \in T_{0}$ and for all $n$ $\left(\alpha \uparrow_{\mathrm{lh} s_{n}^{1}\left(t_{k+1}\right)}, s_{n}^{1}\left(t_{k+1}\right)\right) \in T_{1}$ (this is a finite condition, as clearly $s_{n}^{1}=\varnothing$ for all but finitely many integers).

Intuitively, at each moment $t$ of the game player II has produced one beginning $s^{0}(t)$ of a witness that eventually his play $\alpha$ will be in $A_{0}$ and various beginnings of witnesses that $\alpha$ will be in $A_{1}$ (the $s_{n}^{1}(t)$ 's). When player I plays 0 , he asks player II to extend his $A_{0}$-witness. When player I plays 1 , he asks II to extend his $n$th $A_{1}$-witness, where $n=(\operatorname{lh} t)_{0}$. But at the same time, he allows II to revise his previous beginnings $s_{m}^{1}(t)$, for $m>n$, and to revise his choice of the $A_{0}$-witness-at least after the first $n$ values of $s^{0}(t)$. 
This defines entirely the game $J\left(H, A_{0}, A_{1}\right)$, and we now proceed to show it satisfies conclusions (i) and (ii) of Theorem 1.1.

First suppose $\tau$ is winning for player II, and let $\tau^{*}$ be the strategy in $G_{w}^{*}\left(H ; A_{0}, A_{1}\right)$ gotten by deleting the $\beta$-moves. Let $\varepsilon$ be played by $\mathrm{I}$, and $t_{k}=$ $\left(\varepsilon \uparrow_{k}, \alpha \uparrow_{k}, \beta \uparrow_{k}\right)$ be the (legal) positions obtained by letting II follow $\tau$. If $\varepsilon \in H$, we want to show $\alpha=\tau^{*}(\varepsilon)$ is in $A_{0}$. We know that for all $n\{i: \varepsilon(\langle n, i\rangle)=1\}$ is finite. In particular, there is a least $n_{0}$ so that $\left(n_{0}\right)_{0}=0$ and for all $n \geqslant n_{0}$ with $(n)_{0}=0, \varepsilon(n)=0$. One checks that as $t_{k}, k>n_{0}$, is legal, then $s^{0}\left(t_{k}\right)$ has length $\geqslant 1$ and $s^{0}\left(t_{k}\right)(0)$ is a constant $\beta^{*}(0)$ independent of $k$. Let then $n_{1}>n_{0}$ be least so that $\left(n_{1}\right)_{0}=1$, and for all $n \geqslant n_{1}$ with $(n)_{0}=1, \varepsilon(n)=0$. Again one checks that, for $k>n_{1}, s^{0}\left(t_{k}\right)$ has length $\geqslant 2$ and $s^{0}\left(t_{k}\right)(1)=\beta^{*}(1)$, etc.

This allows to construct a sequence $\left(n_{i}\right)_{i \in \omega}$ and $\beta^{*}$ so that the sequence $\left(s^{0}\left(t_{k}\right)\right)_{k \in \omega}$ converges to $\beta^{*}$. And as the $t_{k}$ are legal, this implies $\left(\alpha, \beta^{*}\right) \in\left[T_{0}\right]$, hence $\alpha \in A_{0}$. Similarly, suppose now $\varepsilon \in G$, and let $k_{0}$ be least so that $\left\{i: \varepsilon\left(\left\langle k_{0}, i\right\rangle\right)=1\right\}$ is infinite, and $n_{0}$ be least so that $\left(n_{0}\right)_{0}=k_{0}, \varepsilon\left(n_{0}\right)=1$, and for $n \geqslant n_{0}$ with $(n)_{0}<k_{0}, \varepsilon(n)=0$. Now let $\left(n_{i}\right)_{i \in \omega}$ be the increasing enumeration of those $n \geqslant n_{0}$ for which $(n)_{0}=k_{0}$ and $\varepsilon(n)=1$, and let $\beta^{*}(i)=\beta\left(n_{i}\right)$. We claim that the sequence $\left(s_{k_{0}}^{1}\left(t_{k}\right)\right)_{k \in \omega}$ converges to $\beta^{*}$, hence in particular $\left(\alpha, \beta^{*}\right) \in\left[T_{1}\right]$ and $\alpha \in A_{1}$. To see this, note first that $s_{k_{0}}^{1}\left(t_{n_{0}}\right)=\varnothing$. If not, this means that for some $n<n_{0}$ with $(n)_{0}=k_{0}$, one has $\varepsilon(n)=1$, and moreover for all $n^{\prime}, n \leqslant n^{\prime}<n_{0}$ with $\left(n^{\prime}\right)_{0}<k \varepsilon\left(n^{\prime}\right)=0$ (for if not $s_{k_{0}}^{1}\left(t_{n}\right)$ would have been erased by the last clause in the definition of the $s_{n}^{1}$ 's). But this contradicts the minimality of $n_{0}$. So $s_{k_{0}}^{1}\left(t_{n_{0}}\right)=\varnothing$ and $s_{k_{0}}^{1}\left(t_{n_{0}+1}\right)=\beta\left(n_{0}\right)$. Now notice that clause 3 in the definition of the $s_{n}^{1}$ s will never apply to $s_{k_{0}}^{1}\left(t_{n}\right)$ for $n \geqslant n_{0}$, and this easily proves the claim and shows that $\tau^{*}$ is winning in $G_{w}^{*}\left(H ; A_{0}, A_{1}\right)$.

Now suppose player I has some winning strategy $\sigma$ in $J\left(H ; A_{0}, A_{1}\right)$.

For $u$ in $\omega^{<\omega}$ and $\alpha$ in $2^{\omega}$, consider the position $t$ in the game where II has played $u$ and the beginning of $\alpha$ and I followed $\sigma$. We say $u$ is $(\sigma, \alpha)$-legal if this play $t$ is legal and we denote (ambiguously) by $s^{0}(u)$ (resp. $s_{n}^{1}(u)$ ) the corresponding $s^{0}(t)$ (resp. $s_{n}^{1}(t)$ ).

We define a set $C$ by: $\alpha \in C \leftrightarrow \exists m=\langle k, l\rangle \quad \exists u \in \omega^{m}$ such that

(1) $\left(u\right.$ is $(\sigma, \alpha)$-legal and $\sigma\left(\alpha \uparrow_{m}, u\right)=1$ and $\left.s_{k}^{1}(u)=\varnothing\right)$

and (2) $\forall v\left(\left(v\right.\right.$ is $(\sigma, \alpha)$-legal and $v$ extends $u$ and $\left.\left.(\operatorname{lh} v)_{0}<k\right) \rightarrow \sigma\left(\alpha \uparrow_{\operatorname{lh} v}, v\right)=0\right)$

and (3) $\forall v \forall p\left(\left(v^{\wedge} p\right.\right.$ is $(\sigma, \alpha)$-legal and $v$ extends $u$ and $\sigma\left(\alpha \uparrow_{\operatorname{lh} v}, v\right)=1$ and $\left.(\operatorname{lh} v)_{0}=k\right) \rightarrow \exists w\left(w\right.$ is $(\sigma, \alpha)$-legal and $w$ extends $v^{\wedge} p$ and $(\operatorname{lh} w)_{0}=k$ and $\left.\sigma\left(\alpha \prod_{\operatorname{lh} w}, w\right)=1\right)$ )

A moment's reflection shows that $C$ is $\Sigma_{3}^{0}$. We now argue, by contradiction, that $C$ separates $A_{0}$ from $A_{1}$.

Suppose first $\alpha \in A_{1} \cap C$, and pick $\beta$ such that $(\alpha, \beta) \in\left[T_{1}\right]$, and $u$ so that clauses (1), (2), (3) above are satisfied by $\alpha$ and $u$. We now define a play $\left(\alpha, \beta^{*}\right)$ of player II defeating $\sigma$, a contradiction which shows $A_{1} \cap C=\varnothing$. First let II play according to $\alpha$ and $u$, up to stage $m_{0}=\langle k, l\rangle=\operatorname{lh} u$. One checks that the strategy $\sigma$ calls for extending $s_{k}^{1}(u)=\varnothing$, hence that $\beta(0)=\beta^{*}\left(m_{0}\right)$ is a legal play at this stage. Now applying clause (3) to $u$ and $p=\beta(0)$, we get a $(\sigma, \alpha)$-legal extension $w$, 
which we choose ofD minimal length $m_{1}$, such that $\left(m_{1}\right)_{0}=k$ and $\sigma\left(\alpha \uparrow_{m_{1}}, w\right)=1$. By the second clause and minimality of $m_{1}$, the answers by $\sigma$ at stages $n$ with $m_{0}<n<m_{1}$ and $(n)_{0} \leqslant k$ are 0 , hence $s_{k}^{1}(w)=s_{k}^{1}\left(u^{\wedge} \beta(0)\right)=\langle\beta(0)\rangle$ and at stage $m_{1}, \sigma$ calls for extending it, hence $\beta(1)$ is a legal play for II at this stage. Continuing this way, one easily gets some infinite play $\beta^{*}$ so that $\left(\alpha, \beta^{*}\right)$ defeats $\sigma$.

Finally suppose $\alpha \in A_{0} \backslash C$, and let $\beta$ be such that $(\alpha, \beta) \in\left[T_{0}\right]$. We again construct $\beta^{*}$ so that $\left(\alpha, \beta^{*}\right)$ defeats $\sigma$. Let II start playing $\alpha$ and $\beta$ until some stage $m$ is reached with $\sigma\left(\alpha \uparrow_{m}, \beta \uparrow_{m}\right)=1$. Notice that if no such stage exists, $(\alpha, \beta)$ defeats $\sigma$ and we are done. In the other case, pick $u$ with minimal $k_{0}=(\operatorname{lh} u)_{0}$ and then of minimal length $m_{0}$ so that $u$ is $(\sigma, \alpha)$-legal, extends $\beta \uparrow_{m 4}$ and satisfies $\sigma\left(\alpha \uparrow_{m_{0}}, u\right)=1$. As $\alpha \notin C$, one of clauses (1), (2) or (3) must fail for $u$. Now clause (2) is satisfied by the minimality of $k_{0}$, and clause (1) too by the minimality of $m_{0}$. So clause (3) fails and there is some $(\sigma, \alpha)$-legal extension $v$ of $u$ of length $n_{0}$ and some $p$ such that $\left(n_{0}\right)_{0}=k_{0}, \sigma\left(\alpha \uparrow_{n_{0}}, v\right)=1$ and $v^{\wedge} p$ is $(\sigma, \alpha)$-legal, but any $(\sigma, \alpha)$-legal extension $w$ of $v^{\wedge} p$ with $(\mathrm{lh} w)_{0}=k_{0}$ gets answer 0 by $\sigma$. Let II play according to $\alpha$ and $v^{\wedge} p$ up to stage $n_{0}$. Notice now that $k_{0} \leqslant m$, and use the minimality of $k_{0}$ to check that $s^{0}\left(v^{\wedge} p\right)=\beta \uparrow_{k_{0}}$. Hence after stage $n_{0}$, II can legally follow $\alpha$ and $\beta$, until some stage $m^{\prime}$ is reached with $\sigma\left(\alpha \uparrow_{m^{\prime}}, u_{m^{\prime}}\right)=1$-if there is no such stage, we are done. One can then repeat the preceding argument, and so on, and construct the desired $\beta^{*}$. This completes the proof for $\xi=3$.

2.2. The basic games $J_{1}$ and $J_{1, \eta}$. We now present the first step in our inductive construction. These games take care of the classes $\Sigma_{1}^{0}$ and $D_{\eta}\left(\Sigma_{1}^{0}\right)$. Fix once and for all a pair $\left(A_{0}, A_{1}\right)$ of disjoint $\Sigma_{1}^{1}$ sets in $2^{\omega}$, with associated trees $T_{0}, T_{1}$ on $2 \times \omega$, so that for $i=0$ or 1

$$
A_{i}=p\left(\left[T_{i}\right]\right)=\left\{\alpha \in 2^{\omega} \mid \exists \beta \in \omega^{\omega} \forall n\left(\alpha \uparrow_{n}, \beta \uparrow_{n}\right) \in T_{i}\right\} .
$$

Let $\underline{0}: \omega \rightarrow 2$ be the constant function with value 0 , and let

$$
H_{1}=\{\underline{0}\}, \quad G_{1}=\check{H}_{1}=\left\{\varepsilon \in 2^{\omega} \mid \text { for some } n \varepsilon(n)=1\right\} .
$$

One trivially checks that for each closed $A \subseteq 2^{\omega}, A \leqslant, H_{1}$, i.e., $H_{1}$ is a strategically complete $\Pi_{1}^{0}$ set.

We define the game $J_{1}\left(A_{0}, A_{1}\right)$ as follows

$$
\begin{array}{lll}
\text { I } & \varepsilon(0) & \multicolumn{2}{c}{\varepsilon(1)} \\
\text { II } & \alpha(0), \beta(0) & \alpha(1), \beta(1)
\end{array}
$$

At the end of a play, I produces $\varepsilon \in 2^{\omega}$ and II $(\alpha, \beta) \in 2^{\omega} \times \omega^{\omega}$. We say that II wins if all finite positions are legal, where $u_{k}=\left(\varepsilon \uparrow_{k}, \alpha \uparrow_{k}, \beta \uparrow_{k}\right)$ is legal if either for all $i<k \varepsilon(i)=0$ and $\left(\alpha \uparrow_{k}, \beta \uparrow_{k}\right) \in T_{0}$, or for $i_{0}$ the least $i<k$ with $\varepsilon(i)=1$ one has $\left(\alpha \uparrow_{h-i_{0},},\left\langle\beta\left(i_{0}\right), \ldots, \beta(k-1)\right\rangle\right) \in T_{1}$.

The game $J_{1}\left(A_{0}, A_{1}\right)$ is clearly closed. We let $\Sigma=2^{\omega^{<\omega}}=\left\{\sigma: \omega^{<\omega} \rightarrow\{0,1\}\right\}$, where elements of $\Sigma$ are viewed as strategies in games where I produces $\varepsilon \in 2^{\omega}$ and II produces $\beta \in \omega^{\omega}$. For $\alpha \in 2^{\omega}$ and $\sigma \in \Sigma$, say that a sequence $u \in \omega^{n}$ is $(\sigma, \alpha)$-legal if all positions of length $\leqslant n$ in $J_{1}\left(A_{0}, A_{1}\right)$ where II follows $\alpha$ and $u$ and I answers against $u$ by $\sigma$ are legal. 
For $\alpha \in 2^{\omega}$ we denote by $J_{1}\left(A_{0}, A_{1}\right) \uparrow_{\alpha}$ the closed game obtained from $J_{1}\left(A_{0}, A_{1}\right)$ by fixing $\alpha$.

Let $C \subseteq 2^{\omega} \times \Sigma$ be the open set defined by

$$
(\alpha, \sigma) \in C \leftrightarrow \exists u \in \omega^{<\omega}(u \text { is }(\sigma, \alpha) \text {-legal and } \sigma(u)=1) \text {. }
$$

Lemma 1. (i) Let $(\varepsilon, \alpha, \beta)$ be a win for II in $J_{1}\left(A_{0}, A_{1}\right)$. If $\varepsilon=\underline{0}$, then $\alpha \in A_{0}$. And if $\varepsilon \neq 0$, then $\alpha \in A_{1}$.

(ii) Suppose $\sigma$ is winning for player I in $J_{1}\left(A_{0}, A_{1}\right) \uparrow_{\alpha}$ for some $\alpha \in 2^{\omega}$. Then if $\alpha$ is in $A_{0},(\alpha, \sigma) \in C$, and if $\alpha$ is in $A_{1},(\alpha, \sigma) \notin C$.

Proof. (i) is immediate: If $\varepsilon=\underline{0}$, one gets from the definition of legal positions that for all $i\left(\alpha \uparrow_{i}, \beta \uparrow_{i}\right) \in T_{0}$, hence $\alpha \in A_{0}$. Now if $\varepsilon \neq \underline{0}$, and $i_{0}$ is least so that $\varepsilon\left(i_{0}\right)=1$, one gets for all $i\left(\alpha \uparrow_{i},\left\langle\beta\left(i_{0}\right), \ldots, \beta\left(i_{0}+i-1\right)\right\rangle\right) \in T_{1}$ and $\alpha \in A_{1}$.

(ii) We argue by contradiction. Suppose $\sigma$ is winning in $J_{1} \uparrow_{\alpha}$ for I, and $\alpha \in A_{1}$ but $(\alpha, \sigma) \in C$. Choose $u$ of minimal length so that $u$ is $(\sigma, \alpha)$-legal and $\sigma(u)=1$, and $\beta \in \omega^{\omega}$ so that $(\alpha, \beta) \in\left[T_{1}\right]$. Then easily $u^{\wedge} \beta$ is a play in $J_{1} \uparrow_{\alpha}$ defeating $\sigma$, a contradiction. Similarly, if $\alpha \in A_{0}$ but $(\alpha, \sigma) \notin C$, choose $\beta$ so that $(\alpha, \beta) \in\left[T_{0}\right]$ and check that $\beta$ is a play in $J_{1} \uparrow_{\alpha}$ defeating $\sigma$.

COROllary 2. (i) If $\tau$ is a winning strategy for II in $J_{1}\left(A_{0}, A_{1}\right)$, the corresponding strategy $\tau^{*}$ in $G_{w}^{*}\left(H_{1} ; A_{0}, A_{1}\right)$ obtained by deleting the $\beta$ moves is also winning for II.

(ii) If $\sigma$ is a winning strategy for $\mathrm{I}$ in $J_{1}\left(A_{0}, A_{1}\right)$, and $\sigma_{\alpha}$ denotes the element of $\Sigma$ obtained by fixing $\alpha$, then the open set $C_{\sigma}=\left\{\alpha \mid\left(\alpha, \sigma_{\alpha}\right) \in C\right\}$ separates $A_{0}$ from $A_{1}$.

The above corollary is Theorem 1.1. for $\xi=1$. We now turn to the classes $D_{\eta}\left(\Sigma_{1}^{0}\right)$. Let $\eta \geqslant 1$ be some countable ordinal. If $\left(B_{\zeta}\right)_{\zeta<\eta}$ is an increasing sequence of sets indexed by $\eta$, we define the set $D_{\eta}\left(\left(B_{\zeta}\right)\right)=\left\{x \mid \exists \zeta<\eta\left(x \in B_{\zeta}\right)\right.$ and the least such $\zeta$ is odd iff $\eta$ is even $\}$. So in particular $D_{1}\left(\left(B_{0}\right)\right)=B_{0}, D_{2}\left(B_{0}, B_{1}\right)=B_{1}-B_{0}$, $D_{3}\left(B_{0}, B_{1}, B_{2}\right)=B_{0} \cup\left(B_{2}-B_{1}\right)$, etc. If $\Gamma$ is some class (we shall use it for $\Sigma_{\xi}^{0}$ only), we let $D_{\eta}(\Gamma)$ be the class of all $D_{\eta}\left(\left(B_{\zeta}\right)_{\zeta<\eta}\right)$, for increasing sequences $\left(B_{\zeta}\right)$ of sets in $\Gamma$. (Kuratowski [Kur] uses a slightly different notion, working with decreasing sequences and the classes $\left.\Pi_{\xi}^{0}\right)$. The classes $D_{\eta}\left(\Sigma_{\xi}^{0}\right)$ and their dual classes are called the Lavrentieff or "small Baire" classes. A well-known theorem of Hausdorff and Kuratowski (see [Kur]) asserts that in any Polish space, the ambiguous Baire class $\Delta_{\xi+1}^{0}=\Sigma_{\xi+1}^{0} \cap \Pi_{\xi+1}^{0}$ is $\bigcup_{\eta<\omega_{1}} D_{\eta}\left(\Sigma_{\xi}^{0}\right)$.

We now define for each $\eta<\omega_{1}$ a set $G_{1, \eta}$ in $D_{\eta}\left(\Sigma_{1}^{0}\right)$ which is strategically complete for this class. Let $\psi$ be some given bijection: $\eta \times \omega \rightarrow \omega$, with inverse maps $\psi_{0}$ and $\psi_{1}$, and such that for fixed $\zeta<\eta, \psi(\zeta, \cdot): \omega \rightarrow \omega$ is increasing. We associate to each finite sequence $u \in 2^{<\omega}$ an ordinal $\zeta(u) \leqslant \eta$ by

$$
\zeta(u)=\left\{\begin{array}{l}
\min \left\{\psi_{0}(k): k<\operatorname{lh} u \text { and } u(k)=1\right\} \quad \text { if this set is } \neq \varnothing, \\
\eta \quad \text { if the preceding set is empty. }
\end{array}\right.
$$

Clearly if $v$ extends $u, \zeta(v) \leqslant \zeta(u)$, so for each $\varepsilon \in 2^{\omega}$ the sequence $\zeta\left(\varepsilon \uparrow_{k}\right)$ is eventually constant and we call this constant $\zeta(\varepsilon)$. We finally set $G_{1, \eta}=\left\{\varepsilon \in 2^{\omega} \mid \zeta(\varepsilon)\right.$ is odd $\leftrightarrow \eta$ is even $\}$ and $H_{1, \eta}=\check{G}_{1, \eta}$. Note that for $\eta=1$ and $\psi$ the identity: $\omega \rightarrow \omega$, the corresponding set is just $G_{1}$. 
Lemma 3. For each $\eta<\omega_{1}$, the set $G_{1, \eta}$ is a strategically complete set in $D_{\eta}\left(\Sigma_{1}^{0}\right)$.

Proof. For $\zeta<\eta$, let $G_{1, \eta}^{\zeta}=\left\{\varepsilon \in 2^{\omega} \mid \zeta(\varepsilon) \leqslant \zeta\right\}$. One easily checks that the $\left(G_{1, \eta}^{\zeta}\right)_{\zeta<\eta}$ form an increasing sequence of open sets, and $G_{1, \eta}=D_{\eta}\left(\left(G_{1, \eta}^{\zeta}\right)\right)$. So $G_{1, \eta} \in D_{\eta}\left(\Sigma_{1}^{0}\right)$. Suppose now $A \in D_{\eta}\left(\Sigma_{1}^{0}\right)$, say $A=D_{\eta}\left(\left(A_{\zeta}\right)\right)$, and pick trees $T_{\zeta}$ with $A_{\zeta}=\left\{\alpha \in 2^{\omega} \mid \exists n \alpha \uparrow_{n} \notin T_{\zeta}\right\}$. A winning strategy for II in $G_{w}\left(A, G_{1, \eta}\right)$ is given by: At stage $k$, II answers to $u$ played by $I$ by 0 iff $u \in T_{\psi_{0}(k)}$. One easily checks that it works.

We now define the games $J_{1, \eta}\left(A_{0}, A_{1}\right)$ corresponding to $D_{\eta}\left(\Sigma_{1}^{0}\right)$. The play is again the same, i.e. I produces $\varepsilon$ and II produces $(\alpha, \beta) \in 2^{\omega} \times \omega^{\omega}$. At stage $k$, say that position $u_{k}=\left(\varepsilon \uparrow_{k}, \alpha \uparrow_{k}, \beta \uparrow_{k}\right)$ is legal in $J_{1, \eta}\left(A_{0}, A_{1}\right)$ if setting $n_{k}=\inf \{n \leqslant k$ : $\left.\zeta\left(\varepsilon \uparrow_{n}\right)=\zeta\left(\varepsilon \uparrow_{k}\right)\right\}$ and $i_{k}=0$ if $\zeta\left(\varepsilon \uparrow_{k}\right)$ has the parity of $\eta$, and 1 if not, then $\left(\alpha \uparrow_{k-n_{k}},\left\langle\beta\left(n_{k}\right), \beta\left(n_{k}+1\right), \ldots, \beta(k-1)\right\rangle\right) \in T_{i_{k}}$. And player II wins $J_{1, \eta}\left(A_{0}, A_{1}\right)$ if all finite positions of the play are legal.

For $\zeta<\eta$ define open sets $C_{\zeta}^{\eta}$ in $2^{\omega} \times \Sigma$ by $(\alpha, \sigma) \in C_{\zeta}^{\eta} \leftrightarrow \exists u \in \omega^{<\omega} \quad(u$ is $(\sigma, \alpha)$-legal and the play $v$ of length $\mathrm{lh} u+1$ answered by I following $\sigma$ satisfies $\zeta(v) \leqslant \zeta)$, where of course $(\sigma, \alpha)$-legality refers to the game $J_{1, \eta}\left(A_{0}, A_{1}\right) \uparrow_{\alpha}$ (obtained as before by fixing $\alpha$ in the game $\left.J_{1, \eta}\left(A_{0}, A_{1}\right)\right)$.

Now let $C^{\eta}$ be $D_{\eta}\left(\left(C_{\zeta}^{\eta}\right)_{\zeta<\eta}\right)$.

Lemma 4. (i) Let $(\varepsilon, \alpha, \beta)$ be a win for II in $J_{1, \eta}\left(A_{0}, A_{1}\right)$. If $\varepsilon$ is in $H_{1, \eta}$, then $\alpha$ is in $A_{0}$. And if $\varepsilon$ is in $G_{1, \eta}$, then $\alpha$ is in $A_{1}$.

(ii) Suppose $\sigma$ is a winning strategy for player I in the game $J_{1, \eta}\left(A_{0}, A_{1}\right) \uparrow_{\alpha}$, for some given $\alpha \in 2^{\omega}$. Then if $\alpha \in A_{0}$, one has $(\alpha, \sigma) \in C^{\eta}$. And if $\alpha \in A_{1}$, one has $(\alpha, \sigma) \notin C^{\eta}$.

Proof. (i) Let $\zeta=\zeta(\varepsilon) \leqslant \eta$, and let $n_{\varepsilon}=$ least $n\left(\zeta\left(\varepsilon \uparrow_{n+1}\right)=\zeta\right)$ and $i_{\varepsilon}$ the corresponding $i_{n_{\varepsilon}}$. Let $\beta^{*}(i)=\beta\left(n_{\varepsilon}+i\right)$. From the definition of legal positions, one gets that $\left(\alpha, \beta^{*}\right) \in\left[T_{i_{\varepsilon}}\right]$. And clearly if $\varepsilon \in H_{1, \eta}, i_{\varepsilon}=0$ while if $\varepsilon \in G_{1, \eta}, i_{\varepsilon}=1$. This proves (i).

(ii) One easily checks that the sets $C_{\zeta}^{\eta}$ form an increasing sequence of open sets. We let $\alpha$ be fixed, and $\sigma$ be a winning strategy for I in $J_{1, \eta}\left(A_{0}, A_{1}\right) \uparrow_{\alpha}$. We argue by contradiction: Suppose first $\alpha \in A_{1}$ and $(\alpha, \sigma) \in C^{\eta}$. Pick $\beta$ with $(\alpha, \beta) \in\left[T_{1}\right]$, and let $\zeta=\min \left\{\zeta^{\prime}<\eta \mid(\alpha, \sigma) \in C_{\zeta^{\prime}}^{\eta}\right\}$. Pick $u$ of minimal length so that $u$ is $(\sigma, \alpha)$-legal and its answer $v$ by $\sigma$ satisfies $\zeta(v) \leqslant \zeta$. By minimality of $\zeta, \zeta(v)=\zeta$, and because $\zeta$ and $\eta$ have opposite parities, $v$ calls for starting a witness that $\alpha \in A_{1}$. Again by minimality any $(\sigma, \alpha)$-legal extension $u^{\prime}$ of $u$ will have as answer by $\sigma$ some $v^{\prime}$ with $\zeta\left(v^{\prime}\right)=\zeta$. Hence by induction one easily checks that $u^{\wedge} \beta$ is a play in $J_{1, \eta}\left(A_{0}, A_{1}\right) \uparrow_{\alpha}$ defeating $\sigma$, a contradiction which proves that $(\alpha, \sigma) \notin C^{\eta}$. The proof that $\alpha \in A_{0}$ implies $(\alpha, \sigma) \in C^{\eta}$ is similar, and we skip it.

Corollary 5. (i) If $\tau$ is a winning strategy for II in $J_{1, \eta}\left(A_{0}, A_{1}\right)$, the strategy $\tau^{*}$ for II in $G_{w}^{*}\left(H_{1, \eta} ; A_{0}, A_{1}\right)$ obtained by deleting the $\beta$ moves is also winning.

(ii) If $\sigma$ is a winning strategy for I in $J_{1, \eta}\left(A_{0}, A_{1}\right)$ and for $\alpha \in 2^{\omega}, \sigma_{\alpha}$ denotes the corresponding strategy in $J_{1, \eta} \uparrow_{\alpha}$, then $C_{\sigma}^{\eta}=\left\{\alpha \in 2^{\omega} \mid\left(\alpha, \sigma_{\alpha}\right) \in C^{\eta}\right\}$ is a $D_{\eta}\left(\Sigma_{1}^{0}\right)$ set which separates $A_{0}$ from $A_{1}$. 
3. Ramifications of closed games. We now present the kind of "unfolding" of games we need for inductively constructing the games $J_{\xi}$ and $J_{\xi, \eta}$. Let $\mathscr{J}$ be the set of all trees on $2 \times \omega$, i.e. subsets $J$ of $(2 \times \omega)^{<\omega}$ closed under restrictions, which we identify with closed (for II) games on $2 \times \omega$, as follows

$$
\text { I } \varepsilon(0) \quad \varepsilon(1)
$$

II

$\beta(0)$

I produces $\varepsilon \in 2^{\omega}$, II produces $\beta \in \omega^{\omega}$ and II wins if $(\varepsilon, \beta) \in[J]$, i.e. if $\forall n\left(\varepsilon \uparrow_{n}\right.$, $\left.\beta \uparrow_{n}\right) \in J$, so that $J$ is the set of legal positions in this game. The set $\mathscr{J}$ is compact, with the topology inherited from $2^{(2 \times \omega)^{<\omega}}$. We again denote by $\Sigma$ the set of functions $\sigma: \omega^{<\omega} \rightarrow 2$, which we view as strategies for I in games in $\mathscr{J}$. Again $\Sigma$ is compact for the usual product topology. For $\sigma$ in $\Sigma$ and $u \in \omega^{<\omega}$, we denote by $\bar{\sigma}(u)$ (of length $\operatorname{lh}(u)+1)$ the answer $\bar{\sigma}(u)=\sigma(\varnothing)^{\wedge} \sigma\left(u \uparrow_{1}\right)^{\wedge} \ldots{ }^{\wedge} \sigma(u)$ of player I following $\sigma$ against $u$. And if $J \in \mathscr{J}, \sigma \in \Sigma$, we say $u$ is $(J, \sigma)$-legal if $\left(\bar{\sigma}(u) \uparrow_{\mathrm{lh} u}, u\right)$ $\in J$. Similarly an infinite play $\beta \in \omega^{\omega}$ is $(J, \sigma)$-legal if for all $k \beta \uparrow_{k}$ is $(J, \sigma)$-legal, i.e. if the play $\beta$ defeats $\sigma$ in the game $J$. We then denote by $\bar{\sigma}(\beta)=\cup_{n} \bar{\sigma}\left(\beta \uparrow_{n}\right)$ the corresponding play of player I.

The notion of ramification is rather technical, so following the suggestions of the referee, we have added some comments to the formal definition, hoping that it will help the reader.

Definition 1. A ramification of games is a triple $R=\langle r, \rho, f\rangle$ consisting of three functions: the ramification function $r=\left(r_{0}, r_{1}\right): 2^{<\omega} \rightarrow 2^{<\omega} \times \omega^{<\omega}$, the projection function $\rho=\left(\rho_{0}, \rho_{1}\right): 2^{\omega} \rightarrow 2^{\omega} \times \omega^{\omega}$, and the filling-in function $f: J \times \Sigma \times \omega^{<\omega}$ $\rightarrow \omega^{<\omega}$, satisfying the following three properties (i), (ii), (iii):

(i) (a) For each $u \in 2^{<\omega} \operatorname{lh} r_{0}(u)=\operatorname{lh} r_{1}(u) \leqslant \operatorname{lh}(u)$,

(b) $r_{1}(u)$ is a strictly increasing function: $\operatorname{lh} r_{0}(u) \rightarrow \operatorname{lh} u$,

(c) For each $u \in 2^{<\omega},\left\{r\left(u \uparrow_{k}\right) \mid k \leqslant \operatorname{lh} u\right\}$ is a tree on $2 \times \omega$, i.e. $\forall k<\operatorname{lh}(r(u))$ $\exists j \leqslant \operatorname{lh} u \quad r\left(u \uparrow_{j}\right)=r(u) \uparrow_{k}$.

We can extend $r$ to a function (we still denote by $r)$ from $(2 \times \omega)^{<\omega}$ into $(2 \times \omega)^{<\omega}$ by

$$
r(u, v)=\left(r_{0}(u), v \circ r_{1}(u)\right)
$$

and by transposition, associate with $r$ a function $R: \mathscr{J} \rightarrow \mathscr{J}$, by $R(J)=\{(u, v) \in$ $\left.(2 \times \omega)^{<\omega}: \forall i \leqslant \operatorname{lh} u r\left(u \uparrow_{i}, v \uparrow_{i}\right) \in J\right\}$. The game $R(J)$ is called the ramification of $J$, or the ramified game associated with $J$ : in order to win $R(J)$ by playing $\beta$ against $\varepsilon$, I has to ensure that the tree $T_{\varepsilon, \beta}=\left\{r\left(\varepsilon \uparrow_{i}, \beta \uparrow_{i}\right): i \in \omega\right\}$ is a subtree of $J$.

Intuitively, at each position $(u, v)$ in the game $R(J)$, the two players are imagining that they are playing in $J$, at some fake position $\left(u^{*}, v^{*}\right)$ which is given by the function $r$, and $(u, v)$ is legal in $R(J)$ just in case $\left(u^{*}, v^{*}\right)$ is legal in $J$.

Note that the fake play $v^{*}$ of II is a subplay of $v$, which is determined by the sequence $\left\langle n_{0}, \ldots, n_{j-1}\right\rangle$ which depends only on I's play $u$ (via $r_{1}$ ). (See Figure 1.) This is an essential feature of a ramification: The idea is that I's play $u$ is just encoding (via $r_{1}$ ) some demands about II's beginning of a witness $v^{*}$, together with some $u^{*}$, which itself encodes some demands about II's beginning of a witness $v^{* *}$, together with some $u^{* *}$ and so on, in a (possibly transfinite) process to be described later. 
Note also that by extending $u$ in $R(J)$, player I does not automatically extend $u^{*}$ in $J$ : By following $r$, he may first erase some of the last moves of $u^{*}$ (and the corresponding values of the sequence $\left.\left\langle n_{0}, \ldots, n_{j-1}\right\rangle\right)$ and then extend the remainder of it. This is why we call $r$ a ramification.

(ii) For each $\varepsilon \in 2^{\omega}, \rho(\varepsilon)=\left(\rho_{0}(\varepsilon), \rho_{1}(\varepsilon)\right)$ is a branch through the tree $T_{\varepsilon}=$ $\left\{r\left(\varepsilon \uparrow_{i}\right): i \in \omega\right\}$.

Extend the function $\rho$ to a function (still denoted by $\rho$ ): $2^{\omega} \times \omega^{\omega} \rightarrow 2^{\omega} \times \omega^{\omega}$, defined by

$$
\rho(\varepsilon, \beta)=\left(\rho_{0}(\varepsilon), \beta \circ \rho_{1}(\varepsilon)\right) .
$$

Condition (ii) means that if players I and II have played $\varepsilon$ and $\beta$ in a run of some game $R(J)$, there corresponds at least one fake run $\rho(\varepsilon, \beta)$ in the game $J$. Hence in particular if II wins the run $(\varepsilon, \beta)$ in $R(J)$, then II also wins the run $\rho(\varepsilon, \beta)$ in $J$. But we do not impose a priori continuity (or convergence) conditions on how the fake run is obtained.

(iii) It follows from (ii) that it is harder for II to win $R(J)$ than to win $J$. We now introduce the last property of a ramification, to the effect of avoiding easy wins of player $\mathrm{I}$ in $R(J)$. This is done by using the filling-in function $f$.

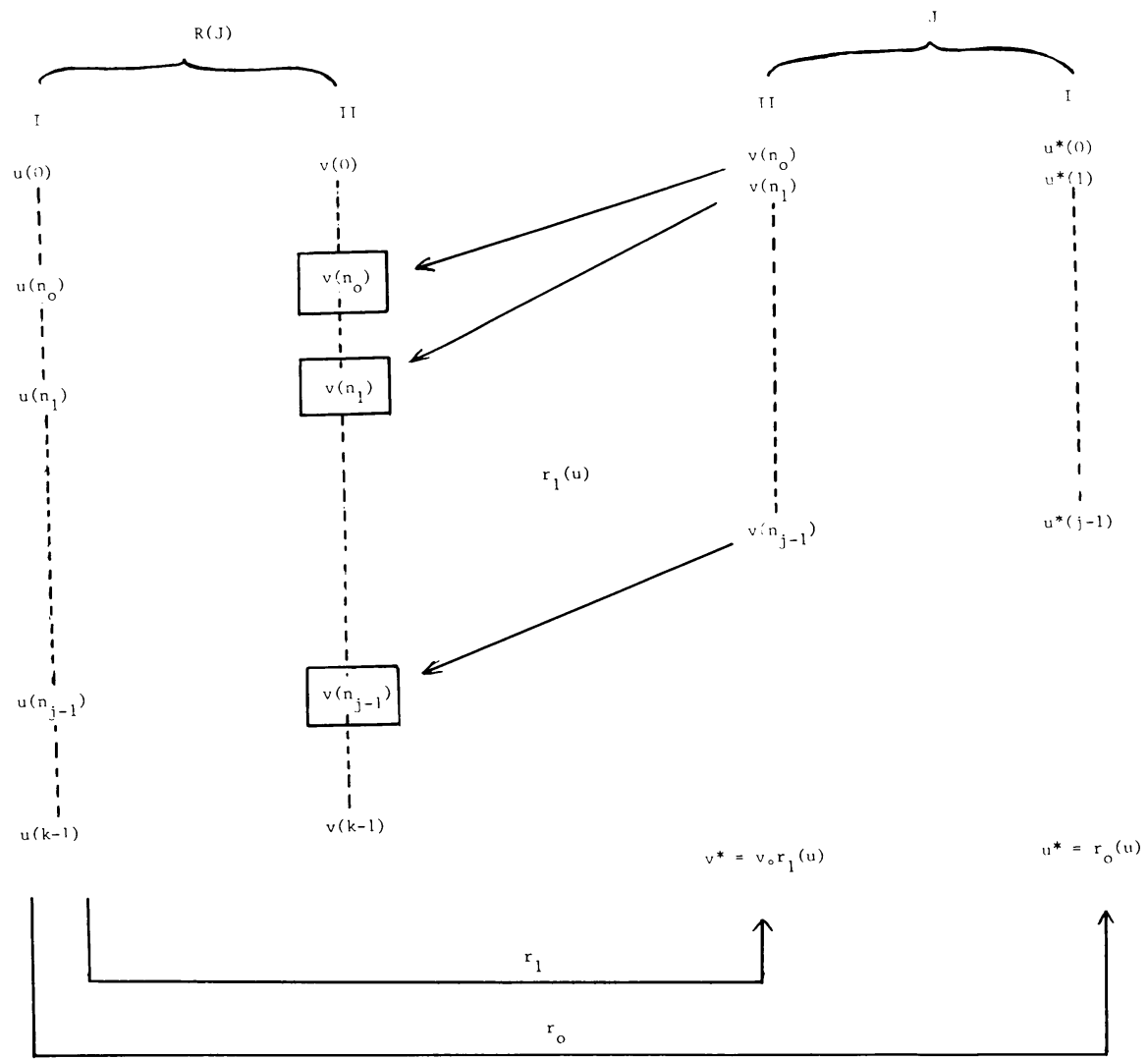

Figure 1 
Fix a game $J$, and a strategy $\sigma$ for player $\mathrm{I}$ in the game $R(J)$. The filling-in function $f$ allows to build a strategy $\sigma^{*}=F(J, \sigma)$ for I in $J$, as follows:

Step 1. (See Figure 2.) We first consider II in $R(J)$ beginning with $v_{0}=f(\varnothing)$ (of length $k$ ) and I answering via $\sigma$ some $u_{0}$ (of length $k+1$ ). It has to be that-(a) the positions up to $\left(u_{0}(0), \ldots, u_{0}(k-1)\right),\left(v_{0}(0), \ldots, v_{0}(k-1)\right)$ are legal in $R(J)$ -(b) The fake position $r_{0}\left(u_{0}\right)$ is of length 1 -and this is by definition the first move of I in $J$ following $\sigma^{*}=F(J, \sigma)$-and (c) the function $r_{1}\left(u_{0}\right)$ sends 0 to $k$, so that for any further play $n$ of II in $R(J)$, the corresponding fake play in $J$ is just $(\varepsilon(0), n)$.

Step 2. (See Figure 3.) Let II then play some integer $\beta(0)=n$ in $J$. There are two cases. If $\langle(\varepsilon(0), \beta(0))\rangle \notin J$, II has already lost in $J$ and we may ask I to play 0 from now on.

If now $\langle(\varepsilon(0), \beta(0))\rangle \in J$, we do the following: In $R(J)$ we let II play $\beta(0)$, and then $v_{1}=f(\langle\beta(0)\rangle)$, and let I answer some $u_{1}$ using $\sigma$ (with $\operatorname{lh} u_{1}=l+1=\operatorname{lh} v_{1}+$ $1)$. Notice that the position $\left(u_{0}, v_{0}^{\wedge} \beta(0)\right)$ is by the hypothesis legal in $R(J)$, as its fake position is $(\langle\varepsilon(0)\rangle,\langle\beta(0)\rangle) \in J$. It now has to be that-(a) the position $\left(u_{0}^{\wedge}\left\langle u_{1}(0), \ldots, u_{1}(l-1)\right\rangle, v_{0}^{\wedge} \beta(0)^{\wedge} v_{1}\right)$ is $R(J)$-legal - (b) the $r_{0}$-transform of $u_{0}^{\wedge} u_{1}$ is a sequence $\langle\varepsilon(0), \varepsilon(1)\rangle$ of length 2 which extends $\langle\varepsilon(0)\rangle$-hence allows us to define in $J$ the answer to II's play $\beta(0)$-and (c) the function $r_{1}\left(u_{0}^{\wedge} u_{1}\right)$ sends 0 to $k$ (thus extends $r_{1}\left(u_{0}\right)$ ) and 1 to $k+l+1$, so that for any further play $p$ of II in $R(J)$, the corresponding fake play in $J$ is $(\langle\varepsilon(0), \varepsilon(1)\rangle,\langle\beta(0), p\rangle)$.

And so on: Suppose that we have defined the strategy $\sigma^{*}$ for all plays $\langle\beta(0), \ldots, \beta(k-1)\rangle$ of length $k$, of II in $J$ so that we know the answer $\langle\varepsilon(0), \ldots, \varepsilon(k)\rangle$ of player I. And let $\beta(k)$ be some further play of II in $J$. If the new position is not $J$-legal, $\sigma^{*}$ calls for 0 . If it is legal, we imagine the following plays in $R(J)$ :

Using the filling-in function, II plays $f(\varnothing)$, then $\beta(0)$, then $f(\langle\beta(0)\rangle)$, then $\beta(1)$, then $f(\langle\beta(0), \beta(1)\rangle), \ldots$, then $\beta(k-1)$, then $f(\langle\beta(0), \ldots, \beta(k-1)\rangle)$, then $\beta(k)$, then $f(\langle\beta(0), \ldots, \beta(k)\rangle)$, constructing some sequence $v$; I answers by $\sigma$ some sequence $u$ one step longer than $v$, say $u=u^{\prime \wedge}\langle q\rangle$. We first impose that $\left(u^{\prime}, v\right)$ is $R(J)$-legal. Next, consider the corresponding plays in $J$, obtained using $r$. We impose the condition that I's play $r_{0}(u)$ is a one-step extension of $\langle\varepsilon(0), \ldots, \varepsilon(k)\rangle$ by some $\varepsilon(k+1)$, which by definition is $\sigma^{*}(\langle\beta(0), \ldots, \beta(k)\rangle)$. Moreover the function $r_{1}$ sends $i<k$ to the position of $\beta(i)$ in $v$, and sends $k$ to lh $v$, so that for any further play $m$ of II in $R(J)$, the fake play in $J$ corresponding to $\left(u, v^{\wedge} m\right)$ is $(\langle\varepsilon(0), \ldots, \varepsilon(k+1)\rangle,\langle\beta(0), \ldots, \beta(k), m\rangle)$.

Formally, if $J \in \mathscr{J}$ and $\sigma \in \Sigma$ the filling-in function $f=f(J, \sigma)$ associates with each sequence $v$ (viewed as played by II in $J$ ) a sequence $f(v) \in \omega^{<\omega}$ such that if we set

$$
\bar{f}(v)=f(\varnothing)^{\wedge} v(0)^{\wedge} f\left(v \uparrow_{1}\right)^{\wedge} v(1)^{\wedge} \cdots{ }^{\wedge} v(\operatorname{lh} v-1)^{\wedge} f(v)
$$

and

$$
\bar{f}^{*}(v)=f(\varnothing)^{\wedge} v(0)^{\wedge} f\left(v \uparrow_{1}\right)^{\wedge} v(1)^{\wedge} \cdots \wedge v(\operatorname{lh} v-1)
$$




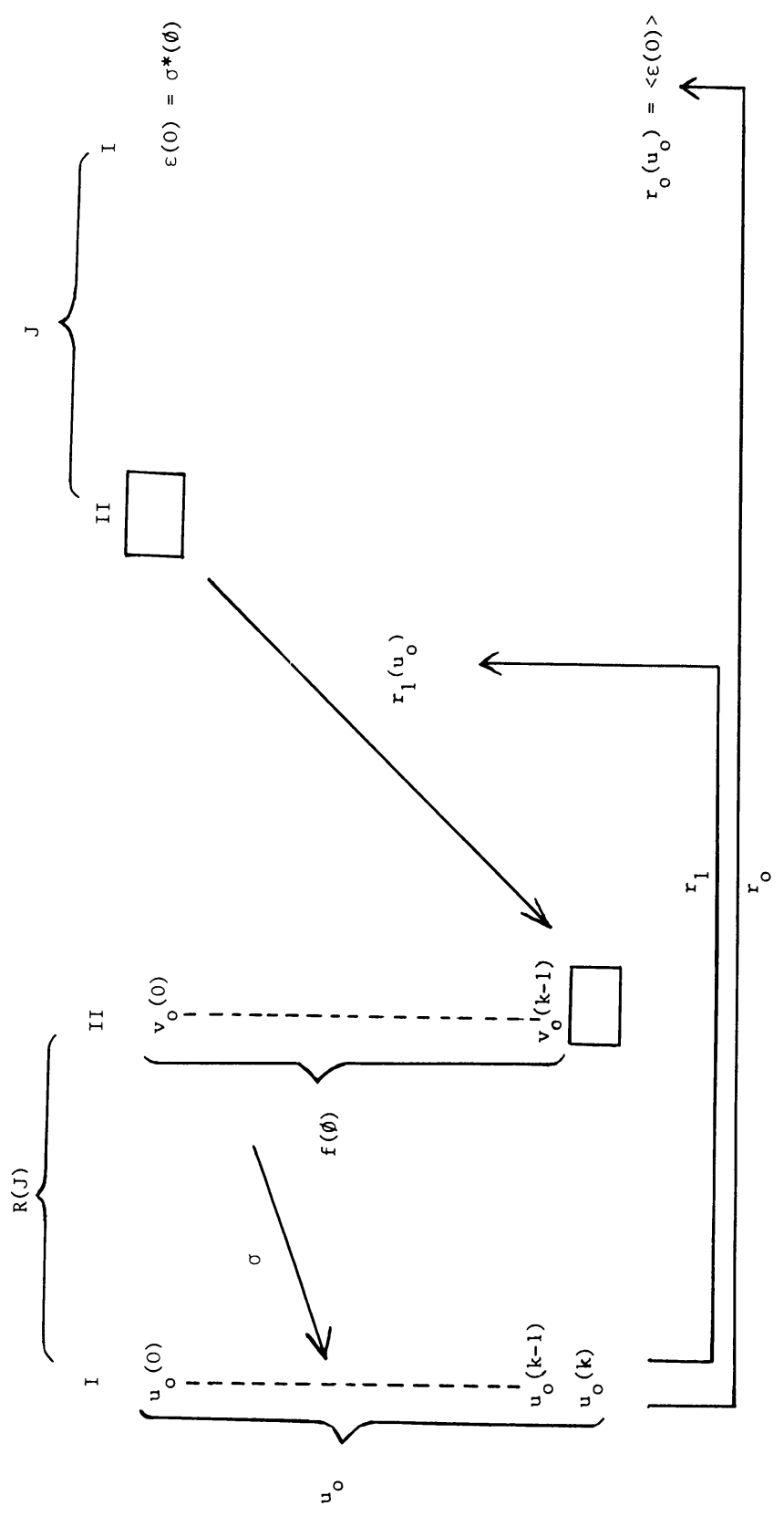

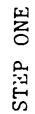

盝 


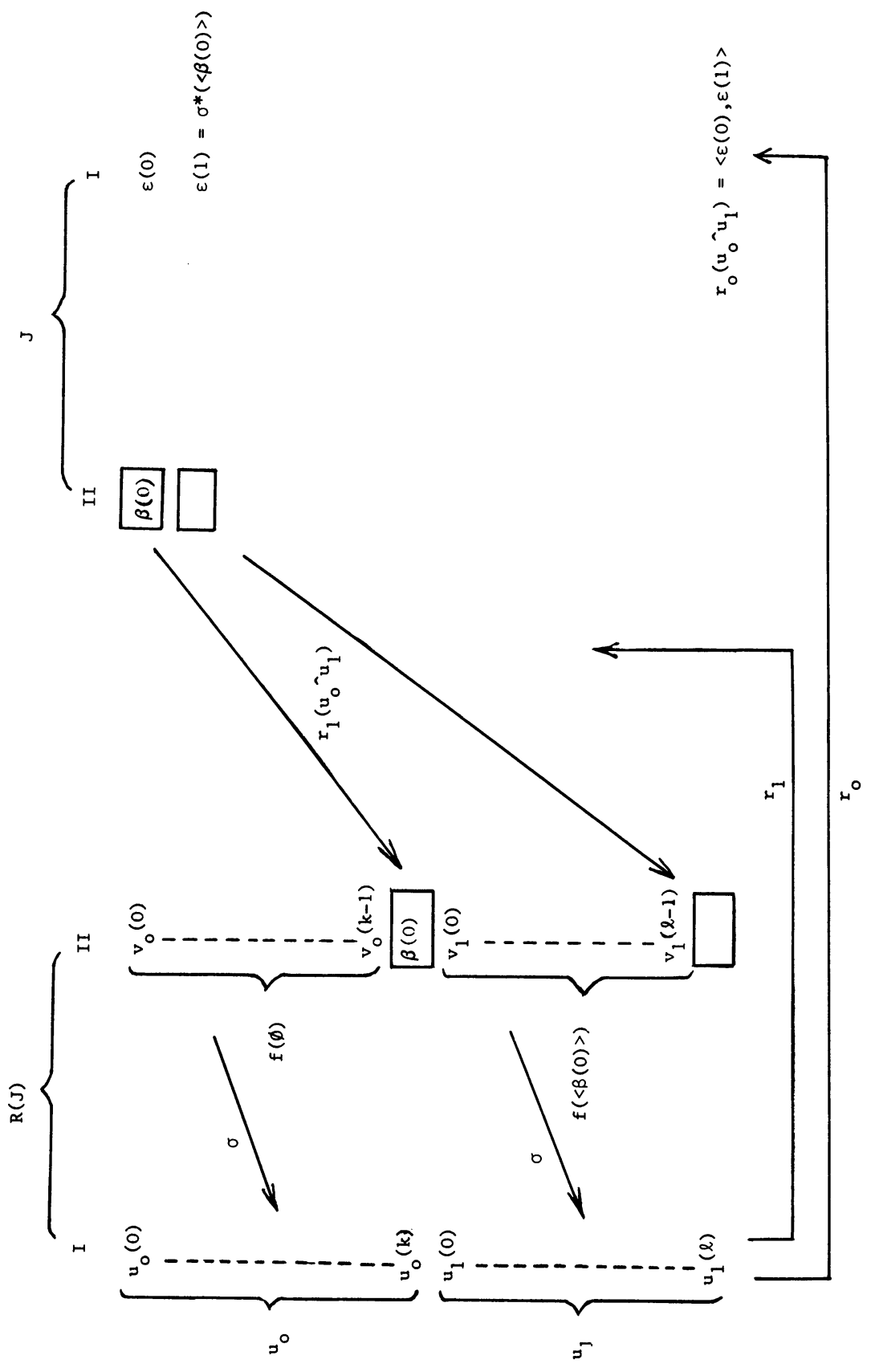

m 
we have

(a) If $\bar{f}^{*}(v)$ is $(R(J), \sigma)$-legal, so is $\bar{f}(v)$.

(b) If $\bar{f}(v)$ is $(R(J), \sigma)$-legal, and $u=\bar{\sigma}(\bar{f}(v))$ is the corresponding play (of length $\operatorname{lh} \bar{f}(v)+1$ ) of I following $\sigma$ in $R(J)$, then $\operatorname{lh} r(u)=\operatorname{lh} v+1$; the function $r_{1}(u): \operatorname{lh} r(u) \rightarrow \operatorname{lh} u$ is given by

(so that for any $m \in \omega r_{1}\left(u, \bar{f}(u)^{\wedge} m\right)=v^{\wedge} m$ ).

$$
r_{1}(u)(i)=\ln \left(\bar{f}\left(v \uparrow_{i}\right)\right) \text { for } i \leqslant \operatorname{lh} v
$$

(c) If $v^{*}=v \uparrow_{\mathrm{lh} v^{-1}}$ and $u^{*}=\bar{\sigma}\left(\bar{f}\left(v^{*}\right)\right)$, then $r_{0}(u)$ is an extension of $r_{0}\left(u^{*}\right)$.

By the properties of the filling-in function, one can associate with $f$ a function $F$ : $\mathscr{J} \times \Sigma \rightarrow \sum$ by

$$
F(J, \sigma)(v)=\left\{\begin{array}{l}
r_{0}(\bar{\sigma}(\bar{f}(v)))(\operatorname{lh}(v)) \text { if } \bar{f}(v) \text { is }(R(J), \sigma) \text {-legal, } \\
0 \text { otherwise, }
\end{array}\right.
$$

which associates with each strategy $\sigma$ in $R(J)$ a strategy $\sigma^{*}=F(J, \sigma)$ in $J$, with the property that for each $\left(J, \sigma^{*}\right)$-legal play $v$ the corresponding play $\bar{f}(v)$ is $(R(J), \sigma)$ legal, and satisfies for each $m \in \omega$

$$
r\left(\overline{\boldsymbol{\sigma}}(\bar{f}(v)), \bar{f}(v)^{\wedge} m\right)=\left(\overline{\boldsymbol{\sigma}}^{*}(v), v^{\wedge} m\right),
$$

so that if $\beta$ defeats $\sigma^{*}$ in $J, \bar{f}(\beta)=\bigcup\left\{\bar{f}\left(\beta \uparrow_{k}\right): k \in \omega\right\}$ defeats $\sigma$ in $R(J)$; hence if $\sigma$ is winning for I in $R(J)$ the strategy $\sigma^{*}=F(J, \sigma)$ is winning for I in $J$.

Let $\eta$ be a given countable ordinal. Recall that a function $f: E \rightarrow F$ (where $E, F$ are Polish spaces) is of class $\eta$ if for all open sets $U \subset F$ the set $f^{-1}(U)$ is $\Sigma_{1+\eta}^{0}$ in $E$ (so that continuous functions are given class 0 ). In this section we shall prove the following theorem on the existence of ramifications.

THEOREM 2. For each countable ordinal $\eta$, there exists a ramification $\underline{R}_{\eta}=(r, \rho, f)$ such that

(i) $\rho: 2^{\omega} \rightarrow 2^{\omega} \times \omega^{\omega}$ and $f: \mathscr{J} \times \Sigma \times \omega^{<\omega} \rightarrow \omega^{<\omega}$ are of class $\eta$.

(ii) The set $H_{1+\eta}=\rho_{0}^{-1}\left(H_{1}\right)=\left\{\varepsilon \in 2^{\omega}: \rho_{0}(\varepsilon)=\underline{0}\right\}$ is a strategically complete $\Pi_{1+\eta}^{0}$ set .

We shall prove Theorem 2 by iterating a basic one-step ramification, using composition at successor stages, and a direct limit process at limit stages.

LEMMA 3. There exists a ramification $\underline{R}=(r, \rho, f)$, we call the basic ramification, which satisfies

(i) $\rho$ and $f$ are first class functions.

(ii) If we define $\langle n, i\rangle=\frac{1}{2}(i+n)(i+n+1)+i$ (with inverse maps: $\omega \rightarrow \omega$, $(\cdot)_{0}$ and $\left.(\cdot)_{1}\right)$, then the function $\rho_{0}: 2^{\omega} \rightarrow 2^{\omega}$ is given by

$$
\rho_{0}(\varepsilon)(n)=1 \leftrightarrow \forall i \in \omega \varepsilon(\langle n, i\rangle)=0 .
$$

Proof. For commodity, extend the function $\rho_{0}: 2^{\omega} \rightarrow 2^{\omega}$ to $2^{<\omega}$ by setting $\rho_{0}(u)=\rho_{0}\left(u^{\wedge} \underline{0}\right)$. We now define by induction the ramification function $r$. The sequence $r_{0}(u)$ will be some initial segment of $\rho_{0}(u)$, so it is enough to inductively define $\operatorname{lh}(r(u))$ and $r_{1}(u): \operatorname{lh}(r(u)) \rightarrow \operatorname{lh} u$. Start with $\operatorname{lh} r(\varnothing)=0$ and $r_{1}(\varnothing)=\varnothing$. Suppose now $\operatorname{lh} r(u), r_{0}(u)=\rho_{0}(u) \uparrow_{\operatorname{lh} r(u)}$ and $r_{1}(u)$ have been defined, and let $i=0$ or 1 . We want to define $r\left(u^{\wedge} i\right)$. 
Case 1. The sequence $r_{0}(u)$ is an initial segment of $\rho_{0}\left(u^{\wedge} i\right)$. In this case, let $\operatorname{lh} r\left(u^{\wedge} i\right)=\operatorname{lh} r(u)+1$, and $r_{1}\left(u^{\wedge} i\right)=r_{1}(u)^{\wedge} \operatorname{lh} u$.

Case 2. Otherwise. By the definition of $\rho_{0}$, this means that one has $i=1$, and setting $k_{0}=(\operatorname{lh} u)_{0}$, that $r_{0}(u)\left(k_{0}\right)=1$ but $\rho_{0}\left(u^{\wedge} 1\right)\left(k_{0}\right)=0$. In this case, let $\operatorname{lh}\left(r\left(u^{\wedge} 1\right)\right)=k_{0}+1$, and $r_{1}\left(u^{\wedge} 1\right)=r_{1}(u) \uparrow_{k_{0}}{ }^{\wedge} \operatorname{lh} u$.

One easily checks that $r_{1}(u)$ is strictly increasing, and that $\left\{r\left(u \uparrow_{i}\right): i \leqslant \operatorname{lh} u\right\}$ forms a tree in $(2 \times \omega)^{<\omega}$.

We now claim that for each $\varepsilon \in 2^{\omega}$ the sequence $\left(r\left(\varepsilon \uparrow_{k}\right)\right)_{k \in \omega}$ converges, and moreover $\lim _{k \rightarrow \infty} r_{0}\left(\varepsilon \uparrow_{k}\right)=\rho_{0}(\varepsilon)$. The reason is that there is at most one change (from 1 to 0 ) for $\rho_{0}\left(\varepsilon \uparrow_{k}\right)(0)$, and after it the $r\left(\varepsilon \uparrow_{k}\right)$ will have constant value at 0 , the value of $r_{0}\left(\varepsilon \uparrow_{k}\right)(0)$ being $\rho_{0}(\varepsilon)(0)$, and for bigger $k$ there is at most one change (from 1 to 0 ) for $\rho_{0}\left(\varepsilon \uparrow_{k}\right)(1)$, and after it the $r\left(\varepsilon \uparrow_{k}\right)$ will have the same value at 1 , the value of $r_{0}\left(\varepsilon \uparrow_{k}\right)(1)$ being $\rho_{0}(\varepsilon)(1)$, etc... So we define $\rho(\varepsilon)=\lim _{k \rightarrow \infty} r\left(\varepsilon \uparrow_{k}\right)$, and $\rho(\varepsilon)$ is a (in fact the unique) branch through the tree $T_{\varepsilon}$. And clearly $\rho$ is a first class function, as the pointwise limit of the continuous functions $\varepsilon \mapsto r\left(\varepsilon \uparrow_{k}\right)$.

It remains to define the filling-in function $f(J, \sigma, u)$. Fix $J \in \mathscr{J}$ and $\sigma \in \Sigma$. The function $f(J, \sigma, u)=f(u)$ is defined by induction on lh $u$. First let $u=\varnothing$. Consider the set

$$
L_{\varnothing}=\left\{w \in \omega^{<\omega}: w \text { is }(R(J), \sigma) \text {-legal and }(\operatorname{lh} w)_{0}=0\right\} \text {. }
$$

As $\varnothing \in L_{\varnothing}, L_{\varnothing}$ is nonempty. There are two cases.

Case 1. For all $w \in L_{\varnothing}, \sigma(w)=0$. Let then $f(\varnothing)=\varnothing$.

Case 2. Otherwise. Pick (in some canonical way) some $u_{\varnothing}$ in $L_{\varnothing}$ among the $w$ 's of minimal length such that $\sigma(w)=1$, and let $f(\varnothing)=u_{\varnothing}$. One easily checks that in both cases $f(\varnothing)$ is $(R(J), \sigma)$-legal, and for any $m \in \omega r_{1}\left(\bar{\sigma}(f(\varnothing)), f(\varnothing)^{\wedge} m\right)=$ $\langle m\rangle$.

Moreover for any $(R(J), \sigma)$-legal extension $v$ of $f(\varnothing)^{\wedge} m$ (if any), the sequence $r\left(\bar{\sigma}(v) \uparrow_{\mathrm{lh} v}, v\right)$ will extend $r\left(\bar{\sigma}(f(\varnothing)), f(\varnothing)^{\wedge} m\right)$. Suppose now $f(u)$ has been defined so that $\bar{f}(u)$ is $(R(J), \sigma)$-legal, for any $m \in \omega r_{1}\left(\bar{\sigma}(\bar{f}(u)), \bar{f}(u)^{\wedge} m\right)=u^{\wedge} m$, and that for any $(R(J), \sigma)$-legal extension $v$ of $\bar{f}(u)^{\wedge} m$ (if any) the sequence $r\left(\bar{\sigma}(v) \uparrow_{\mathrm{lh} v}, v\right)$ extends $r\left(\overline{\boldsymbol{\sigma}}(\bar{f}(u)), \bar{f}(u)^{\wedge} m\right)$. Let $v=u^{\wedge} m$. If $\bar{f}(u)^{\wedge} m$ is not $(R(J), \sigma)$-legal, i.e. $r\left(\bar{\sigma}(\bar{f}(u)), \bar{f}(u)^{\wedge} m\right)=(\overline{F(J, \sigma)}(u), v)$ is not in $J$, i.e. $v$ is not $\left(J, F(J, \sigma)\right.$ )-legal, we can define $f(v)$ (and $f\left(v^{\prime}\right)$ for any extension $v^{\prime}$ of $v$ ) to be anything, say $\varnothing$. So assume $v$ is $(J, F(J, \sigma))$-legal, i.e. $\bar{f}(u)^{\wedge} m$ is $(R(J), \sigma)$-legal. Let $L_{v}=\left\{w \mid w\right.$ extends $\bar{f}(u)^{\wedge} m$ and $w$ is $(R(J), \sigma)$-legal and $\operatorname{lh} r(\bar{\sigma}(w))=\operatorname{lh} v+$ $1\}$. By the induction hypothesis, $\bar{f}(u)^{\wedge} m \in L_{v}$. Again there are two cases.

Case 1. If for all $w \in L_{v} r_{0}(\bar{\sigma}(w))(\operatorname{lh} v)=r_{0}\left(\bar{\sigma}\left(\bar{f}(u)^{\wedge} m\right)\right)(\operatorname{lh} v)$, we set $f(v)=\varnothing$.

Case 2. Otherwise. Pick $w \in L_{v}$ of minimal length so that $r_{0}(\bar{\sigma}(w))(\operatorname{lh} v)$ is different from $r_{0}\left(\bar{\sigma}\left(\bar{f}(u)^{\wedge} m\right) \text { ) (lh } v \text { ) (this happens only if (lhw }\right)_{0}=\operatorname{lh} v$, and $\sigma(w)$ $=1$ ), and let $f(v)$ be the unique sequence $t$ such that $\bar{f}(u)^{\wedge} m^{\wedge} t$ is just $w$. Then $\bar{f}(v)=w$. In both cases, one easily checks that the inductive properties are satisfied, and we are done. An easy computation shows that $f$ is a class 1 function. 
LEMMA 4. Let $\underline{R}^{0}=\left(r^{0}, \rho^{0}, f^{0}\right)$ and $\underline{R}^{1}=\left(r^{1}, \rho^{1}, f^{1}\right)$ be two ramifications. One can define a ramification $\underline{R}=\underline{R}^{1} \circ \underline{R}^{0}=(r, \rho, f)$, the composition of $\underline{R}^{0}$ and $\underline{R}^{1}$, by the equations

(i) $r_{0}(u)=r_{0}^{1}\left(r_{0}^{0}(u)\right)$ and $r_{1}(u)=r_{1}^{0}(u) \circ r_{1}^{1}\left(r_{0}^{0}(u)\right)$ (so that for any $J \in \mathscr{J}, R(J)$ $\left.=R^{0}\left(R^{1}(J)\right)\right)$.

(ii) $\rho_{0}(\varepsilon)=\rho_{0}^{1}\left(\rho_{0}^{0}(\varepsilon)\right)$ and $\rho_{1}(\varepsilon)=\rho_{1}^{0}(\varepsilon) \circ \rho_{1}^{1}\left(\rho_{0}^{0}(\varepsilon)\right)$ (so that for any play $(\varepsilon, \beta)$, $\left.\rho(\varepsilon, \beta)=\rho^{1}\left(\rho^{0}(\varepsilon, \beta)\right)\right)$.

(iii) for all $(J, \sigma)$ in $\mathscr{J} \times \Sigma, f^{0}=f^{0}\left(R^{1}(J), \sigma\right), f^{1}=f^{1}\left(J, F^{0}\left(R_{1}(J), \sigma\right)\right), f=$ $f(J, \sigma)$ is implicitly defined, for $u \in \omega^{<\omega}$ as the unique sequence such that

$$
\overline{f^{0}}\left(\overline{f^{1}}(u)\right)=\overline{f^{0}}\left(\overline{f^{1}} *(u)\right)^{\wedge} f(u)
$$

( so that on legal plays, the strategy $F(J, \sigma)$ satisfies

$$
F(J, \sigma)=F^{1}\left(J, F^{0}\left(R^{1}(J), \sigma\right)\right) .
$$

SKETCH OF PROOF. For each position $(u, v)$ in $R(J)=R^{0}\left(R^{1}(J)\right)$, the players first imagine a fake position $\left(u^{*}, v^{*}\right)$ in $R^{1}(J)$ using $r^{0}$, and then a fake position $\left(u^{* *}, v^{* *}\right)$ in $J$ using $r^{1}$, and $(u, v)$ is legal in $R(J)$ just in case $\left(u^{* *}, v^{* *}\right)$ is legal in $J$. It is clear that $r$ as defined above is a ramification function, and that for each $\varepsilon \in 2^{\omega}$, the tree $T_{\varepsilon}$ corresponding to $r$ is obtained by first ramifying $\left\{\varepsilon \uparrow_{k}: k \in \omega\right\}$ using $r^{0}$ to get $T_{\varepsilon}^{0}$, and then ramifying $T_{\varepsilon}^{0}$ using $r^{1}$ to get $T_{\varepsilon}$. But as $\rho^{0}(\varepsilon)$ is a branch through $T_{\rho_{0}(\varepsilon)}^{1}, \rho(\varepsilon)=\rho^{1}\left(\rho^{0}(\varepsilon)\right)$ is a branch through $T_{\varepsilon}$.

It remains to check that the function $f$ defined in the statement above satisfies the conditions for being a filling-in function for $r$. We leave the reader at this point with the contemplation of the diagram on Figure 4.

The next lemma is easy to check and left to the reader.

Lemma 5. Let $\underline{R}=(r, \rho, f)$ be a ramification, and $k$ be some integer. One can define a ramification $T_{k} \underline{R}=\left(r^{\prime}, \rho^{\prime}, f^{\prime}\right)$, the translated ramification by $k$, by the equations

(i) for $u \in \omega^{i}, i \leqslant k r_{0}^{\prime}(u)=u$ and $r_{1}^{\prime}(u)$ is the identity (lh $\left.u \rightarrow \operatorname{lh} u\right)$; for $u=$ $u \uparrow_{k}^{\wedge} v, v \in \omega^{<\omega}, r_{0}^{\prime}(u)=u \uparrow_{k}^{\wedge} r_{0}(v)$ and $r_{1}^{\prime}(u)=\langle 0,1, \ldots, k-1\rangle^{\wedge} r_{1}(v)$,

(ii) for $\varepsilon=\varepsilon \uparrow_{k} \wedge \varepsilon^{\prime} \in 2^{\omega}, \rho^{\prime}(\varepsilon)=\left(\varepsilon \uparrow_{k},\langle 0, \ldots, k-1\rangle\right)^{\wedge} \rho\left(\varepsilon^{\prime}\right)$,

(iii) for $u \in \omega^{<k}, f^{\prime}(J, \sigma, u)=\varnothing$; and for $u=u \uparrow_{k}^{\wedge} v, f^{\prime}(J, \sigma, u)=f(J, \sigma, v)$ (so that for each $J \in \mathscr{J}$, and $\sigma \in \Sigma$, the strategies $\sigma$ and $F^{\prime}(J, \sigma)$ agree on legal moves in $J\left(=\right.$ in $\left.R^{\prime} J\right)$ of length $\left.\leqslant k\right)$.

Lemma 6. Let $\left(\underline{R}_{i}\right)_{i \in \omega}$ be a sequence of ramifications, and associate with this sequence an inductive system, by setting $\underline{R}_{i, i+1}=T_{i} \underline{R}_{i}$, and by induction, for $k>i$, $\underline{R}_{i, k+1}=\underline{R}_{k, k+1} \circ \underline{R}_{i, k}$. Then one can define a sequence $\left(\underline{R}_{i, \omega}\right)_{i \in \omega}$ of ramifications such that for $i<j<\omega$

$$
\underline{R}_{i, \omega}=\underline{R}_{j, \omega} \circ \underline{R}_{i, j}
$$


which is given by the equations

(i) $r^{(i, \omega)}(u)=r^{(i, i+\operatorname{lh} u)}(u)$,

(ii) $\rho^{(i, \omega)}(\varepsilon)=\lim _{n \rightarrow \infty} \rho^{(i, n)}(\varepsilon)$,

(iii) $f^{(i, \omega)}(J, \sigma, u)=f^{(i, i+\operatorname{lh} u)}(J, \sigma, u)$.

SKETCH OF PROOF. Notice first that as $r_{k, k+1}$ is the identity on sequences of length $\leqslant k$, we have $r^{(i, \omega)}(u)=r^{(i, j)}(u)$ for all big enough $j$, hence easily $r^{(i, \omega)}$ satisfies the properties for being a ramification function. Similarly $\rho^{(i, n)}(\varepsilon) \uparrow_{k}$ is constant, for $n$ bigger than $i$ and $k$, and is a finite branch through $\left\{r^{(i, n)}\left(\varepsilon \uparrow_{j}\right) \mid j \in \omega\right\} \cap \omega^{\leqslant k}=$ $\left\{r^{(i, \omega)}\left(\varepsilon \uparrow_{j}\right) \mid j \in \omega\right\} \cap \omega^{\leqslant k}$, so that $\rho^{(i, \omega)}(\varepsilon)=\lim _{n \rightarrow \infty} \rho^{(i, n)}(\varepsilon)$ exists and is a branch through $\left\{r^{(i, \omega)}\left(\varepsilon \uparrow_{j}\right) \mid j \in \omega\right\}$. Finally notice that for $n<n^{\prime}$, and $J \in \mathscr{J}$, the games $R^{i, n}(J)$ and $R^{i, n^{\prime}}(J)$ have the same legal positions of length $\leqslant n$, and for any

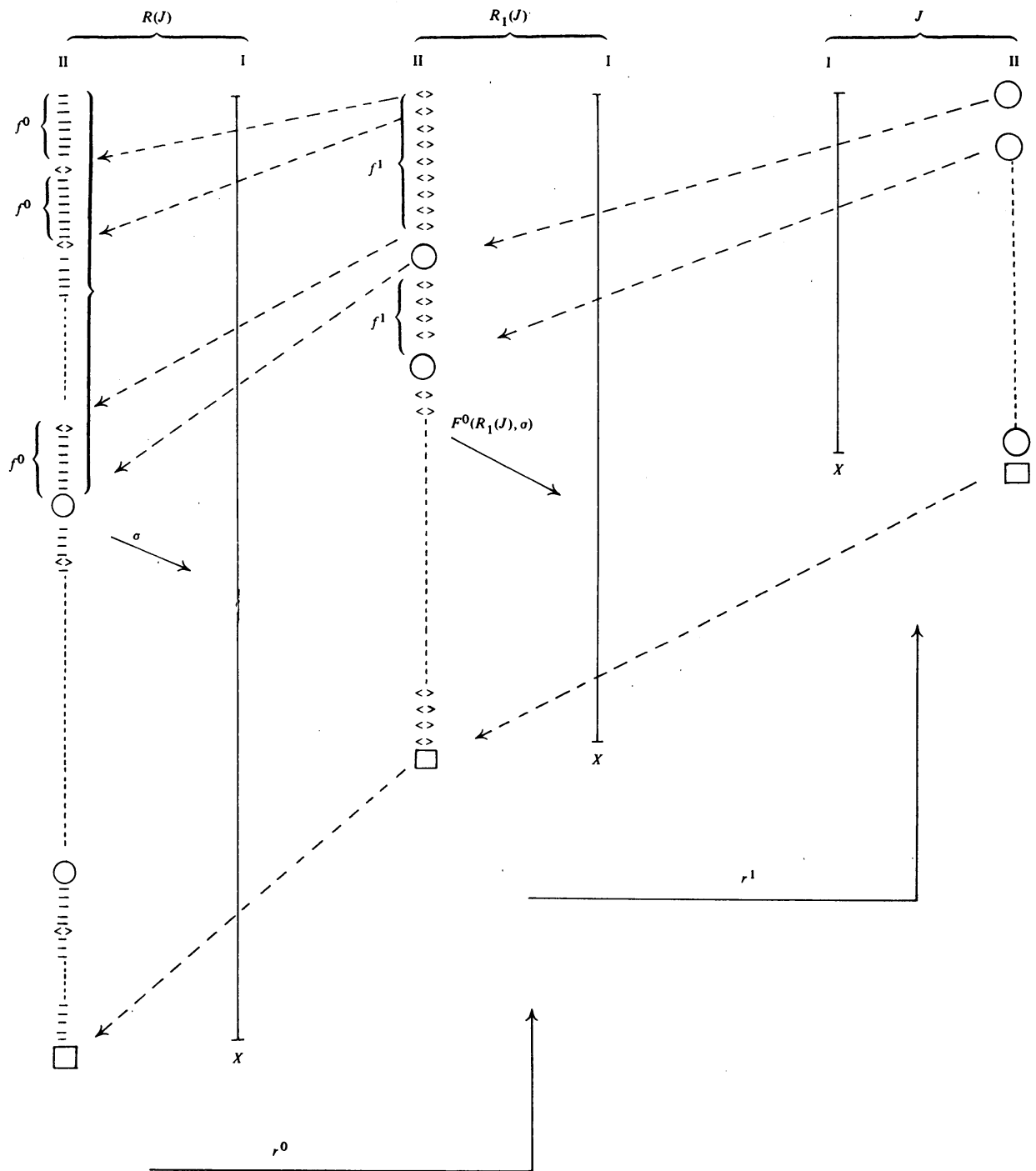

Figure 4 
$\sigma \in \Sigma$, if we consider $\sigma$ as a strategy in $R^{i, n}(J)$ and infer the corresponding $F^{i, n}(J, \sigma)$, or if we consider $\sigma$ as a strategy in $R^{i, n^{\prime}}(J)$ and infer $F^{i, n^{\prime}}(J, \sigma)$, we get two strategies which coincide on legal moves in $J$ of length $\leqslant n$. One easily checks using these facts that $f^{(i, \omega)}(J, \sigma, u)$ satisfies the properties of filling-in functions, and that moreover the corresponding strategy $F^{i, \omega}(J, \sigma)$ is (on legal moves) the limit of the strategies $F^{i, n}(J, \sigma)$. The commutativity of the diagram is left to the reader.

We are now in a position to prove Theorem 2. Of course, the main difficulty is to ensure property (ii) of $\underline{R}^{\eta}=\left(r^{\eta}, \rho^{\eta}, f^{\eta}\right)$ that $H_{1+\eta}=\left(\rho_{0}^{\eta}\right)^{-1}(\underline{0})$ be strategically complete in $\Pi_{1+\eta}^{0}$.

To do this, we will inductively prove a bit more about the functions $\rho_{0}^{\eta}$. Say that a function $\varphi: 2^{\omega} \rightarrow 2^{\omega}$ is an independent $\eta$-function if

(i) For some function $\pi: \omega \rightarrow \omega$, the value $\varphi(\varepsilon)(k)$, for each $k \in \omega$, depends only on the values of $\varepsilon$ on $\pi^{-1}(k)$.

(ii) If $\eta$ is successor, say $\eta=\xi+1$, then for each $k \in \omega\left\{\varepsilon \in 2^{\omega} \mid \varphi(\varepsilon)(k)=1\right\}$ is strategically complete $\Pi_{1+\xi}^{0}$

(iii) If $\eta$ is limit, then for some sequence $\left(\xi_{n}\right)$ with $\xi_{n}<\eta$ and $\sup _{n} \xi_{n}=\eta$. $\left\{\varepsilon \in 2^{\omega} \mid \varphi(\varepsilon)(k)=1\right\}$ is for any $k \in \omega$ a strategically complete $\Pi_{1+\xi_{h}}^{0}$ set.

We will prove that we can choose the $\underline{R}^{\eta}$ 's so that $\rho_{0}^{\eta}$ is an independent $\eta$-function.

We first need a lemma.

Lemma 7. Let $\pi$ be some function: $\omega \rightarrow \omega,\left(A_{n}, \xi_{n}\right)_{n \in \omega}$ a sequence such that

(i) $A_{n} \subset 2^{\omega}$, and the fact that $\varepsilon \in A_{n}$ depends only on $\varepsilon \uparrow \pi^{-1}(n)$.

(ii) $A_{n}$ is strategically complete $\Pi_{1+\xi_{n}}^{0}$.

Let $A=\bigcap_{n}\left(A_{n}^{\vee}\right)$, and $\xi=\sup _{n}\left(\xi_{n}+1\right)$. Then $A$ is a strategically complete $\Pi_{1+\xi}^{0}$ set.

Proof. The set $A$ is clearly $\Pi_{1+\xi}^{0}$. Suppose $B$ is some other $\Pi_{1+\xi}^{0}$ subset of $2^{\omega}$, so that for some $B_{n} \subset 2^{\omega}, B_{n} \in \Pi_{1+\xi_{n}}^{0}, B=\bigcap_{n} B_{n}^{\vee}$. By the hypothesis, let $\sigma_{n}$ be a winning strategy for player II in $G_{w}\left(B_{n}, A_{n}\right)$. We define a strategy for II in $G_{w}(B, A)$ : If at stage $k$ I has played $u$ of length $k+1$, II considers

$$
u_{k}= \begin{cases}u & \text { on } \pi^{-1} \pi(k) \cap \operatorname{lh} u, \\ 0 & \text { elsewhere }\end{cases}
$$

and answers by $\sigma_{\pi(k)}\left(u_{k}\right)$. If in a row I has played $\varepsilon$ and II has answered $\alpha$ using this strategy, let

$$
\alpha_{k}= \begin{cases}\alpha & \text { on } \pi^{-1}(k), \\ 0 & \text { elsewhere }\end{cases}
$$

and notice that $\left(\varepsilon, \alpha_{k}\right)$ is a play in $G_{w}\left(B_{k}, A_{k}\right)$ where II follows $\sigma_{k}$, hence $\varepsilon \in B_{k} \leftrightarrow$ $\alpha_{k} \in A_{k}$. But by the independence property $\alpha_{k} \in A_{k} \leftrightarrow \alpha \in A_{k}$, hence $\varepsilon \in B \leftrightarrow \alpha$ $\in A$ and the strategy is winning for II in $G_{u}(B, A)$.

Proof of Theorem 2. We do it by induction on $\eta$. For $\eta=1$, we take for $\underline{R}^{1}$ the basic ramification $\underline{R}$ of Lemma 3. One checks that $\rho_{0}^{1}=\rho_{0}$ is an independent 1 -function from its very definition. At a successor step $\eta=\xi+1$, we let $\underline{R}^{\eta}=R \circ \underline{R}^{\xi}$. This is a ramification by Lemma 4 , and $\rho^{\eta}$ and $f^{\eta}$ are clearly of class $\eta$. So we just 
need to prove that $\rho_{0}^{\eta}=\rho_{0} \circ \rho_{0}^{\xi}$ is an independent $\eta$-function. By definition

$$
\rho_{0}^{\eta}(\varepsilon)(k)=1 \leftrightarrow \rho_{0}\left(\rho_{0}^{\xi}(\varepsilon)\right)(k)=1 \leftrightarrow \forall i \rho_{0}^{\xi}(\varepsilon)(\langle k, i\rangle)=0
$$

By the inductive hypothesis, there is some $\pi_{\xi}: \omega \rightarrow \omega$ such that $\rho_{0}^{\xi}(\varepsilon)(n)$ depends only on $\varepsilon \uparrow \pi_{\xi}^{-1}(n)$. Applying Lemma 7 to $A_{i}=\left\{\varepsilon \mid \rho_{0}^{\xi}(\varepsilon)(\langle k, i\rangle)=1\right\}$ and $\pi_{\xi}^{k}: \omega \rightarrow \omega$ defined by $\pi_{\xi}^{k}(i)=\pi_{\xi}(\langle k, i\rangle)$, the set $A^{k}=\left\{\varepsilon \mid \rho_{0}^{\eta}(\varepsilon)(k)=1\right\}$ is strategically complete $\Pi_{1+\xi}^{0}$. And if $\pi_{\eta}: \omega \rightarrow \omega$ is defined by $\pi_{\eta}(n)=\left(\pi_{\xi}(n)\right)_{0}, \rho_{0}^{\eta}(\varepsilon(k))$ depends only on $\varepsilon \uparrow \pi_{\eta}^{-1}(k)$, so $\rho_{0}^{\eta}$ is an independent $\eta$-function. And finally $H_{1+\eta}=\left(\rho_{0}^{\eta}\right)^{-1}(\underline{0})$ is strategically complete $\Pi_{1+\eta}^{0}$ by Lemma 7 again. Suppose finally that the construction has been performed up to some limit $\eta$, and let $\left(\xi_{n}\right)$ be a sequence of ordinals with $\eta=\sum_{n} \xi_{n}$; let $\eta_{n}=\sum_{0}^{n} \xi_{k}$, and apply Lemma 6 to $\underline{R}_{i}=\underline{R}^{\xi_{i}}$. This gives a sequence $\underline{R}^{i, \omega}$, and we define $\underline{R}^{\eta}=\underline{R}^{0, \omega}$. By the definition of the $\underline{R}^{i, \omega}$ 's, one easily checks that $\rho^{\eta}$ and $f^{\eta}$ are of class $\eta$. Now $\rho_{0}^{\eta}(\varepsilon)=\lim _{n} \rho_{0}^{(0, n)}(\varepsilon)$, in fact $\rho_{0}^{\eta}(\varepsilon)(k)=$ $\rho_{0}^{(0, k+1)}(\varepsilon)(k)$. By the inductive hypothesis, one checks that $\left\{\varepsilon \mid \rho_{0}^{(0, k+1)}(\varepsilon)(k)=1\right\}$ is strategically complete $\Pi_{1+\eta_{k}}^{0}$, and that $\rho_{0}^{\eta}$ is independent. Finally $H_{1+\eta}$ is strategically complete $\Pi_{1+\eta}^{0}$ by Lemma 7 again.

Notice that in the above proof, the $\underline{R}^{\eta}$ 's we constructed are almost unique-up to some choice, for limit $\eta$, of a sequence $\left(\xi_{k}\right)$ with $\eta=\Sigma_{k} \xi_{k}$. We shall very freely refer to these ramifications as the ramification $\underline{R}^{\eta}$ of order $\eta$-the choice of the fundamental sequences at limit steps being usually "the best one" in each concrete situation.

4. The games $J_{\xi}$. The property of Wadge in Polish spaces. We now come back to the proof of Theorem 1.1. As before, we fix a pair of disjoint $\Sigma_{1}^{1}$ sets $A_{0}, A_{1}$ in $2^{\omega}$, with corresponding trees $T_{0}, T_{1}$, and let $\xi=1+\eta$ be some countable ordinal $\geqslant 1$. Let $\underline{R}^{n}=\left(r^{\eta}, \rho^{\eta}, f^{\eta}\right)$ be the ramification given by Theorem 3.2. We let $H_{\xi}=$ $\left(\rho_{0}^{\eta}\right)^{-1}(\underline{0})$. By $3.2, H_{\xi}$ is a strategically complete $\Pi_{\xi}^{0}$ set.

We define the game $J_{\xi}\left(A_{0}, A_{1}\right)$ as follows: player I produces $\varepsilon \in 2^{\omega}$, player II produces $(\alpha, \beta) \in 2^{\omega} \times \omega^{\omega}$. We say that $(\varepsilon, \alpha, \beta)$ is a win for II in $J_{\xi}\left(A_{0}, A_{1}\right)$ if $(\varepsilon, \beta)$ is a win for II in the game $R^{\eta}\left(J_{1}\left(A_{0}, A_{1}\right) \uparrow_{\alpha}\right)$ (where of course $J_{1}\left(A_{0}, A_{1}\right)$ is the game defined in \$2.2). We now show that $H_{\xi}$ and $J_{\xi}\left(A_{0}, A_{1}\right)$ satisfy the conclusions of Theorem 1.1, for $\Gamma=\Pi_{\xi}^{0}$.

First of all, $J_{\xi}\left(A_{0}, A_{1}\right)$ is closed: The point is that ramifications do not increase the length of sequences, hence in order to verify that $\left(\varepsilon \uparrow_{k}, \beta \uparrow_{k}\right)$ is a legal position in $J_{\xi}\left(A_{0}, A_{1}\right) \uparrow_{\alpha}=R^{\eta}\left(J_{1}\left(A_{0}, A_{1}\right) \uparrow_{\alpha}\right)$, we only need to know $\alpha \uparrow_{k}$. Suppose now that II has a winning strategy $\tau$ in $J_{\xi}\left(A_{0}, A_{1}\right)$, with associated $\tau^{*}$ in $G_{w}^{*}\left(H_{\xi} ; A_{0}, A_{1}\right)$. To each $\varepsilon \in 2^{\omega}, \tau$ associates some $\beta$ such that $(\varepsilon, \beta)$ is a legal play for II in $R^{\eta}\left(J_{1}\left(A_{0}, A_{1}\right) \uparrow_{\tau^{*}(\varepsilon)}\right)$, and by the projection $\rho^{\eta}$, we get a play $\left(\rho_{0}^{\eta}(\varepsilon), \beta \circ \rho_{1}^{\eta}(\varepsilon)\right)$ which is legal for II in $J_{1}\left(A_{0}, A_{1}\right) \uparrow{ }_{\tau^{*}(\varepsilon)}$. Using Lemma 2.1, we get that if $\rho_{0}^{\eta}(\varepsilon)=\underline{0}$, i.e. if $\varepsilon \in H_{\xi}$, then $\tau^{*}(\varepsilon) \in A_{0}$; and if $\rho_{0}^{\eta}(\varepsilon) \neq \underline{0}$, i.e. $\varepsilon \notin H_{\xi}$, then $\tau^{*}(\varepsilon) \in A_{1}$. This means $\tau^{*}$ is winning for player II in $G_{w}^{*}\left(H_{\xi} ; A_{0}, A_{1}\right)$. Suppose finally that I has a winning strategy $\sigma$ in $J_{\xi}\left(A_{0}, A_{1}\right)$; for each $\alpha \in 2^{\omega}$, let $\sigma_{\alpha}$ be the corresponding winning strategy for $\mathrm{I}$ in $J_{\xi}\left(A_{0}, A_{1}\right) \uparrow_{\alpha}=R^{\eta}\left(J_{1}\left(A_{0}, A_{1}\right) \uparrow_{\alpha}\right)$. Then for each $\eta$ the strategy $\sigma_{\alpha}^{*}=F^{\eta}\left(J_{1}\left(A_{0}, A_{1}\right) \uparrow_{\alpha}, \sigma_{\alpha}\right)$ is winning for I in $J_{1}\left(A_{0}, A_{1}\right) \uparrow_{\alpha}$. Moreover, the function $\sigma^{*}: 2^{\omega} \rightarrow \Sigma$ defined by $\sigma^{*}(\alpha)=\sigma_{\alpha}^{*}$ is clearly of class $\eta$. So 
if we set $C_{\sigma}=\left\{\alpha \in 2^{\omega} \mid\left(\alpha, \sigma_{\alpha}^{*}\right) \in C\right\}$ where $C$ is the open set defined in Lemma 2.1 , we get by this lemma that $C_{\sigma}$ is a $\Sigma_{\xi}^{0}$ set which separates $A_{0}$ from $A_{1}$. This finishes the proof for $\Gamma=\Pi_{\xi}^{0}$ (hence $\Gamma=\Sigma_{\xi}^{0}$ too). It remains to look at $\Gamma=D_{\xi}\left(\Sigma_{\xi}^{0}\right)$. We again let $\xi=\eta+1$, and define the game $J_{\xi, \xi}\left(A_{0}, A_{1}\right)$ by the equality

$$
J_{\xi, \zeta}\left(A_{0}, A_{1}\right) \uparrow_{\alpha}=R^{\eta}\left(J_{1, \zeta}\left(A_{0}, A_{1}\right) \uparrow_{\alpha}\right)
$$

where $J_{1 . \zeta}$ refers to the game of $\S 2$. The proof that this game works is entirely analogous to the preceding proof, this time using Lemma 2.4 instead of Lemma 2.1. The only point to check for finishing the proof of Theorem 1.1. is that if we set $H_{\xi, \zeta}=\left(\rho_{0}^{\eta}\right)^{-1}\left(H_{1, \zeta}\right)$ and $G_{\xi, \zeta}=\check{H}_{\xi, \zeta}$, the set $G_{\xi, \zeta}$ is a strategically complete $D_{\zeta}\left(\Sigma_{\xi}^{0}\right)$ set.

To see this, we come back to the definition of $G_{1, \xi}$. If $\psi=\left(\psi_{0}, \psi_{1}\right)$ is the bijection between $\omega$ and $\zeta \times \omega$ used to define $G_{1, \zeta}$, one checks that we have $G_{\xi, \zeta}=$ $D_{\zeta}\left(\left(G_{\zeta^{\prime}}\right)_{\zeta^{\prime}<\zeta}\right)$, where for $\zeta^{\prime}<\zeta G_{\zeta^{\prime}}=\left\{\alpha \in 2^{\omega} \mid \exists i\left(\psi_{0}(i) \leqslant \zeta^{\prime}\right.\right.$ and $\left.\left.\rho_{0}^{\eta}(\alpha)(i)=1\right)\right\}$. Define

$$
G_{\zeta^{\prime}}^{\prime}=\left\{\alpha \in 2^{\omega} \mid \exists i\left(\psi_{0}(i)=\zeta^{\prime} \text { and } \rho_{0}^{\eta}(\alpha)(i)=1\right)\right\}
$$

By Lemma 3.7 , the sets $G_{\zeta^{\prime}}^{\prime}$ are strategically complete $\Sigma_{\xi}^{0}$ sets; moreover if $A_{\zeta^{\prime}}=$ $\pi_{\eta}^{-1}\left(\psi_{0}^{-1}\left(\zeta^{\prime}\right)\right)$, where $\pi_{\eta}$ witnesses that $\rho_{0}^{\eta}$ is independent, the sets $\left(A_{\zeta^{\prime}}\right)_{\zeta^{\prime}<\zeta}$ are mutually disjoint, and the fact that $\alpha \in G_{\zeta^{\prime}}^{\prime}$ depends only on $\alpha \uparrow A_{\zeta^{\prime}}$. Suppose now $B=D_{\zeta}\left(\left(B_{\zeta^{\prime}}\right)\right)$ is another $D_{\zeta}\left(\Sigma_{\xi}^{0}\right)$ set, with $\left(B_{\zeta^{\prime}}\right)$ an increasing family of $\Sigma_{\xi}^{0}$ sets in $2^{\omega}$. Pick for each $\zeta^{\prime}<\zeta$ some $\sigma_{\zeta^{\prime}}$ which is winning for II in $G_{w}\left(B_{\zeta^{\prime}}, G_{\zeta^{\prime}}^{\prime}\right)$. Define a strategy for II in $G_{w}\left(B, G_{\xi, \zeta}\right)$ as follows: If I plays at stage $k$ some $u$ of length $k+1$, II answers by $\sigma_{\zeta^{\prime}}(u)$, where $\zeta^{\prime}=\psi_{0}\left(\pi_{\eta}(k)\right)$. If $\varepsilon$ and $\alpha$ have been played this way, let $\alpha_{\zeta^{\prime}}=\overline{\sigma_{\zeta^{\prime}}}(\varepsilon)$, for each $\zeta^{\prime}<\zeta$. As $\sigma_{\zeta^{\prime}}$ is winning for II in $G_{w}\left(B_{\zeta^{\prime}}, G_{\zeta^{\prime}}^{\prime}\right), \varepsilon \in B_{\zeta^{\prime}} \leftrightarrow$ $\alpha_{\zeta^{\prime}} \in G_{\zeta^{\prime}}^{\prime}$. Now $\alpha_{\zeta^{\prime}}$ and $\alpha$ coincide on $A_{\zeta^{\prime}}$, hence $\alpha_{\zeta^{\prime}} \in G_{\zeta^{\prime}}^{\prime} \leftrightarrow \alpha \in G_{\zeta^{\prime}}^{\prime}$. So suppose $\varepsilon \in B_{\zeta^{\prime}}$. Then $\alpha \in G_{\zeta^{\prime}}^{\prime}$ and a fortiori $\alpha \in G_{\zeta^{\prime}}$. And if $\varepsilon \notin B_{\zeta^{\prime}}$, then a fortiori for all $\zeta^{\prime \prime} \leqslant \zeta^{\prime} \varepsilon \notin B_{\zeta^{\prime \prime}}$, hence for all $\zeta^{\prime \prime} \leqslant \zeta^{\prime} \alpha \notin G_{\zeta^{\prime \prime}}^{\prime \prime}$, and $\alpha \notin G_{\zeta^{\prime}}$. This shows that the strategy is winning for II, hence $G_{\xi, \zeta}$ is strategically complete $D_{\zeta}\left(\Sigma_{\xi}^{0}\right)$, and the proof of Theorem 1.1 is complete.

If we want to compare the descriptive complexity of sets in arbitrary Polish space $E$ and $F$, the ordering $\leqslant$, given by Wadge's games is no longer adequate: there seems to be no interesting extension of it to this wider frame-except for spaces like $\omega^{\omega}$. A more relevant ordering, usually called the Wadge ordering $\leqslant_{w}$ (see [W 1$]$ ), uses reduction by continuous functions: If $A \subseteq E$ and $B \subseteq F$ are two sets, we say that $B$ reduces $A\left(A \leqslant{ }_{w} B\right)$, if for some continuous map $\varphi: E \rightarrow F \varphi^{-1}(B)=A$. Similarly if $B_{0}, B_{1}$ are disjoint subsets of $F$, the pair $\left(B_{0}, B_{1}\right)$ reduces $A$ if for some continuous map $\varphi: E \rightarrow B_{0} \cup B_{1}, \varphi^{-1}\left(B_{0}\right)=A$. Note that these notions are extrinsic, i.e. depend heavily on the space $E$ in which $A$ sits (This notion might well not be the correct one for arbitrary Polish spaces, as it clearly depends on how many continuous functions from $E$ into $F$ exist, hence depends on the dimensions of $E$ and $F$. Here we shall work with $E=2^{\omega}$, for which this problem disappears.) 
Let $\Gamma$ be some class of subsets of $2^{\omega}$. We say that a subset $A$ of a Polish space $E$ reduces $\Gamma$ if for any $B \subseteq 2^{\omega}, B \in \Gamma, A$ reduces $B$ (and similarly for a disjoint pair $\left(A_{0}, A_{1}\right)$ ). The dual notion is that of separation: We say that $\Gamma$ separates a disjoint pair $\left(A_{0}, A_{1}\right)$ in some Polish space $E$ if for some $C \in \Gamma, C$ separates $A_{0}$ from $A_{1}$.

With this terminology, an immediate consequence of Theorem 1.1 is the following

THEOREM 1. Let $E=2^{\omega}$, and $\Gamma$ be either $\Sigma_{\xi}^{0}$ or $D_{\zeta}\left(\Sigma_{\xi}^{0}\right)$, with dual class $\check{\Gamma}$. If $\left(A_{0}, A_{1}\right)$ is any pair of disjoint $\Sigma_{1}^{1}$ subsets of $E$, either $\left(A_{0}, A_{1}\right)$ reduces $\Gamma$ or $\check{\Gamma}$ separates $\left(A_{0}, A_{1}\right)$.

We will now prove the extension of this result to arbitrary Polish spaces. The key tool is a transfer lemma, due to Saint Raymond [SR 3], and independently reproved by Kunen and Miller [K-M].

THEOREM (SAINT RAYMOND). Let $E, F$ be two compact metrizable spaces, and $\pi a$ continuous surjection: $E \rightarrow F$. Suppose $P$ is some Polish space, and $f: E \rightarrow P$ a first class function. Then there exists a first class section $s: F \rightarrow E$ of $\pi$ (i.e. such that $\pi \circ s(x)=x$ for all $x \in F)$, satisfying $f \circ s: F \rightarrow P$ is also a first class function.

Let us introduce some more terminology. For a class $\Gamma$ (of sets in Polish spaces), let us say that $\Gamma$ has the property of Wadge if for any pair of disjoint $\Sigma_{1}^{1}$ sets $A_{0}, A_{1}$ (in some Polish space $E$ ) either $\left(A_{0}, A_{1}\right)$ reduces $\Gamma$ or $\check{\Gamma}$ separates $\left(A_{0}, A_{1}\right)$.

To each set $D \subseteq 2^{\omega}$, associate an operation (also called $D$ ), the Hausdorff operation with basis $D$, on sequences of sets by setting, for $\left(A_{n}\right)_{n \in \omega}$ subsets of some set $X, D\left(\left(A_{n}\right)\right)=\left\{x \in X \mid\left\{n \in \omega \mid x \in A_{n}\right\} \in D\right\}$. We say that a class $\Gamma$ is a $\Sigma_{2}^{0}$-generated Hausdorff class if there is a basis $D \subseteq 2^{\omega}$ such that in any Polish space $E, \Gamma$ consists of the sets $D\left(\left(A_{n}\right)\right)$ for $\left(A_{n}\right) \Sigma_{2}^{0}$ subsets of $E$. Clearly most classes of descriptive set theory (among which the $\Sigma_{\xi}^{0}$ and $D_{\eta}\left(\Sigma_{\xi}^{0}\right)$ classes, for $\xi \geqslant 2$ ) are $\Sigma_{2}^{0}$-generated Hausdorff classes.

Corollary 2 (The TRANSFer lemma). Let $E, F$ be two compact metrizable spaces, $\pi: E \rightarrow F$ a continuous surjection, and $\Gamma$ a $\Sigma_{2}^{0}$-generated Hausdorff class. Let $A_{0}, A_{1}$ be two disjoint subsets of $F$. If the class $\Gamma$ separates the pair $\left(\pi^{-1}\left(A_{0}\right), \pi^{-1}\left(A_{1}\right)\right)$, it also separates $\left(A_{0}, A_{1}\right)$. In particular, if $A \subseteq F$ is such that $\pi^{-1}(A) \in \Gamma$, the set $A$ is in $\Gamma$.

Proof. Let $C \subseteq E, C$ in $\Gamma$, separate $\pi^{-1}\left(A_{0}\right)$ from $\pi^{-1}\left(A_{1}\right)$, and write $C=$ $D\left(\left(C_{n}\right)\right)$, where $D$ is the basis for $\Gamma$ and $C_{n}, n \in \omega$, are $\Sigma_{2}^{0}$ sets in $E$. For each $n$, let $f_{n}: E \rightarrow[0,1]$ be a first class function so that $C_{n}=\left\{x \in E \mid f_{n}(x)>0\right\}$, and let $f$ : $E \rightarrow[0,1]^{\omega}$ be $\Pi_{n} f_{n}$. Apply Saint Raymond's theorem to $\pi$ and $f:$ There is a section $s$ of $\pi$ such that $s$ and $f \circ s$ are of first class. Let $B_{n}=\left\{x \in F \mid f_{n} \circ s(x)>0\right\}$, and $B=D\left(\left(B_{n}\right)\right)$. Each $B_{n}$ is clearly $\Sigma_{2}^{0}$, hence $B \in \Gamma$. And one easily checks that $B$ separates $A_{0}$ from $A_{1}$.

THEOREM 3. For each $\xi \geqslant 2$, the classes $\Sigma_{\xi}^{0}$ and $D_{\eta}\left(\Sigma_{\xi}^{0}\right)$ have the property of Wadge. 
Proof. Let $E$ be a Polish space, $A_{0}, A_{1}$ two disjoint $\Sigma_{1}^{1}$ subsets of $E$. Extend $E$ to some compact metrizable $\hat{E}$, and choose a continuous surjection $\pi: 2^{\omega} \rightarrow \hat{E}$. Let $B_{0}=\pi^{-1}\left(A_{0}\right), B_{1}=\pi^{-1}\left(A_{1}\right)$. Using Theorem 1, either $\left(B_{0}, B_{1}\right)$ reduces $\Gamma$, in which case $\left(A_{0}, A_{1}\right)$ too-by composing with $\pi$-or $\check{\Gamma}$ separates $\left(B_{0}, B_{1}\right)$ and by Corollary $2 \check{\Gamma}$ also separates $\left(A_{0}, A_{1}\right)$ (in $\hat{E}$, hence in $E$ ).

5. Hurewicz-type theorems. In this section, we generalize the theorem of Hurewicz stated in the introduction, asserting that two disjoint analytic sets $A_{0}$ and $A_{1}$ in a Polish space are not separable by a $\Sigma_{2}^{0}$ set if and only if there exists a homeomorphism $\varphi$ from $2^{\omega}$ onto a (compact) subset $K$ of $A_{0} \cup A_{1}$ such that $\varphi\left(\mathbf{P}_{\infty}\right)=A_{0} \cap K$ and $\varphi\left(\mathbf{P}_{f}\right)=A_{1} \cap K$. In other words, $\Sigma_{2}^{0}$ does not separate $\left(A_{0}, A_{1}\right)$ iff there is a continuous one-to-one map reducing $\mathbf{P}_{\infty}$ to the pair $\left(A_{0}, A_{1}\right)$.

By analogy with the definitions in the preceding section, let us say that a pair ( $\left.A_{0}, A_{1}\right) \quad H$-reduces a set $B \subseteq 2^{\omega}$ if for some continuous one-to-one map $\varphi$ : $2^{\omega} \rightarrow A_{0} \cup A_{1}, B=\varphi^{-1}\left(A_{0}\right)$. (If $A_{1}=A_{0}^{\vee}$, we just say that $A_{0} H$-reduces $B$.) And given a class $\Gamma$ of subsets of $2^{\omega}$, we say $\left(A_{0}, A_{1}\right) H$-reduces $\Gamma$ (resp. $A_{0} H$-reduces $\Gamma$ ) if it reduces all sets $B \in \Gamma, B \subseteq 2^{\omega}$.

We say that a set $A \in \Gamma$ is an $H$-complete $\Gamma$ set if $A H$-reduces $\Gamma$. The existence of such sets is given by the next lemma.

Lemma 1. Let $\Gamma$ be some class, and let $A \in \Gamma, A \subseteq 2^{\omega}$ be complete, i.e. A reduces $\Gamma$. Then the set $A \times 2^{\omega} \subseteq 2^{\omega} \times 2^{\omega} \simeq 2^{\omega}$ is an $H$-complete $\Gamma$ set.

Proof. Let $B$ be a $\Gamma$ subset of $2^{\omega}$, and $\varphi: 2^{\omega} \rightarrow 2^{\omega}$ reduce $B$ to $A$. Then $\psi: 2^{\omega} \rightarrow 2^{\omega} \times 2^{\omega}$ defined by $\psi(\alpha)=(\varphi(\alpha), \alpha)$ is one-to-one and reduces $B$ to $A \times 2^{\omega}$.

In some sense, $H$-complete sets in $\Gamma$ are maximal. In particular, a pair $\left(A_{0}, A_{1}\right)$ $H$-reduces a class $\Gamma$ as soon as it $H$-reduces some $H$-complete set in $\Gamma$. We now introduce the "minimal" sets in $\Gamma$.

Definition 2. A set $X \subseteq 2^{\omega}$ is a Hurewicz test for a class $\Gamma$ if

(i) $X \in \Gamma$ and $X \notin \check{\Gamma}$,

(ii) For any Borel set $B$ in a Polish space $E, B$ is not in the dual class $\check{\Gamma}$ if and only if $B H$-reduces $X$.

We denote by $\operatorname{Hur}(\Gamma)$ the set of Hurewicz tests for $\Gamma$.

The next lemma explains why such sets $X$ are called Hurewicz tests. Recall that $\Gamma$ has the property of Wadge if for any pair of analytic disjoint sets $\left(A_{0}, A_{1}\right)$ in some Polish space $E$, either $\left(A_{0}, A_{1}\right)$ reduces $\Gamma$ or $\check{\Gamma}$ separates $\left(A_{0}, A_{1}\right)$.

Lemma 3. Let $\Gamma$ be a class with the property of Wadge, and $X$ belong to $\operatorname{Hur}(\Gamma)$. Then for any Polish space $E$ and any pair $\left(A_{0}, A_{1}\right)$ of disjoint analytic subsets of $E$, the class $\check{\Gamma}$ does not separate $\left(A_{0}, A_{1}\right)$ if and only if $\left(A_{0}, A_{1}\right)$ H-reduces $X$.

Proof. If $\left(A_{0}, A_{1}\right) H$-reduces $X$ but $\check{\Gamma}$ separates $\left(A_{0}, A_{1}\right)$, then clearly $X \in \check{\Gamma}$, which is impossible.

Conversely if $\check{\Gamma}$ does not separate $\left(A_{0}, A_{1}\right)$, then $\left(A_{0}, A_{1}\right)$ reduces $X$ by the property of Wadge, i.e. for some continuous $\varphi: 2^{\omega} \rightarrow A_{0} \cup A_{1}, \varphi^{-1}\left(A_{0}\right)=X$. The sets $B_{0}=\varphi(X)$ and $B_{1}=\varphi(\check{X})$ are $\Sigma_{1}^{1}$ and disjoint, with union the compact set $\varphi\left(2^{\omega}\right)$. hence are Borel. 
And $B_{0}$ cannot be in $\check{\Gamma}$, otherwise $X=\varphi^{-1}\left(B_{0}\right)$ would be in $\check{\Gamma}$ too. So as $X$ is a Hurewicz test, $B_{0} H$-reduces $X$ and a fortiori $\left(A_{0}, A_{1}\right) H$-reduces $X$.

The notions of Hurewicz test and of $H$-complete set in a class $\Gamma$ usually do not coincide-for example in the basic case of $\Sigma_{2}^{0}$ studied by Hurewicz. Note however that if there exists an $H$-complete set in $\operatorname{Hur}(\Gamma)$, then the notions of $H$-complete, complete, Hurewicz test, and $\Gamma \backslash \check{\Gamma}$ sets all coincide.

The main result of this section is the existence of Hurewicz tests for all classes $\Sigma_{\xi}^{0}$ and $D_{\eta}\left(\Sigma_{\xi}^{0}\right)$, for $\xi \geqslant 2$. For the classes $D_{\eta}\left(\Sigma_{1}^{0}\right)$, no Hurewicz test can be found in $2^{\omega}$ for cardinality reasons. Nevertheless it is possible (and easy) to replace the space $2^{\omega}$ by some suitable countable compact space $K_{\eta}$ and construct a subset $X_{\eta}$ of $K_{\eta}$ which serves as Hurewicz test for the class $D_{\eta}\left(\Sigma_{1}^{0}\right)$.

THEOREM 4. Let $\Gamma$ be a class closed under unions with $\Sigma_{2}^{0}$ sets and intersections with $\Pi_{2}^{0}$ sets. Then every pair $\left(A_{0}, A_{1}\right)$ which reduces $\Gamma$ also $H$-reduces $\Gamma$.

We are grateful to A. S. Kechris for pointing out to us, after we obtained this result and the corollary stated below, that a similar result had been obtained earlier by J. R. Steel [S]. In fact, Lemma 3 of [S] asserts that (with our terminology) every complete $\Gamma$ set in $2^{\omega} H$-reduces any $\Gamma$ set in $2^{\omega}$, provided $\Gamma$ is "reasonably closed". (The proof is quite similar to ours, and is referred to by Steel as a "trick due to Harrington".) The closure condition of Steel is rather technical and looks ad hoc. However, it can be shown that it corresponds to closure by intersections with $\Pi_{2}^{0}$ and unions with $\Sigma_{2}^{0}$ sets, plus closure under homeomorphisms (i.e. if $A \in \Gamma$ and $B$ is homeomorphic with $A, B \in \Gamma$ ). So our Theorem 4 is slightly more general (it can indeed be applied to more Wadge classes), and also works for arbitrary Polish spaces. It is why we include it here.

We obtain Theorem 4 as a particular case of Theorem 7 below, which is necessary for the classes $D_{n}\left(\Sigma_{2}^{0}\right)$.

COROllary 5. Let $\Gamma$ be a class which is non selfdual $(\Gamma \neq \check{\Gamma})$, has the Wadge property and is closed under unions with $\Sigma_{2}^{0}$ sets and intersections with $\Pi_{2}^{0}$ sets. Then

(i) $\operatorname{Hur}(\Gamma)=\Gamma \backslash \check{\Gamma}$.

(ii) For any pair of disjoint analytic sets in some Polish space E, the class $\check{\Gamma}$ does not separate $\left(A_{0}, A_{1}\right)$ iff $\left(A_{0}, A_{1}\right) H$-reduces $\Gamma$.

COROllaRy 6. Let $\Gamma$ be one of the classes $\Sigma_{\xi}^{0}$ or $D_{\eta}\left(\Sigma_{\xi}^{0}\right)$, for $\xi \geqslant 3$, or $\xi=2$ and $\eta \geqslant \omega$. Then conclusions (i) and (ii) of Corollary 5 hold.

The preceding corollaries are not true for $\Sigma_{2}^{0}: H$-complete $\Sigma_{2}^{0}$ sets must have cardinality the continuum, and Hurewicz tests for $\Sigma_{2}^{0}$ are at most countable. To deal with $\Sigma_{2}^{0}$ (and the classes $D_{n}\left(\Sigma_{2}^{0}\right)$ ), we generalize Theorem 4 in the following

THEOREM 7. Let $\Gamma_{0}$ be a class closed under intersections with $\Pi_{2}^{0}$ sets, and $\Gamma$ the class of unions of sets in $\Gamma_{0}$ with $\Sigma_{2}^{0}$ sets. Let $B_{0}$, D be subsets of $2^{\omega}$ with $B_{0} \in \Gamma_{0}$ and $D$ at most countable, and let $B=B_{0} \cup D$. Then if a pair $\left(A_{0}, A_{1}\right)$ reduces the class $\Gamma$, ( $\left.A_{0}, A_{1}\right) H$-reduces $B$.

Theorem 4 easily follows from Theorem 7 , for $\Gamma=\Gamma_{0}$ and $D=\varnothing$. 
Corollary 8. Let $\Gamma$ be the class $D_{n}\left(\Sigma_{2}^{0}\right)$, for $n<\omega$. Let $\left(B_{k}\right)_{k<n}$ be some increasing sequence of $\Sigma_{2}^{0}$ sets in $2^{\omega}$, with $B_{0}$ at most countable, and let $B=D_{n}\left(\left(B_{k}\right)\right)$. If $\left(A_{0}, A_{1}\right)$ is a pair of disjoint $\Sigma_{1}^{1}$ sets in some Polish space and $\check{\Gamma}$ does not separate ( $\left.A_{0}, A_{1}\right)$, then $\left(A_{0}, A_{1}\right) H$-reduces $B$.

Proof. Let $\Gamma_{0}$ be the class of all $D_{n}\left(\left(A_{k}\right)\right)$, with $A_{k} \in \Sigma_{2}^{0}$ and $A_{0}=\varnothing$. If $n$ is odd, $\Gamma_{0}$ is closed under intersections with $\Pi_{2}^{0}$ sets and $\Gamma$ is the class of unions of $\Gamma_{0}$ sets and $\Sigma_{2}^{0}$ sets. Since $\Gamma$ has the Wadge property, we can apply Theorem 7 to get the result. The case of even $n$ is similar, by working with $\check{\Gamma}_{0}$ and $\check{\Gamma}$.

COROllary 9. For each $n<\omega$, define a sequence $B_{k}, k<n$, by

$$
B_{k}=\left\{\left(\alpha_{0}, \ldots, \alpha_{n-1}\right) \in\left(2^{\omega}\right)^{n} \mid \forall j \leqslant k \alpha_{j} \in \mathbf{P}_{f}\right\},
$$

and let $B=D_{n}\left(B_{k}\right)$. Then $B \in \operatorname{Hur}\left(D_{n}\left(\Sigma_{2}^{0}\right)\right)$.

Proof. By Corollary 8 , we just need to show that $B$ is a complete $D_{n}\left(\Sigma_{2}^{0}\right)$ set, as $B_{0}=\mathbf{P}_{f}^{n}$ is countable. But using that $\mathbf{P}_{f}$ is complete $\Sigma_{2}^{0}$, this is immediate.

We now turn to the proof of Theorem 7. We need the following improvement of Baire's category theorem.

LeMma 10. Let $E$ be a complete metric space, $\left(A_{s}\right)_{s \in \omega^{<\omega}}$ a Suslin scheme of closed subsets of $E$, with $H=\bigcup_{\alpha \in \omega^{\omega}} \bigcap_{n} A_{\alpha \uparrow n}$ its kernel, and $\left(U_{n}\right)_{n \in \omega}$ a sequence of open subsets of $E$, satisfying

(i) $A_{\varnothing}=E$.

(ii) For all $s \in \omega^{<\omega}, A_{s}={\overline{\bigcup_{m} A_{s^{\wedge} m}}}_{\text {. }}$

(iii) For all $s \in \omega^{<\omega}, n \in \omega, A_{s}=\overline{A_{s} \cap U_{n}}$.

Then the set $H \cap \cap_{n} U_{n}$ is dense in $E$. (If for all $s A_{s}=E$, this is just Baire's theorem.)

Proof. By replacing $E$ by a nonempty open subset of it, it is enough to prove $H \cap \bigcap_{n} U_{n} \neq \varnothing \cdot$ (for $E \neq \varnothing$ !). We construct inductively open balls $B_{k}$ in $E$ with center $x_{k}$ and radius $r_{k}$, and integers $m_{k}$ so that for all $k$

(a) $B_{k} \subset U_{k}$ and $r_{k}<1 / k$.

(b) $\bar{B}_{k+1} \subset B_{k}$.

(c) $x_{k} \in A_{\left(m_{0}, \ldots, m_{k-1}\right)}$.

For this, choose $x_{0}$ in $U_{0}$, and $r_{0}$ so that $B_{0} \subset U_{0}$. If $x_{k}, r_{k}, m_{i}, i<k$ are defined, we can choose by (ii) $m_{k}$ so that $B_{k} \cap A_{\left(m_{0}, \ldots, m_{k}\right)} \neq \varnothing$, then $x_{k+1}$ in $U_{k} \cap B_{k} \cap$ $A_{\left(m_{0}, \ldots, m_{k}\right)}$, and $r_{k+1}<1 /(k+1)$ such that $\bar{B}_{k+1} \subset U_{k+1} \cap B_{k}$. The sequence $\left(x_{k}\right)_{k \in \omega}$ is a Cauchy sequence in $E$, and clearly its limit $x$ is in $H \cap \cap_{n} U_{n}$.

Proof of Theorem 7. For $\alpha \in 2^{\omega}$, let $\alpha^{*}$ be defined by $\alpha^{*}(n)=1-\alpha(n)$, and denote accordingly $\mathbf{P}_{f}^{*}=\left\{\alpha \mid \alpha^{*} \in \mathbf{P}_{f}\right\}$ and $\mathbf{P}_{\infty}^{*}=\left\{\alpha \mid \alpha^{*} \in \mathbf{P}_{\infty}\right\}$. Let $B_{0}, D, B, A_{0}$ and $A_{1}$ be as in the hypotheses of Theorem 7 . Consider the set $Z=\left(2^{\omega} \times \mathbf{P}_{f}\right) \cup$ $\left(B_{0} \times \mathbf{P}_{\infty}^{*}\right)$. By the properties of $\Gamma, Z \in \Gamma$, so there exists a continuous map $\varphi$ : $2^{\omega} \times 2^{\omega} \rightarrow\left(A_{0} \cup A_{1}\right)$ such that $Z=\varphi^{-1}\left(A_{0}\right)$. We shall prove the existence of a continuous map $g: 2^{\omega} \rightarrow 2^{\omega}$ such that $\psi_{g}=\varphi(\cdot, g(\cdot))$ is one-to-one and carries $B=B_{0} \cup D$ into $A_{0}$ and $\check{B}$ into $A_{1}$. 
Let $E$ be the space of all continuous functions: $2^{\omega} \rightarrow 2^{\omega}$, endowed with the metric of uniform convergence. Enumerate the set $\mathbf{P}_{f}$ in a sequence $\left(\alpha_{m}\right)_{m \in \omega}$ and the set $D$ in a (possibly empty or finite) sequence $\left(\delta_{k}\right)_{k<N}(0 \leqslant N \leqslant \omega)$. Set

$$
A_{m_{0} \ldots \ldots m_{k-1}}=\left\{\begin{array}{l}
\left\{g \in E \mid \forall i<k\left(i \geqslant N \text { or } g\left(\delta_{i}\right)=\alpha_{m_{i}}\right)\right\}, \\
\quad \text { if } m_{0}<m_{1}<\cdots<m_{k-1} \text { and } \\
\text { the points } \varphi\left(\delta_{i}, \alpha_{m_{i}}\right) \text { are all distinct } \\
\varnothing \quad \text { otherwise. }
\end{array}\right.
$$

Let $H=\bigcup_{\alpha \in \omega^{\omega}} \bigcap_{n} A_{\alpha \uparrow}$.

For $m \in \omega$, let $W_{m}=\left\{g \in E \mid \forall \alpha \in 2^{\omega} g(\alpha) \neq \alpha_{m}^{*}\right\}$.

Let $V_{n}=\{g \in E \mid \forall \alpha \forall \beta(d(\alpha, \beta)>1 / n \rightarrow \varphi(\alpha, g(\alpha)) \neq \varphi(\beta, g(\beta)))\}$.

Let $D_{p}=\left\{\delta_{i} \mid i<N\right.$ and $\left.i<p\right\}, K_{p, q}=\left\{\alpha \in 2^{\omega} \mid d\left(\alpha, D_{p}\right) \geqslant 1 / q\right\}$ and $U_{p, q, j}=$ $\left\{g \in E \mid \alpha_{j} \notin g\left(K_{p, q}\right)\right\}$, for $j<p$.

The sets $A_{m_{0}, \ldots, m_{k-1}}$ are closed in $E$, and the sets $W_{m}, V_{n}, U_{p, q, j}$ are open. Moreover if $g$ belongs to $H \cap \cap_{m} W_{m} \cap \cap_{n} V_{n} \cap \cap_{p, q, j} U_{p, q, j}$, then

(i) $\psi_{g}=\varphi(\cdot, g(\cdot))$ is one-to-one (since $g \in \bigcap_{n} V_{n}$ ),

(ii) $g(D) \subseteq \mathbf{P}_{f}$ (since $g \in H$ ),

(iii) $g(\check{D}) \subseteq \mathbf{P}_{\infty} \cap \mathbf{P}_{\infty}^{*}$, for if $\alpha_{m}^{*} \in g\left(2^{\omega}\right) \cap \mathbf{P}_{f}^{*}$, then $g \notin W_{m}$, and if $x \notin D$ but $g(x)=\alpha_{j}$, there is some $q$ so that $x \in K_{j+1, q}$, and $g \notin U_{j+1, q, j}$.

From this, we get that $\psi_{g}(B) \subseteq A_{0}$ and $\psi_{g}(\check{B}) \subset A_{1}$, and the proof will be complete if we can find such a $g$. And by Lemma 10, it is enough to show that $\bigcup_{m} A_{s^{\wedge} m}, A_{s} \cap W_{m}, A_{s} \cap V_{n}$ and $A_{s} \cap U_{p, q, j}$ are all dense is $A_{s}$.

So let $\left.s=\left\langle m_{0}, \ldots, m_{k-1}\right\rangle, \varepsilon\right\rangle 0$ and $g \in A_{s}$ be given. Set $k_{0}=\inf (k, N)$ and $F_{k}=\left\{\varphi\left(\delta_{i}, \alpha_{m_{i}}\right) \mid i<k_{0}\right\}$. We claim that $A_{s} \cap B(g, \varepsilon)$ intersects (a) $\cup_{m} A_{s^{\wedge} m}$, (b) $W_{m}$, (c) $V_{n}$ and (d) $U_{p, q, j}$.

(a) If $k \geqslant N, A_{s}=A_{s^{\wedge} m}$ for all $m>m_{k-1}$ and there is nothing to prove. Otherwise choose $\alpha \in \mathbf{P}_{f}^{*}$ such that $d\left(\alpha, g\left(\delta_{k}\right)\right)<\varepsilon / 2$. The image $\varphi\left(\delta_{k}, \alpha\right)$ is in $A_{1}$, and since $F_{k} \subseteq A_{0}$ one can choose $\alpha_{l}$ close enough to $\alpha$ so that $l>m_{k-1}$, $d\left(\alpha, \alpha_{l}\right)<\varepsilon / 2$ and $\varphi\left(\delta_{k}, \alpha_{l}\right) \notin F_{k}$. If then $h \in E$ agrees with $g$ on $D_{k}$, takes value $\alpha_{l}$ on $\delta_{k}$ and satisfies $d(g, h)<\varepsilon, h \in A_{s^{\wedge}} \cap B(g, \varepsilon)$.

(b) Let $m \in \omega$. Since $\alpha_{m}^{*} \notin g\left(D_{k}\right)$, there is a clopen set $V$ in $2^{\omega}$ containing $g^{-1}\left(\alpha_{m}^{*}\right)$, contained in $g^{-1}\left(B\left(\alpha_{m}^{*}, \varepsilon / 2\right)\right)$ and disjoint from $D_{k}$. Let $\alpha \in \mathbf{P}_{f} \cap$ $B\left(\alpha_{m}^{*}, \varepsilon / 2\right)$ and let $h$ be $g$ on $\check{V}$, and $\alpha$ on $V$. Then clearly $h \in A_{s} \cap W_{m} \cap B(g, \varepsilon)$.

(c) Let $n \in \omega$. Define $\gamma=\min \left\{d\left(\beta, \beta^{\prime}\right): \beta, \beta^{\prime} \in F_{k}\right.$ and $\left.\beta \neq \beta^{\prime}\right\}>0$. Choose some finite partition $\left(V_{l}\right)_{l<L}$ of $2^{\omega}$ in clopen sets of diameter less than $1 / n$, each containing at most one point of $D_{k}$, on which $g$ oscillates less than $\varepsilon / 2$ and $\varphi(\cdot, \alpha)$ oscillates less than $\gamma / 3$, for all $\alpha \in 2^{\omega}$. We may assume that $\delta_{i} \in V_{i}$, for $i<k_{0}$. So the sets $\varphi\left(V_{i} \times\left\{\alpha_{m_{l}}\right\}\right)$ are all disjoint for $i<k_{0}$. One can find a function $g^{\prime}$ taking on each $V_{l}$ a constant value $\beta_{l} \in \mathbf{P}_{f}$, such that $d\left(g, g^{\prime}\right)<\varepsilon / 2$ and $\beta_{l}=g^{\prime}\left(\delta_{i}\right)=g\left(\delta_{i}\right)$ for $i<k_{0}$. We construct a function $h$ on $2^{\omega}$, taking on each $V_{l}$ a constant value $\gamma_{l} \in \mathbf{P}_{f}$ and such that

(i) $d\left(g^{\prime}, h\right)<\varepsilon / 2$,

(ii) $\gamma_{l}=\beta_{l}=g\left(\delta_{l}\right)$ for $l<k_{0}$,

(iii) for $j<l<L, \varphi\left(V_{j} \times\left\{\gamma_{j}\right\}\right) \cap \varphi\left(V_{l} \times\left\{\gamma_{l}\right\}\right)=\varnothing$.

Clearly such an $h$ is in $A_{s} \cap B(g, \varepsilon) \cap V_{n}$. 
The $\gamma_{1}$ are given, for $l<k_{0}$. We define them inductively for $k_{0} \leqslant l<L$. Assuming (iii) for $i, j<l$, the set $T_{l}=\bigcup_{j<l}\left(V_{j} \times\left\{\gamma_{j}\right\}\right)$ is a compact subset of $A_{0}$. Let $\beta$ be in $\mathbf{P}_{f}^{*} \cap B\left(\beta_{l}, \varepsilon / 2\right)$. The compact set $S_{l}=\varphi\left(V_{l} \times\{\beta\}\right)$ is a subset of $A_{1}$, hence $S_{l} \cap T_{l}=\varnothing$, and by the uniform continuity of $\varphi$ we can find some $\gamma_{l} \in \mathbf{P}_{f}$ close enough to $\beta$ so that $d\left(\beta_{l}, \gamma_{l}\right)<\varepsilon / 2$, and $\varphi\left(V_{l} \times\left\{\gamma_{l}\right\}\right) \cap T_{l}=\varnothing$. Thus the sets $\varphi\left(V_{j} \times\left\{\gamma_{j}\right\}\right)$ are mutually disjoint for $j<l+1$, and our inductive construction is complete.

(d) Finally let $p, q, j$ be integers with $j<p$. We first show that $g^{-1}\left(\alpha_{j}\right) \cap K_{p, q} \cap$ $D_{k}=\varnothing$. If $D_{k} \subset D_{p}$, this is obvious since $D_{p} \cap K_{p, q}=\varnothing$. Otherwise we have $p \leqslant k_{0}$, and for $i<k_{0} g\left(\delta_{i}\right)=\alpha_{m_{i}}$ with $m_{0}<m_{1}<\cdots<m_{k_{0}-1}$. So $m_{i} \geqslant i$, and if $\delta_{i}$ belongs to $D_{k} \cap K_{p, q} \subset D_{k} \backslash D_{p}, m_{i} \geqslant i \geqslant p>j$ holds. So $g\left(\delta_{i}\right)=\alpha_{m_{i}} \neq \alpha_{j}$.

Let then $V$ be some clopen set in $2^{\omega}$ containing $g^{-1}\left(\alpha_{j}\right) \cap K_{p, q}$, contained in $g^{-1}\left(B\left(\alpha_{j}, \varepsilon / 2\right)\right)$ and disjoint from $D_{k}$. Choose some $\alpha \in \mathbf{P}_{\infty} \cap B\left(\alpha_{j}, \varepsilon / 2\right)$, and define $h$ to be $g$ on $\check{V}$ and the constant $\alpha$ on $V$. Then clearly $h \in A_{s} \cap U_{p, q, j} \cap$ $B(g, \varepsilon)$. This finishes the proof of Theorem 7 .

Notice that is order to derive Theorem 4 from Theorem 7 , one only uses $D=\varnothing$, and in this case $A_{s}=E$ for all $s \in \omega^{<\omega}$ and the refinement of Baire's category theorem is not necessary.

\section{Parametrized and effective results.}

6.1. Borel sets with sections of given class. As we said in the introduction, the proof of Hurewicz' theorem on $\Sigma_{2}^{0}$ sets, which is a paradigm for our results in the preceding sections, was the starting point for a result of Saint Raymond [SR 1] asserting that Borel sets with $\Sigma_{2}^{0}$ sections are countable unions of Borel sets with closed sections: Starting with a Borel set which is not such a union, Saint Raymond uses Hurewicz's construction to find a section for which Hurewicz's characterization of $\Sigma_{2}^{0}$ sets fails. Saint Raymond's result was extended by Bourgain to $\Sigma_{3}^{0}$ sets [B 1, B 2] using a somewhat related technique, and by Louveau [Lo 1, Lo 2] to all $\Sigma_{\xi}^{0}$ sets, by a quite different "Baire category" argument which relies heavily on tools from effective descriptive set theory. The results in $\$ 4$ allow us to give an alternative purely "classical" proof of Louveau's results, much in the spirit of Saint Raymond's proof for $\xi=2$.

As the proofs are more or less straightforward, we shall only indicate the main steps. If $E, F$ are two Polish spaces, and $A \subseteq E \times F, x \in E$, we denote by $A_{x}$ the section of $A$ at $x, A_{x}=\{y \in F \mid(x, y) \in A\}$.

Theorem 1 (Saint Raymond; Bourgain; Louveau). Let $E, F$ be Polish spaces, $\xi \geqslant 2$ some countable ordinal, $A^{0}, A^{1}$ two analytic subsets of $E \times F$.

(i) The set $\left\{x \in E \mid \Sigma_{\xi}^{0}\right.$ separates $\left.\left(A_{x}^{0}, A_{x}^{1}\right)\right\}$ is coanalytic in $E$.

(ii) If for all $x \in E \Sigma_{\xi}^{0}$ separates $\left(A_{x}^{0}, A_{x}^{1}\right)$, there exists a sequence $\left(B_{n}\right)_{n \in \omega}$ of Borel subsets of $E \times F$ such that $\cup_{n} B_{n}$ separates $A^{0}$ from $A^{1}$, and for each $n, B_{n}$ has sections in $\Pi_{\xi_{n}}^{0}$ for some $\xi_{n}<\xi$.

In particular, if $B \subset E \times F$ is Borel, then

(i) $\left\{x \in E \mid B_{x} \in \Sigma_{\xi}^{0}\right\}$ and $\left\{x \in E \mid B_{x} \in \Pi_{\xi}^{0}\right\}$ are coanalytic subsets of $E$.

(ii)' If $B$ has $\Sigma_{\xi}^{0}$ sections, then $B=\bigcup_{n} B_{n}$ where the $B_{n}$ 's are Borel sets with sections in $\Pi_{\xi_{n}}^{0}, \xi_{n}<\xi$. 
The main tool for getting this result from the results of $\S 4$ is a very general fact about closed games (it is an easy consequence of the "strategic basis theorem for $\Sigma_{1}^{0}$ games" of effective descriptive set theory, see [Mo] or [M-K], but can also be proven directly, using Kuratowski's second separation theorem).

Theorem. Let $E$ be some Polish space, and $J$ some Borel subset of $E \times 2^{\omega} \times \omega^{\omega}$ with closed sections. Viewing, for $x \in E, J_{x}$ as a closed (for II) game on $2 \times \omega$, assume player I has for all $x \in E$ a winning strategy in $J_{x}$. Then there exists a Borel map $\sigma: E \rightarrow \Sigma$ such that for all $x \in E, \sigma(x)$ is a winning strategy for player I in $J_{x}$.

We shall also need a refinement in the computation of the complexity of the filling-in functions $f^{\eta}$ and $F^{\eta}$ corresponding to the ramifications $\underline{R}^{\eta}$ built up in Theorem 3.2.

Lemma 2. Let $\eta$ be countable, $\underline{R}^{\eta}$ the ramification of Theorem 3.2. For sequences $u \in \omega^{k}$ and $v \in\left(\omega^{<\omega}\right)^{k+1}$, define

$$
\begin{aligned}
& C_{u, v}^{\eta}=\left\{(J, \sigma) \in \mathscr{J} \times \Sigma: u \text { is }\left(J, F^{\eta}(J, \sigma)\right)\right. \text {-legal and } \\
& \quad \text { for all } i \leqslant \operatorname{lh} u v(i)=f^{\eta}\left(J, \sigma, u \uparrow_{i}\right) \text { and } \\
&\left.F^{\eta}\left(J, \sigma, u \uparrow_{i}\right)=0 \text { for } i<\operatorname{lh} u \text { and } F^{\eta}(J, \sigma, u)=1\right\}
\end{aligned}
$$

Then for some ordinal $\xi(u, v)<1+\eta$, the set $C_{u, v}^{\eta}$ is $\Pi_{\xi(u, v)}^{0}$.

Proof. We know that $f^{\eta}$ and $F^{\eta}$ are class $\eta$ functions, hence certainly $C_{u, v}^{\eta}$ is $\Delta_{1+\eta}^{0}$. So we have to replace $\Delta_{1+\eta}^{0}$ by $\Pi_{\xi}^{0}$ for some $\xi<1+\eta$. We prove it by induction on $\eta$. Using the way $\underline{R}^{\eta}$ is defined at limit stages, the limit case is trivial. And the successor case $\eta^{\prime}=\eta+1$ easily reduces to the case of the basic ramification $\underline{R}$, by noticing that as $\underline{R}^{\eta+1}=\underline{R} \circ \underline{R}^{\eta}$,

$$
\begin{gathered}
(J, \sigma) \in C_{u, v}^{\eta+1} \leftrightarrow \exists u^{\prime} \in\left(\omega^{<\omega}\right)^{k+1}\left[\forall i \leqslant k f^{\eta}\left(R(J), \sigma, \bar{u}_{i}^{\prime}\right)=\bar{v}_{i}\right. \\
\left.\quad \text { and }\left(J, F^{\eta}(R(J), \sigma)\right) \in C_{u, u^{\prime}}^{1}\right]
\end{gathered}
$$

where $\bar{u}_{i}^{\prime}=u^{\prime}(0)^{\wedge} u(0)^{\wedge} \ldots \wedge u^{\prime}(i-1)$ and $\bar{v}_{i}=v(0)^{\wedge} u(0)^{\wedge} \ldots \wedge v(i-1)$. Now note that any such $u^{\prime}$ is a subsequence of $v$, hence the quantifier $\exists u^{\prime}$ is bounded. The first clause inside the brackets is $\Delta_{1+\eta}^{0}$ as $f^{\eta}$ is of class $\eta$, and the second clause is $\Pi_{1+\eta}^{0}$ as $F^{\eta}$ is of class $\eta$ too, once we know $C_{u, u^{\prime}}^{1}$ is $\Pi_{1}^{0}$.

To compute $C_{u, v}^{1}$, one has to go back to the definition of the filling-in function $f$ for the basic ramification (Lemma 3.6). The point is that as long as the answer by $F(J, \sigma)$ to $u \uparrow_{i}$ is 0 , one just has to verify that the corresponding $\bar{v}_{i}$ is the correct one - and this needs finite information on $J$ and $\sigma$, hence is $\Delta_{1}^{0}$. And for $u$ itself, one has to check all $(R(J), \sigma)$-legal extensions of $\bar{v}$ satisfy $\Delta_{1}^{0}$ clauses. This easily gives a $\Pi_{1}^{0}$ definition of $C_{u, v}^{1}$.

PROOF OF THEOREM 1 . The second part easily follows from the first. And applying the transfer Lemma 4.2 we may assume $F=2^{\omega}$, and (by using some Borel embedding if necessary) that $E=2^{\omega}$ too. Write $A^{0}$ and $A^{1}$ as projections of trees $T^{0}$ and $T^{1}$ on $2 \times 2 \times \omega$, and consider, for $x \in 2^{\omega}, J_{x}=J\left(A_{x}^{0}, A_{x}^{1}\right)$. The function $x \mapsto J_{x}$ is Borel, and by Theorem 1.1 
$\Sigma_{\xi}^{0}$ separates $\left(A_{x}^{0}, A_{x}^{1}\right) \leftrightarrow$ II has no winning strategy in $J_{x}$. This is easily computed as a $\Pi_{1}^{1}$ relation, and (i) is proved. Suppose now that for all $x \in 2^{\omega} \Sigma_{\xi}^{0}$ separates $\left(A_{x}^{0}, A_{x}^{1}\right)$. Then for all $x$, I wins $J_{x}$. Using the above theorem, a winning strategy $\sigma_{x}$ for I in $J_{x}$ can be found in a Borel way in $x$. Then let $\xi=1+\eta$, consider the sets $C_{u, v}^{\eta}$ of Lemma 2, and define, for $u \in \omega^{k}, v \in\left(\omega^{<\omega}\right)^{k}$

$$
B_{u, v}=\left\{(x, \alpha) \in 2^{\omega} \times 2^{\omega}:\left(J_{1}\left(A_{x}^{0}, A_{x}^{1}\right) \uparrow_{\alpha}, \sigma_{x} \uparrow_{\alpha}\right) \in C_{u, v}\right\}
$$

By the computations of Lemma 2, $B_{u, v}$ is Borel with $\Pi_{\xi(u, v)}^{0}$ sections. And by (the proof of) Theorem 1.1, the set $B=\bigcup_{u, v} B_{u, v}$ separates $A^{0}$ from $A^{1}$.

A very similar argument, using the other part of Theorem 1.1, yields an analogous result for the classes $D_{\eta}\left(\Sigma_{\xi}^{0}\right)$, thus reproving results of Debs [D] for $D_{2}\left(\Sigma_{2}^{0}\right)$ sets, and part of results in Louveau [Lo 4] (where Borel sets with sections of arbitrary given Wadge class are studied).

6.2. Effective results. We refer the reader to Moschovakis' book [Mo] and to the nice introductory paper of Kechris and Martin [M-K] for the basic notions of effective descriptive set theory.

We will not say much about the effective versions of the results in $\S 6.1$. They are obtained in a straightforward way by standard methods. The only point is that for recursive ordinal $\xi$, the fundamental sequences at the limit stages in the construction of the ramifications can be chosen in a $\Delta_{1}^{1}$ way, so that $H_{\xi}$ is $\Pi_{\xi}^{0}\left(\Delta_{1}^{1}\right)$, the functions $\rho^{\eta}$ and $f^{\eta}$ are $\Sigma_{1+\eta}^{0}\left(\Delta_{1}^{1}\right)$-recursive, and for $A_{0}, A_{1}$ in $\Sigma_{1}^{1}$, the game $J_{\xi}\left(A_{0}, A_{1}\right)$ is $\Pi_{1}^{0}\left(\Delta_{1}^{1}\right)$ for player II. Moreover, one easily checks that from the proof in [SR 3], the construction of the first class section $s$ of Saint Raymond's theorem is effective too, and gives a $\Sigma_{2}^{0}\left(\Delta_{1}^{1}\right)$-recursive function (provided $E, F$ are recursively presented, $\pi$ is $\Sigma_{1}^{0}\left(\Delta_{1}^{1}\right)$-recursive and $f$ is $\Sigma_{2}^{0}\left(\Delta_{1}^{1}\right)$-recursive). Altogether, the strategic basis theorem for $\Sigma_{1}^{0}$ games can then be used to give an alternative (and quite different) proof of the results of Louveau [Lo 2].

Once one has a closed game at one's disposal, another well-known technique allows to prove the existence of "largest $\Pi_{1}^{1}$ sets": E.g., one can prove, for $A \subset 2^{\omega}$ a $\Sigma_{1}^{1}$ set and $\xi$ some recursive ordinal, the existence of a largest $\Pi_{1}^{1}$ set $C$ disjoint from $A$ and such that the pair $(C, A)$ does not reduce $\Pi_{\xi}^{0}$, namely $\left\{\alpha \in 2^{\omega} \mid \exists \beta \in L_{\omega_{1}^{\alpha}}\right.$ ( $\beta$ codes a $\Pi_{<\xi}^{0}$ set $B$ with $\alpha \in B$ and $A \cap B=\varnothing$ ) .

Finally, our games also give sharp bounds on the complexity of winning strategies in Wadge games $G_{w}(A, B)$ for Borel $A, B$. Notice first that we cannot hope for $\Delta_{1}^{1}$ winning strategies: If say $A=\{\underline{0}\}$ and $B$ is some $\Pi_{2}^{0}$ subset of $2^{\omega}$ with no $\Delta_{1}^{1}$ member, then clearly II wins $G_{w}(A, B)$, but has no $\Delta_{1}^{1}$ winning strategy.

So the next result is best possible.

Proposition 1. Let $D \subset 2^{\omega}$ be a basis for $\Sigma_{1}^{1}$ sets closed under $\Delta_{1}^{1}$-reducibility. Suppose $\xi$ is a recursive ordinal, $A$ is $\Delta_{1}^{1}$ and $\Pi_{\xi}^{0} \backslash \Sigma_{\xi}^{0}$, and $\left(A_{0}, A_{1}\right)$ is a pair of disjoint $\Sigma_{1}^{1}$ subsets of $2^{\omega}$. In Wadge's extended game $G_{\omega}^{*}\left(A ; A_{0}, A_{1}\right)$, one of the players has a winning strategy in $D$.

PRoof. Using the remarks above, we may assume $A$ is $\Pi_{\xi}^{0}\left(\Delta_{1}^{1}\right)$. Winning strategies for II in games $J_{\xi}\left(A_{0}, A_{1}\right)$ for $\Sigma_{1}^{1}$ sets $A_{0}, A_{1}$ can clearly be found in $D$, as $J_{\xi}$ is $\Pi_{1}^{0}$ for II, hence $\left\{\sigma\right.$ : $\sigma$ is winning for II in $\left.J_{\xi}\left(A_{0}, A_{1}\right)\right\}$ is $\Sigma_{1}^{1}$. Coming back to the proof 
of Corollary 1.2. (and using the fact that if $\left(A_{0}, A_{1}\right)$ is $\Sigma_{\xi}^{0}$-separable, a separating set $C$ can be found in $\Sigma_{\xi}^{0}\left(\Delta_{1}^{1}\right)$, again by the discussion above), one easily checks that the proof of the proposition easily reduces to the fact that for $\xi$ recursive and $A \in \Pi_{\xi}^{0}$, player II wins the Wadge game $G_{w}\left(A, H_{\xi}\right)$ via some $\Delta_{1}^{1}$ winning strategy. But this is easily proved - by looking at the proof that $H_{\xi}$ is strategically complete $\Pi_{\xi}^{0}$.

7. Extensions to higher levels in the projective hierarchy. We again refer the reader to Moschovakis' book [Mo], especially Chapters 6 and 8, for the relevant notions of descriptive set theory and set theory we use in this section.

7.1. The $\Sigma_{2 n+1}^{1}$ case, $n \geqslant 1$. Assuming all $\Delta_{2 n}^{1}$ games are determined, the structure theory of the projective classes $\Sigma_{2 n+1}^{1}, n \geqslant 1$, looks very much like the basic structure theory of $\Sigma_{1}^{1}$ sets. In particular, Moschovakis's third periodicity theorem [Mo, 6E1] allows to compute the complexity of winning strategies for player I in $\Pi_{2 n}^{1}$ games (for II). Using this fundamental tool, and the ramifications, we can prove for this case the following result, which extends work of Kechris [Ke] who proved (b) for $n=1$, and Louveau [Lo 3] who proved (c) by a different method.

Let us fix some ordinal $\xi<\omega_{1}^{C K}$, and denote by $\Sigma_{\xi}^{0}\left(\Delta_{2 n+1}^{1}\right)$ the class of all $\Sigma_{\xi}^{0}$ subsets of $2^{\omega}$ which do admit a $\Delta_{2 n+1}^{1}$ code (in some canonical coding of $\Sigma_{\xi^{-}}^{0}$ constructions by elements of $\omega^{\omega}$; this notion does not depend on the particular - reasonable-coding which is chosen).

THEOREM 1. Assume all $\Delta_{2 n}^{1}$ games are determined, and let $\xi<\omega_{1}^{C K}$.

(a) If $\left(A_{0}, A_{1}\right)$ is a pair of disjoint $\Sigma_{2 n+1}^{1}$ sets in $2^{\omega}$ (or more generally in some recursively presented Polish space $E$ ), then either $\left(A_{0}, A_{1}\right)$ reduces the class $\Pi_{\xi}^{0}$ (in a one-to-one way for $\xi \geqslant 3)$, or $\Sigma_{\xi}^{0}\left(\Delta_{2 n+1}^{1}\right)$ separates $\left(A_{0}, A_{1}\right)$.

(b) In particular if $A_{0}, A_{1}$ are $\Sigma_{2 n+1}^{1}$ and $\Sigma_{\xi}^{0}$ separates $\left(A_{0}, A_{1}\right)$, then $\Sigma_{\xi}^{0}\left(\Delta_{2 n+1}^{1}\right)$ also separates $\left(A_{0}, A_{1}\right)$.

(c) $\Sigma_{\xi}^{0}\left(\Delta_{2 n+1}^{1}\right)=\Delta_{2 n+1}^{1} \cap \Sigma_{\xi}^{0}$, i.e. any $\Sigma_{\xi}^{0}$ set in $\Delta_{2 n+1}^{1}$ admits a $\Delta_{2 n+1}^{1} \Sigma_{\xi^{-}}^{0}$ construction.

Proof. We just outline the proof, and indicate how to modify the techniques of $\S \S 2$ and 3 to get the result. We fix once and for all two $\Pi_{2 n}^{1}$ sets $P_{0}$ and $P_{1}$ in $2^{\omega} \times \omega^{\omega}$ such that

$$
A_{i}=p\left(P_{i}\right)=\left\{\alpha \in 2^{\omega} \mid \exists \beta \in \omega^{\omega}(\alpha, \beta) \in P_{i}\right\} .
$$

We first modify the game $J_{1}$ as follows: In $J_{1}^{*}\left(A_{0}, A_{1}\right)$, player I produces $\varepsilon \in 2^{\omega}$, player II produces $(\alpha, \beta) \in 2^{\omega} \times \omega^{\omega}$ as before. The play $(\varepsilon, \alpha, \beta)$ is a win for II if $\left(\alpha, \beta^{*}\right) \in P_{i}$, where we set $i=0$ and $\beta^{*}=\beta$ if $\varepsilon=\underline{0}$, and if $\varepsilon \neq \underline{0}$ and $k$ is least so that $\varepsilon(k)=1$, we set $i=1$ and $\beta^{*}(j)=\beta(k+j)$. This game is clearly $\Pi_{2 n}^{1}$ (for II), and arguments similar to those for $J_{1}$ show that

(i) If $(\varepsilon, \alpha, \beta)$ is a win for II, then $\varepsilon=\underline{0}$ implies $\alpha \in A_{0}$ and $\varepsilon \neq \underline{0}$ implies $\alpha \in A_{1}$.

(ii) If we define an open set $C^{*} \subset 2^{\omega} \times \Sigma$ by

$$
C^{*}=\left\{(\alpha, \sigma) \mid \exists u \in \omega^{<\omega}\left(\sigma\left(\alpha \uparrow_{\ln u}, u\right)=1\right)\right\}
$$

and if $\sigma$ is a winning strategy for player I in some game $J_{1}{ }^{*} \uparrow_{\alpha}$, for $\alpha \in 2^{\omega}$, then $\alpha \in A_{0}$ implies $(\alpha, \sigma) \in C^{*}$ and $\alpha \in A_{1}$ implies $(\alpha, \sigma) \notin C^{*}$. 
Next we have to define corresponding modifications $J_{\xi}^{*}$ of the games $J_{\xi}$. For this, we make the ramification $\underline{R}^{\eta}$, with $\xi=1+\eta$, act on $\Pi_{2 n}^{1}$ games. And as the notion of legal finite position is no more meaningful, we use the projection function $\rho^{\eta}$ instead of $r^{\eta}$ : In $J_{\xi}^{*}\left(A_{0}, A_{1}\right)$, I plays $\varepsilon \in 2^{\omega}$, II plays $(\alpha, \beta) \in 2^{\omega} \times \omega^{\omega}$, and II wins if the play $\left\langle\rho_{0}^{\eta}(\varepsilon), \alpha, \beta \circ \rho_{1}^{\eta}(\varepsilon)\right\rangle$ is a win for II in $J_{1}^{*}\left(A_{0}, A_{1}\right)$. Using the properties of $\rho^{\eta}$ and fact (i) above, one easily checks that a winning strategy for II in $J_{\xi}^{*}$ gives a winning strategy for II in $G_{w}^{*}\left(H_{\xi} ; A_{0}, A_{1}\right)$, hence $\left(A_{0}, A_{1}\right)$ reduces $\Pi_{\xi}^{0}$.

Suppose now $\sigma$ is a winning strategy for I in $J_{\xi}^{*}\left(A_{0}, A_{1}\right)$, and let $\sigma \uparrow_{\alpha}$ be the corresponding strategy in $J_{\xi}^{*}\left(A_{0}, A_{1}\right) \uparrow_{\alpha}$. We define a class $\eta$ function $F_{\eta}^{*}: \Sigma \rightarrow \Sigma$ by $F_{\eta}^{*}(\sigma)=F^{\eta}\left(\omega^{<\omega}, \sigma\right)$, i.e. $F_{\eta}^{*}$ corresponds to the filling-in function associated with the trivial game $J=\omega^{<\omega}$ (again we are forgetting about the legality of finite positions). Note that in this case $R^{\eta}(J)=\omega^{<\omega}$ too. Moreover one easily verifies by induction the following commutativity of $\rho^{\eta}$ and $F_{\eta}^{*}$ : For any $\beta \in \omega^{\omega}$ and $\sigma \in \Sigma$, if $\bar{f}_{\eta}^{*}(\beta)$ is the play obtained by using the filling-in function (for $J=\omega^{<\omega}$ ), and $\varepsilon=\bar{\sigma}\left(\bar{f}_{\eta}^{*}(\beta)\right)$ the answer by $\sigma$, then $\bar{f}_{\eta}^{*}(\beta) \circ \rho_{1}^{\eta}(\varepsilon)=\beta$ and $\rho_{0}^{\eta}(\varepsilon)$ is the corresponding answer to $\beta$ by $F_{\eta}^{*}(\sigma)$.

From this, it easily follows that applying $F_{\eta}^{*}$ to $\sigma \uparrow_{\alpha}$ gives a winning strategy for Player I in $J_{1}^{*}\left(A_{0}, A_{1}\right) \uparrow_{\alpha}$, and the set $C_{\sigma}^{*}=\left\{\alpha \in 2^{\omega} \mid\left(\alpha, F_{\eta}^{*}\left(\sigma \uparrow_{\alpha}\right)\right) \in C^{*}\right\}$ is $\Sigma_{\xi}^{0}$ and separates $A_{0}$ from $A_{1}$. Finally by choosing the strategy $\sigma$ in $\Delta_{2 n+1}^{1}$, using Moschovakis' strategic basis theorem, one easily checks that $C_{\sigma}^{*}$ is in $\sum_{\xi}^{0}\left(\Delta_{2 n+1}^{1}\right)$.

7.2. The $\Sigma_{2}^{1}$ case. In this subsection, we show how to modify the use of ramifications to study separation of $\kappa$-Suslin sets by Borel sets, and in particular give an alternative proof of a result of Stern [St] about separation of $\Sigma_{2}^{1}$ sets.

Let $\kappa$ be some infinite cardinal. A set $A \subseteq 2^{\omega}$ is $\kappa$-Suslin if for some tree $T$ on $2 \times \kappa, A=p([T])=\left\{\alpha \in 2^{\omega} \mid \exists f \in \kappa^{\omega} \forall n\left(\alpha \uparrow_{n}, f \uparrow_{n}\right) \in T\right\}$ (so that $\Sigma_{1}^{1}$ sets correspond to $\aleph_{0}$-Suslin). We define the family of $\kappa$-Borel sets as the least family of subsets of $2^{\omega}$ containing the basic open sets and closed under unions and intersections of at most $\kappa$-sequences. This family admits a natural ranking, and a natural notion of coding: We let $D_{0}^{\kappa}=\left\{0^{\wedge} s: s \in 2^{<\omega}\right\}$; for $0^{\wedge} s \in D_{0}^{\kappa}$, its coded set is $\check{N}_{s}=\left\{\alpha \in 2^{\omega} \mid \alpha \uparrow_{\mathrm{lh} s} \neq s\right\}$, and $\Sigma_{0}^{\kappa}=\left\{\check{N}_{s} \mid 0^{\wedge} s \in D_{0}^{\kappa}\right\}$. Now by induction, we set $D_{\xi}^{\kappa}=\left\{1^{\wedge} f \mid f: \kappa \rightarrow \bigcup_{\xi^{\prime}<\xi} D_{\xi^{\prime}}^{\eta}\right\}$; the set coded by $1^{\wedge} f \in D_{\xi}$ is $A_{1^{\wedge} f}=\bigcup_{\xi<\kappa}\left(\check{A}_{f(\xi)}\right)$; and $\sum_{\xi}^{\kappa}=\left\{A_{1^{\wedge} f} \mid 1^{\wedge} f \in D_{\xi}^{\kappa}\right\}$ is the set of $\kappa$-unions of complements of sets in $\bigcup_{\xi^{\prime}<\xi} \sum_{\xi^{\prime}}^{\kappa}$

If now $M$ is some inner model, we say that $A$ is $\kappa$-Suslin over $M$ if some tree $T$ on $2 \times \kappa$ so that $A=p([T])$ can be found in $M$, and we say $A \in \Sigma_{\xi}^{\kappa}(M)$ if some code $f \in D_{\xi}^{\kappa} \cap M$ with $A=A_{f}$ does exist.

THEOREM 2. Let $M$ be some inner model, $\xi$ an ordinal which is countable in $M$, and $\kappa$ an infinite cardinal. If $\left(A_{0}, A_{1}\right)$ is a pair of disjoint $\kappa$-Suslin over $M$ subsets of $2^{\omega}$, then either

(i) the pair $\left(A_{0}, A_{1}\right)$ reduces $\Pi_{\xi}^{0}$, or

(ii) there is a set $C \in \Sigma_{\xi}^{\kappa}(M)$ which separates $A_{0}$ from $A_{1}$ ((i) and (ii) are not exclusive). 
Corollary 3 (STERn [St]). Let $\xi$ be some ordinal $<\aleph_{1}^{L}$, and assume $\boldsymbol{\aleph}_{\xi}^{L}$ is countable. Let $A_{0}, A_{1}$ be two disjoint $\Sigma_{2}^{1}$ subsets of $2^{\omega}$. If $\Sigma_{\xi}^{0}$ separates the pair $\left(A_{0}, A_{1}\right)$, and $M$ is any inner model where $\aleph_{\xi}^{L}$ is countable, a code for a $\Sigma_{\xi}^{0}$ set separating $A_{0}$ from $A_{1}$ can be found in $M$.

Proof of Corollary 3. The sets $A_{0}, A_{1}$ are $\aleph_{1}$-Suslin over $L$, so that we can apply Theorem 2 and by the hypothesis get a $\Sigma_{\xi}^{\aleph_{1}}(L)$ set $A$ separating $A_{0}$ from $A_{1}$. We now claim that for $\eta<\xi$

$$
\operatorname{card}\left(\Sigma_{\eta}^{\aleph_{1}}(L)\right) \leqslant \operatorname{card}\left(\aleph_{\eta+1}^{L}\right) .
$$

Granting this, a $\Sigma_{\xi}^{\aleph_{1}}$-code for $A$ can easily be transformed in $M$ in a code for $A$ as a $\Sigma_{\xi}^{0}$-set.

So it remains to prove the claim. But note that for each $\eta$-code $1^{\wedge} f$, with $1 \leqslant \eta<\xi$, the coded set $A_{1^{\wedge} f}$ depends only on the range $f^{\prime \prime}\left(\aleph_{1}\right)$ of $f$. So if we define a function $\varphi$ by induction by $\varphi\left(1^{\wedge} f\right)=\left\{\varphi(f(\theta)): \theta<\aleph_{1}\right\}$, we get for $\eta<\xi$

$$
\operatorname{card}\left(\Sigma_{\eta}^{\aleph_{1}}(L)\right) \leqslant \operatorname{card}\left(\varphi^{\prime \prime}\left(D_{\eta}^{\aleph_{1}} \cap L\right)\right) .
$$

And by an immediate computation in $L, \operatorname{card}\left(\varphi^{\prime \prime}\left(D_{\eta}^{\aleph_{1}} \cap L\right)\right) \leqslant \operatorname{card} \aleph_{\eta+1}^{L}$ so we are done.

The proof of Theorem 2 is totally parallel to the proof of Theorem 1.1. Again we just outline the minor modifications which are needed for it. We fix once and for all $A_{0}, A_{1}$ and trees $T_{0}, T_{1}$ in $M$ on $2 \times \kappa$ with $A_{i}=p\left(\left[T_{i}\right]\right)$.

We now define a game $J_{1}^{\kappa}\left(A_{0}, A_{1}\right)$ : I plays $\varepsilon \in 2^{\omega}$, II plays $\alpha \in 2^{\omega}$ and $f \in \kappa^{\omega}$. II wins if all finite positions $\left(\varepsilon \uparrow_{k}, \alpha \uparrow_{k}, f \uparrow_{k}\right)$ are legal, where legality is defined by the following: If $\varepsilon(i)=0$ for all $i<k,\left(\alpha \uparrow_{k}, f \uparrow_{k}\right)$ must be in $T_{0}$. And if $i_{0}$ is least with $\varepsilon\left(i_{0}\right)=1,\left(\alpha \uparrow_{k-i_{0}},\left\langle f\left(i_{0}\right), f\left(i_{0}+1\right), \ldots, f(k-1)\right\rangle\right) \in T_{1}$. One easily checks that if $(\varepsilon, \alpha, f)$ is a win for II in $J_{1}^{\kappa}\left(A_{0}, A_{1}\right)$, then $\varepsilon=\underline{0}$ implies $\alpha \in A_{0}$ and $\varepsilon \neq \underline{0}$ implies $\alpha \in A_{1}$.

If we now set $\mathscr{J}_{\kappa}=\{J: J$ is a tree on $2 \times \kappa\}$, with $\Sigma_{\kappa}=\left\{\sigma: \kappa^{<\omega} \rightarrow 2\right\}$ the corresponding set of strategies, and if we let $C^{\kappa} \subset 2^{\omega} \times \Sigma_{\kappa}$ be defined by $(\alpha, \sigma) \in$ $C^{\kappa} \leftrightarrow \exists u \in \kappa^{<\omega}\left(u\right.$ is $\sigma$-legal in $J_{1}^{\kappa}\left(A_{0}, A_{1}\right) \uparrow_{\alpha}$ and $\left.\sigma(u)=1\right)$, one checks that for $\sigma$ a winning strategy for $\mathrm{I}$ in $J_{1}^{\kappa}\left(A_{0}, A_{1}\right) \uparrow_{\alpha}$, one has $\alpha \in A_{0}$ implies $(\alpha, \sigma) \in C^{\kappa}$ and $\alpha \in A_{1}$ implies $(\alpha, \sigma) \notin C^{\kappa}$.

Now as $\xi$ is a countable ordinal in $M$, the construction of the ramification function $r^{\eta}$, for $\xi=1+\eta$, can be done inside $M$, and the function $r^{\eta}$ also acts on closed games on $2 \times \kappa$, by defining $R_{\kappa}^{\eta}: \mathscr{J}_{\kappa} \rightarrow \mathscr{J}_{\kappa}$, for $J$ a tree on $2 \times \kappa$, by

$$
R_{\kappa}^{\eta}(J)=\left\{(u, v) \in(2 \times \kappa)^{<\omega}: \forall i \leqslant \operatorname{lh} u r^{\eta}\left(u \uparrow_{i}, v \uparrow_{i}\right) \in J\right\},
$$

where $r^{\eta}(u, v)=\left\langle r_{0}^{\eta}(u), v \circ r_{1}^{\eta}(u)\right\rangle$.

From this, one can define a closed game $J_{\xi}^{\kappa}\left(A_{0}, A_{1}\right)$ on $2 \times 2 \times \kappa$ by the following: I plays $\varepsilon \in 2^{\omega}$, II plays $(\alpha, f) \in 2^{\omega} \times \kappa^{\omega}$, and II wins if $(\varepsilon, f)$ is a win in the game $R_{\kappa}^{\eta}\left(J_{1}^{\kappa}\left(A_{0}, A_{1}\right) \uparrow_{\alpha}\right)$. This game is clearly closed (hence determined), and its associated tree on $2 \times 2 \times \kappa$ is in $M$, so that by absoluteness winning strategies can be found in $M$. The case where II has a winning strategy creates no difficulty: One gets a corresponding winning strategy in $G_{w}^{*}\left(H_{\xi} ; A_{0}, A_{1}\right)$, and the pair $\left(A_{0}, A_{1}\right)$ reduces $\Pi_{\xi}^{0}$. 
Now if player I has some winning strategy $\sigma$ in $M$ in the game $J_{\xi}^{\kappa}\left(A_{0}, A_{1}\right)$, one can imitate the proof of the existence of the filling-in functions $f^{\eta}$ and $F^{\eta}$ to get a definable in $M$ function $F_{\kappa}^{\eta}: \mathscr{J}_{\kappa} \times \Sigma_{\kappa} \rightarrow \Sigma_{\kappa}$ such that for all $\alpha \in 2^{\omega}$ the function $\sigma_{\alpha}^{*}=F_{\kappa}^{\eta}\left(J_{1}^{\kappa}\left(A_{0}, A_{1}\right) \uparrow_{\alpha}, \sigma \uparrow_{\alpha}\right)$ is winning for player I in $J_{1}^{\kappa}\left(A_{0}, A_{1}\right) \uparrow_{\alpha}$, so that the set $C_{\sigma}^{\kappa}=\left\{\alpha \in 2^{\omega} \mid\left(\alpha, \sigma_{\alpha}^{*}\right) \in C^{\kappa}\right\}$ separates $A_{0}$ from $A_{1}$. And $C_{\sigma}^{\kappa}$ can be computed as a $\sum_{\xi}^{\kappa}(M)$-set. We leave the straightforward but tedious details to the reader.

Notice that in case II has a winning strategy in $J_{\xi}^{\kappa}\left(A_{0}, A_{1}\right)$, only countably many ordinals $<\kappa$ are in the range of the strategy. In particular if $A_{0}, A_{1}$ are $\Sigma_{2}^{1}$ sets with trees $T_{0}, T_{1}$ on $2 \times \aleph_{1}$, and if we define the usual $\Sigma_{1}^{1}$ approximations $\left(A_{i}^{\theta}\right)_{\theta<\aleph_{1}}$ by $A_{i}^{\theta}=\left\{\alpha \in 2^{\omega}: \exists f \in \theta^{\omega} \forall n\left(\alpha \uparrow_{n}, f \uparrow_{n}\right) \in T_{i}\right\}$, we get in case (i) that for some $\theta<\boldsymbol{\aleph}_{1}$, the pair $\left(A_{0}^{\theta}, A_{1}^{\theta}\right)$ already reduces $\Pi_{\xi}^{0}$. This was also obtained in [St], and Stern used it to prove that if $\aleph_{\xi}^{L}$ is countable, the relation $\Sigma_{\xi}^{0}$ separates $\left(A_{0}, A_{1}\right)$, for $A_{0}, A_{1}$ ranging over $\Sigma_{2}^{1}$ sets, is a $\Pi_{2}^{1}$ relation.

\section{REFERENCES}

[B 1] J. Bourgain, $F_{\sigma \delta}$ sections of Borel sets, Fund. Math. 107 (1980), 129-133.

[B 2] _ Borel sets with $F_{\text {o } \delta}$ sections, Fund. Math. 107 (1980), 149-159.

[Da] M. Davis, Infinite games of perfect information, Ann. of Math. Studies, no. 52, Princeton Univ. Press, Princeton, N.J., 1964, pp. 445-448.

[D] G. Debs, Un résultat d'uniformisation borélienne, Proc. Amer. Math. Soc. 92 (1984), 445-448.

[De] C. Dellacherie, Ensembles analytiques: Théorèmes de séparation et applications, Sém. Probabilités Strasbourg IX, (1973-74), Lecture Notes in Math., vol. 465, Springer-Verlag, Berlin and New York, 1975.

[F] H. Friedman, Higher set theory and mathematical practice. Ann. of Math. Logic 2 (1971), 326-357.

[Ha] L. Harrington, Analytic determinacy and $0^{\#}$, J. Symbolic Logic 43 (1978), 685-693.

[Hu] W. Hurewicz, Relativ perfekte Teile von Punktmengen und Mengen (A), Fund. Math. 12 (1928), 78-109.

[J] T. John, Thesis, Berkeley, 1983.

[Ke] A. S. Kechris, A basis theorem for $\Delta_{3}^{1}$ Borel sets, circulated notes.

$[\mathbf{K}-\mathbf{M}] \mathrm{K}$. Kunen and A. W. Miller, Borel and projective sets from the point of view of compact sets, Math. Proc. Cambridge Philos. Soc. 94 (1983), 399-409.

[Kun] K. Kunugui, Contributions à la théorie des ensembles boréliens et analytiques III, J. Fac. Sci. Hokkaido Imp. Univ. 8 (1939-40), 79-108.

[Kur] K. Kuratowski, Topologie, vol. 1, PWN, Warszawa, 1958.

[L-SR] A. Louveau et J. Saint Raymond, Caractérisation par des jeux fermés de la classe de Baire des Boréliens, C. R. Acad. Sci. Paris 300 (1985).

[Lo 1] A. Louveau. Recursivity and compactness, Higher Set Theory, Proc. Oberwolfach 1977, (G. H. Müller and D. S. Scott, eds.), Lecture Notes in Math., vol. 669, Springer-Verlag, Berlin and New York, 1978, pp. 303-338.

[Lo 2] __ A separation theorem for $\Sigma_{1}^{1}$ sets, Trans. Amer. Math. Soc. 260 (1980), 363-378.

[Lo 3] _ Borel sets and the analytical hierarchy, Proc. Herbrand Sympos., Logic Coll. 81 (J. Stern, ed.), North-Holland, 1982, pp. 209-215.

[Lo 4] _ Some results in the Wadge Hierarchy of Borel sets, Cabal Sem. 79-81, (A. S. Kechris, D. A. Martin and Y. N. Moschovakis, eds.), Lecture Notes in Math., vol. 1019, Springer-Verlag, 1983, pp. 28-55.

[Lu] N. Luzin, Leçons sur les ensembles analytiques et leurs applications, 2nd ed., Chelsea, New York, 1972.

[Ma] D. A. Martin, Borel determinacy, Ann. of Math. (2) 102 (1975), 363-371.

[M-K] D. A. Martin and A. S. Kechris, Infinite games and effective descriptive set theory, Analytic Sets (C. A. Rogers et al.), Academic Press, New York, 1980.

[Mo] Y. N. Moschovakis, Descriptive set theory, North-Holland, 1980.

[N] P. S. Novikov, Sur une propriété des ensembles analytiques, Dokl. Akad. Nauk SSSR 3(5), (1934), 273-276. 
[SR 1] J. Saint Raymond, Boréliens à coupes $K_{\sigma}$, Bull. Soc. Math. France 104 (1976), 389-406.

[SR 2] La structure borélienne d'Effros est-elle standard?, Fund. Math. 100 (1978), 201-210.

[SR 3] Fonctions boréliennes sur un quotient, Bull. Sci. Math. 100 (1976), 141-147.

[S] J. R. Steel, Analytic sets and Borel isomorphisms, Fund. Math. 108 (1980), 83-88.

[St] J. Stern, On Luzin's restricted continuum problem Ann. of Math. (2) 120 (1984), 7-37.

[vE 1] F. van Engelen, Homogeneous Borel sets, Preprint.

[vE 2] Characterizations of the countable infinite product of rationals and related problems, Preprint.

[vE-vM] F. van Engelen and J. van Mill, Borel sets in compact spaces: Some Hurewicz-type theorems, Fund. Math. 124 (1984), 271-286.

[vW] R. van Wesep, Wadge degrees and descriptive set theory, Cabal Sem. 76-77, (A. S. Kechris and Y. N. Moschovakis, eds.), Lecture Notes in Math., vol. 689, Springer-Verlag, Berlin and New York, 1978, pp. $151-170$.

[W 1] W. W. Wadge, Degrees of complexity of subsets of the Baire space, Notices Amer. Math. Soc. (1972), A-714.

[W 2] _ Thesis, Berkeley, 1984.

Equipe d'Analyse, U.A. N 754 , Universite Paris VI, 4, Place Jussieu, Tour 46 / 0 - 4ime Etage, 75230 - PARIS CEDEX 05, FranCE 